\title{
GAUSSIAN AND NON-GAUSSIAN FLUCTUATIONS FOR MESOSCOPIC LINEAR STATISTICS IN DETERMINANTAL PROCESSES ${ }^{1}$
}

\author{
By KURT JOHANSSON AND GAULTIER LAMBERT
}

\author{
KTH Royal Institute of Technology and University of Zürich
}

\begin{abstract}
We study mesoscopic linear statistics for a class of determinantal point processes which interpolate between Poisson and random matrix statistics. These processes are obtained by modifying the spectrum of the correlation kernel of the Gaussian Unitary Ensemble (GUE) eigenvalue process. An example of such a system comes from considering the distribution of noncolliding Brownian motions in a cylindrical geometry, or a grand canonical ensemble of free fermions in a quadratic well at positive temperature. When the scale of the modification of the spectrum of the correlation kernel, related to the size of the cylinder or the temperature, is different from the scale in the mesoscopic linear statistic, we obtain a central limit theorem (CLT) of either Poisson or GUE type. On the other hand, in the critical regime where the scales are the same, we observe a non-Gaussian process in the limit. Its distribution is characterized by explicit but complicated formulae for the cumulants of smooth linear statistics. These results rely on an asymptotic sinekernel approximation of the GUE kernel which is valid at all mesoscopic scales, and a generalization of cumulant computations of Soshnikov for the sine process. Analogous determinantal processes on the circle are also considered with similar results.
\end{abstract}

\section{CONTENTS}

1. Introduction and results . . . . . . . . . . . . . . . . . . . . . . . . 1202

1.1. Introduction . . . . . . . . . . . . . . . . . . . . . . 1202

1.2. The modified Gaussian unitary ensembles . . . . . . . . . . . . . . . . . . 1203

1.3. Main results . . . . . . . . . . . . . . . . . . . . . . . . . . . . . 1208

1.4. Outline of the main ideas . . . . . . . . . . . . . . . . . . . . . . . . . 1213

1.5. Overview of the rest of the paper . . . . . . . . . . . . . . . . . . 1217

2. Modified circular unitary ensembles . . . . . . . . . . . . . . . . . . . . . . . 1218

2.1. Soshnikov's method: Proof of Lemma $1.14 \ldots \ldots$. . . . . . . . . . . . . . . . . . 1218

2.2. Central limit theorems . . . . . . . . . . . . . . . . . . . . . . . . . . . . . 1219

2.3. The critical regime . . . . . . . . . . . . . . . . . . . . . . . . . . 1222

3. Central limit theorems for the modified GUEs . . . . . . . . . . . . . . . . . . 12225

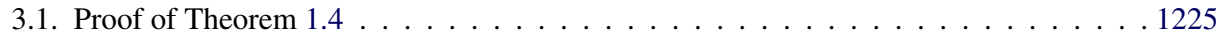

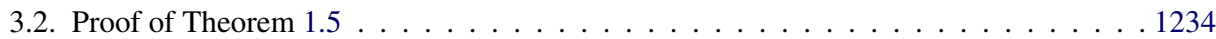

Received July 2015; revised October 2016.

${ }^{1}$ Supported by the Grant KAW 2010.0063 from the Knut and Alice Wallenberg Foundation.

MSC2010 subject classifications. 60B20, 60G55, 60F05.

Key words and phrases. Gaussian unitary ensemble, determinantal point processes, central limit theorem, cumulant method, transition. 
4. Cumulants of the critical models . . . . . . . . . . . . . . . . . . . 1235

4.1. Asymptotically equivalent kernels for the critical modified GUEs . . . . . . . . . . 1235

4.2. Proof of Theorem 1.7 . . . . . . . . . . . . . . . . . . . . . 1237

4.3. Properties of the random process $\Xi_{\Psi, \tau} \ldots \ldots$. . . . . . . . . . . . . . 1254

4.4. Fluctuations of the critical MNS ensemble . . . . . . . . . . . . . . . . . . 1259

Appendix A: Asymptotics of the Hermite polynomials . . . . . . . . . . . . . . . . 1263

Appendix B: Variance in the critical regime . . . . . . . . . . . . . . . . . . . . . . . . . . . . . . . . . . . . . . .

Appendix C: The third and fourth cumulants . . . . . . . . . . . . . . . . . . . . . . 1270

Acknowledgements . . . . . . . . . . . . . . . . . . . . . . . . 1276

References . . . . . . . . . . . . . . . . . . . . . 1276

\section{Introduction and results.}

1.1. Introduction. Recently, there has been a lot of discussion about universality of random matrix statistics at mesoscopic or intermediate scales. For instance, the proofs of the local semicircle law and the Wigner-Dyson-Gaudin-Mehta conjecture, see [5, 18] for further references, the work [16, 17] on random band matrices and the so-called metal-insulator transition, or the CLT for linear statistics of orthogonal polynomial ensembles [8]. One motivation to investigate these models comes from E. Wigner's fundamental observation that the spectral statistics of complicated quantum systems exhibit universal patterns. On the other hand, eigenvalues of quantum systems which are classically integrable are expected to be described by Poisson statistics [20]. Therefore, it is natural to investigate the transition from Poisson to random matrix statistics at intermediate scales. There are many ways to interpolate between the two point processes, for instance using Dyson's Brownian motion, one gets a determinantal process called the deformed Gaussian unitary ensemble [23]. For this model, the transition has been investigated using mesoscopic linear statistics in [14]. The authors proved different CLTs with fluctuations which depend on the scale of the test function that samples the eigenvalues. In this paper, we will study the fluctuations of another general class of determinantal processes which interpolate between Poisson and GUE statistics that we call modified GUEs (see Definition 1.1). Instead of adding an independent matrix to the GUE, we directly modify or mollify the spectrum of the correlation kernel of the process. This has the effect of introducing some extra disorder in the system while keeping the determinantal structure. Our main motivation to study such ensembles comes from the so-called MNS ensemble which was introduced by Moshe, Neuberger and Shapiro in [29] (see also [11, 23]), and corresponds to the joint probability density function of the positions of a grand canonical system of free fermions at positive temperature confined in a harmonic potential. In general, it is also of interest to investigate fluctuations of determinantal processes whose correlation kernels are not necessarily reproducing. 
1.2. The modified Gaussian unitary ensembles. Let $\mathfrak{X}$ be a Polish space equipped with some reference measure $d \mu$. Note that in the sequel, we will only be interested in the two cases $\mathfrak{X}=\mathbb{R}$ or the unit circle $\mathbb{T}$ equipped with the Lebesgue measure. A point process is a random measure on $\mathfrak{X}$ of the form $\Xi=\sum \delta_{X_{i}}$. The support of the measure $\Xi$ is the object of interest, it is called a point configuration $\left\{X_{i}\right\}$ and we assume that it has neither double point nor accumulation point. Point processes are usually described by their correlation functions $\rho_{1}, \rho_{2}, \ldots$ which are characterized by

$$
\mathbb{E}\left[\prod_{i} 1+g\left(X_{i}\right)\right]=\sum_{k=1}^{\infty} \frac{1}{k !} \int_{\mathfrak{X}^{k}} \prod_{i} g\left(x_{i}\right) \rho_{k}\left(x_{1}, \ldots, x_{k}\right) d \mu\left(x_{1}\right) \cdots d \mu\left(x_{k}\right)
$$

for any continuous function $g: \mathfrak{X} \rightarrow \mathbb{C}$ with compact support. A point process is called determinantal if all its correlation functions exist and satisfy the identity

$$
\rho_{k}\left(x_{1}, \ldots, x_{k}\right)=\operatorname{det}_{k \times k}\left[K\left(x_{i}, x_{j}\right)\right] .
$$

Hence, a determinantal process is characterized by its correlation kernel $K: \mathfrak{X} \times$ $\mathfrak{X} \rightarrow \mathbb{R}$ and we will denote by $\mathbb{E}_{K}$ the corresponding probability measure on the space of point configurations. Moreover, the kernel $K$ defines an integral operator $\mathcal{K}$ on $L^{2}(d \mu)$ which is locally trace-class so that the RHS of formula (1.1) is a Fredholm determinant:

$$
\mathbb{E}_{K}\left[\prod_{i} 1+g\left(X_{i}\right)\right]=\operatorname{det}[I+\mathcal{K} g]_{L^{2}(d \mu)} .
$$

In most cases, the operator $\mathcal{K}$ is self-adjoint (although there are natural examples of non-Hermitian determinant processes, such as the deformed GUE studied in [14]). Then the kernel $K$ defines a determinantal process if and only if all the spectrum of the operator $\mathcal{K}$ lies in $[0,1]$. These facts are well known and we refer to [21, $22,38]$ for different introductions to the theory of (determinantal) point processes and to the survey [4] for an overview of several applications. In this paper, we will investigate examples of determinantal processes with correlation kernels of the general form

$$
K(x, y)=\sum_{k=0}^{\infty} p_{k}^{N} \vartheta_{k}(x) \vartheta_{k}(y),
$$

where $\left(\vartheta_{k}\right)_{k=0}^{\infty}$ is an orthonormal basis in $L^{2}(d \mu)$ and $0 \leq p_{k}^{N} \leq 1$. A classical example of such correlation kernel is that of the GUE eigenvalue process denoted by

$$
K_{0}^{N}(x, y)=\sum_{k=0}^{N-1} \varphi_{k}(x) \varphi_{k}(y),
$$


where $\varphi_{k}(x)=\sqrt{\frac{\pi \sqrt{N}}{\sqrt{2}}} h_{k}\left(x \frac{\pi \sqrt{N}}{\sqrt{2}}\right) e^{-\left(x \frac{\pi \sqrt{N}}{\sqrt{2}}\right)^{2} / 2}$ and $h_{k}$ are the normalized Hermite polynomials with respect to the weight $e^{-x^{2}}$ on $\mathbb{R}$. The parameter $N \in \mathbb{N}$ is the dimension of the matrix and the variance of the entries is scaled so that the eigenvalue density at the origin is also of order $N$ as $N \rightarrow \infty$. For some background on the GUE process, we refer to [30], Chapters 2-5, and to Appendix A for a collection of standard facts on the asymptotics of the Hermite polynomials and the GUE kernel. In the following, our main interest is in determinantal processes whose correlation kernels are modifications of $K_{0}^{N}$, in the sense that, instead of taking the spectrum $p_{k}^{N}=\mathbb{1}_{k<N}$, we assume that $k \mapsto p_{k}^{N}$ is a function which decays smoothly. The following class of functions will be called shapes:

$$
\begin{gathered}
\mathfrak{F}=\left\{\Psi: \mathbb{R} \rightarrow[0,1] \mid \Psi^{\prime} \leq 0\right. \text { is Riemann integrable, } \\
\left.\Psi \in L^{1}(0, \infty) \text { and }(1-\Psi) \in L^{1}(-\infty, 0)\right\}
\end{gathered}
$$

and, for any $\Psi \in \mathfrak{F}$, we let

$$
\mathrm{B}_{\Psi}^{2}=\int_{\mathbb{R}} \Psi(x)(1-\Psi(x)) d x .
$$

DEFINITION 1.1. A modified GUE is a determinantal process on $\mathbb{R}$ (with respect to the Lebesgue measure) whose correlation kernel $K_{\Psi, \alpha}^{N}$ is

$$
K_{\Psi, \alpha}^{N}(x, y)=\sum_{k=0}^{\infty} \Psi\left(\frac{k-N}{\tau N^{\alpha}}\right) \varphi_{k}(x) \varphi_{k}(y),
$$

where $\Psi \in \mathfrak{F}, N>0, \alpha \in(0,1)$ and $\tau>0$. In the sequel, the parameter $\alpha$ is called the modification scale, $\tau$ is called the temperature and we assume that the shape $\Psi$ is normalized so that $\mathrm{B}_{\Psi}^{2}=1$.

Note that he kernel $K_{\Psi, \alpha}^{N}$ is not reproducing, so that the number particles in the configuration, denoted by \#, is random. Moreover, a simple computation shows that $\mathbb{E}_{K_{\Psi, \alpha}^{N}}[\#] \sim N$ and the density of particles at the origin is also of order $N$ (global scaling). According to [21], Theorem 7, another correlation kernel for the modified GUE is given by

$$
K(x, y)=\sum_{k=0}^{\infty} I_{k}^{N} \varphi_{k}(x) \varphi_{k}(y)
$$

where $I_{k}^{N}$ are independent Bernoulli random variables with $\mathbb{E}\left[I_{k}^{N}\right]=\Psi\left(\frac{k-N}{\tau N^{\alpha}}\right)$. Hence, the modified ensembles are more random than the GUE and the amount of extra randomness may be estimated by a Riemann sum approximation:

$$
\operatorname{Var}_{K_{\Psi, \alpha}^{N}}[\#]=\sum_{k=0}^{\infty} \operatorname{Var}\left[I_{k}^{N}\right]=\sum_{k=0}^{\infty} \Psi\left(\frac{k-N}{\tau N^{\alpha}}\right)\left(1-\Psi\left(\frac{k-N}{\tau N^{\alpha}}\right)\right) \sim \tau N^{\alpha} \mathrm{B}_{\Psi}^{2} .
$$


Heuristically, it means that the more the spectrum of the correlation kernel is modified, the more disorder is forced into the system. So we expect that, for large modifications, the modified ensembles behave like the Poisson process rather than like the GUE.

REMARK 1.2. According to formula (1.7), for any $\sigma>0$, the shapes $\Psi$ and $\Psi_{\sigma}(t)=\Psi(t / \sigma)$ define the same modified GUE at different temperatures. Moreover, by formula (1.6), $\mathrm{B}_{\Psi_{\sigma}}^{2}=\sigma \mathrm{B}_{\Psi}^{2}$ and the condition $\mathrm{B}_{\Psi}^{2}=1$ fixes the temperature $\tau>0$ so that

$$
\operatorname{Var}_{K_{\Psi, \alpha}^{N}}[\#]=\tau N^{\alpha}+\underset{N \rightarrow \infty}{o}(1)
$$

Our interest in determinantal processes with correlation kernels of the form (1.7) is mainly motivated by the following example that we call the MNS ensemble. In [29], motivated by the physics of mesoscopic conductors, Moshe, Neuberger and Shapiro introduced an ensemble of unitary invariant Hermitian matrices whose eigenvalue distribution interpolates between the GUE and the Poisson process. This model was rigorously analyzed in [23] and it was proved that its grand canonical version is a determinantal process with correlation kernel on $\mathbb{R}$ given by

$$
K_{\psi, \alpha}^{N}(x, y)=\sum_{k=0}^{\infty} \frac{\varphi_{k}(x) \varphi_{k}(y)}{1+e^{(k-N) / \tau N^{\alpha}}}
$$

for some $\tau>0$ and $0<\alpha<1$. So the MNS ensemble is a modified GUE with shape $\psi(t)=\left(1+e^{t}\right)^{-1}$. Moreover, this model has two natural interpretations.

First, since the rescaled Hermite functions $\left(\varphi_{k}\right)_{k \geq 0}$ are the eigenfunctions of the Schrödinger operator $-\Delta+\frac{\pi^{2} N}{2} x^{2}$ on $\mathbb{R}$, the MNS process describes a grand canonical system of free fermions at positive temperature confined in a quadratic external well. Note that the probability that the $k$ th state of this harmonic oscillator is occupied is equal to the Fermi factor $\left(1+e^{(k-N) / T}\right)^{-1}$ where $T=N^{\alpha} \tau$ is the temperature of the system. Thus, the classical GUE corresponds to the ground state of the system. Namely, taking the temperature to zero [i.e., the limit $\tau \rightarrow 0$ in (1.10)], one recovers the GUE kernel $K_{0}^{N},(1.4)$. On the other hand, for large temperature (i.e., taking $\tau \rightarrow \infty$ ), the kernel degenerates to that of a Poisson process on $\mathbb{R}$. Therefore, at a heuristic level, the MNS ensemble interpolates between Poisson and random matrix statistics. We shall prove that such a transition occurs for smooth mesoscopic linear statistics of the process and analyze the critical regime.

The kernel (1.10) also occurs in connection with the KPZ equation (KardarParisi-Zhang), where it is related to the crossover distribution for the height function; see [2, 11, 27, 34, 35]. We are not aware of any connection between the present work and the KPZ equation. Second, in [23], it was shown that the MNS process also describes a system of Brownian particles moving on a cylinder and conditioned not to collide (by rotation invariance, the distribution of the particles 
is stationary). We have seen that the parameter $N$ is the expected number of particles and one can check that the length $\beta$ of the cylinder where the particles are diffusing is related to the temperature $T$ of the free Fermions by $\beta=4 \sinh \left(T^{-1}\right)$. This particle system is expected to behave like Dyson's Brownian motion [3, 14], and this provides another heuristic description of the transition. Namely, at small scales, the particles remain roughly independent, while when $\beta$ gets large the trajectories start regularizing because of the noncolliding constraints until eventually the joint law of the particles becomes the same as that of GUE eigenvalues.

In general, one can think of $\Psi\left(\frac{k-N}{\tau N^{\alpha}}\right)$ as the probability that the $k$ th state of a quantum system is occupied. Then $1-\Psi$ corresponds to the distribution function of a probability measure on $\mathbb{R}$ and we denote by $\Phi=-\Psi^{\prime}$ the corresponding density. For technical reasons, it will be easier to consider the following subclass of shapes:

$$
\mathfrak{E}=\left\{\Psi \in \mathfrak{F} \mid \Phi(x)=-\Psi^{\prime}(x) \leq e^{-c|x|} \text { for some } c>0\right\},
$$

which contains the MNS shape $\psi(t)=\left(1+e^{t}\right)^{-1}$.

In random matrix theory, it is well known that there are different scalings to look at the eigenvalues. The global or macroscopic scale refers to the whole eigenvalue process. On the other hand, the local or microscopic scale is that of individual eigenvalues, that is, the gaps between consecutive eigenvalues are of order 1 . At this ultimate scale, in the Hermitian case, universality means that the rescaled point process converges in the bulk to the celebrated sine process. Any scale in between is called mesoscopic. In other words, a mesoscopic random variable is a function of the point process which depends on a growing fraction of the total number of particles. A typical example of such observables is the following class of linear statistics.

DEFINITION 1.3. Given a point process $\Xi$ with density of order $N$ at the origin and a function $f: \mathfrak{X} \rightarrow \mathbb{C}$ with compact support, for any $0<\delta<1$, we define

$$
f_{\delta}(x)=f\left(x N^{\delta}\right)
$$

and we call a mesoscopic linear statistic the random variable

$$
\Xi f_{\delta}=\sum_{i} f\left(X_{i} N^{\delta}\right) \text {. }
$$

In the following, the parameter $\delta$ is called the scale.

We will investigate the asymptotic distribution of mesoscopic linear statistics of the modified GUEs. Note that since the density at the origin is of order $N$, we have

$$
\mathbb{E}_{K_{\Psi, \alpha}^{N}}\left[\Xi f_{\delta}\right] \sim N^{1-\delta} \int_{\mathbb{R}} f(x) d x .
$$


If $\delta<1$, this expectation diverges as $N \rightarrow \infty$ and it is natural to consider centered linear statistics instead,

$$
\widetilde{\Xi} f_{\delta}=\sum_{i} f\left(X_{i} N^{\delta}\right)-\mathbb{E}_{K_{\Psi, \alpha}^{N}}\left[\Xi f_{\delta}\right]
$$

For any random variable $Z$ with a well-defined Laplace transform, its cumulants $C^{n}[Z]$ are given by the power series

$$
\log \mathbb{E}\left[e^{t Z}\right]=\sum_{n=1}^{\infty} \mathrm{C}^{n}[Z] \frac{t^{n}}{n !} .
$$

For determinantal processes, it turns out that there are explicit formulae, in terms of the correlation kernel $K$, for the cumulants of a linear statistic $\Xi f=\sum f\left(X_{i}\right)$. Taking $g(x)=e^{t f(x)}-1$ for some function $f \in C(\mathfrak{X})$ with compact support and $t \in \mathbb{R}$ in equation (1.2), we see that

$$
\mathbb{E}_{K}\left[e^{t \Xi f}\right]=\operatorname{det}\left[\mathrm{I}+\mathcal{K}\left(e^{t f(x)}-1\right)\right] .
$$

Since the operator $\mathcal{K}$ is assumed to be locally trace-class, the RHS of this equation is a Fredholm determinant and taking logarithm (see, for instance, [37], Chapter 3), we obtain

$$
\begin{aligned}
\log \mathbb{E}_{K}\left[e^{t \Xi f}\right] & =\operatorname{Tr}\left[\log \left(\mathrm{I}+\mathcal{K}\left(e^{t f(x)}-1\right)\right)\right] \\
& =\sum_{l=1}^{\infty} \frac{(-1)^{l+1}}{l} \operatorname{Tr}\left[\left(\mathcal{K}\left(e^{t f(x)}-1\right)\right)^{l}\right] .
\end{aligned}
$$

If we expand $e^{t f(x)}-1=\sum f(x)^{n} \frac{t^{n}}{n !}$ and use linearity of $\operatorname{Tr}$, we deduce that the cumulants of the random variable $\Xi f$ are given by

$$
\mathrm{C}_{K}^{n}[\Xi f]=\sum_{l=1}^{n} \frac{(-1)^{l+1}}{l} \sum_{\substack{m_{1}, \ldots, m_{l} \geq 1 \\ m_{1}+\cdots+m_{l}=n}} \frac{n !}{m_{1} ! \cdots m_{l} !} \operatorname{Tr}\left[f^{m_{1}} K \cdots f^{m_{l}} K\right],
$$

where we interpret $f^{m_{j}}$ as multiplication operators acting on $L^{2}(\mathfrak{X}, d \mu)$. In particular, we have

$$
\begin{aligned}
& \operatorname{Tr}\left[f^{m_{1}} K \cdots f^{m_{\ell}} K\right] \\
& \quad=\int_{\mathfrak{X}^{\ell}} f\left(x_{1}\right)^{m_{1}} K\left(x_{1}, x_{2}\right) \cdots f\left(x_{\ell}\right)^{m_{\ell}} K\left(x_{\ell}, x_{1}\right) \prod_{j<\ell} d \mu\left(x_{j}\right),
\end{aligned}
$$

so that, provided the precise asymptotics of the correlation kernel $K$ is available, we may deduce from formula (1.16) the limit law of the linear statistic $\Xi f$. For instance, we get a CLT with variance $\sigma^{2}$ if, for any $n>2$,

$$
\lim _{N \rightarrow \infty} C_{K}^{n}[\Xi f]=0 \text { and } \lim _{N \rightarrow \infty} C_{K}^{2}[\Xi f]=\sigma^{2}
$$


A composition of $n \in \mathbb{N}$ is a tuple $\mathbf{m}=\left(m_{1}, m_{2}, \ldots, m_{\ell}\right)$ of positive integers such that $|\mathbf{m}|=m_{1}+\cdots+m_{\ell}=n$, where $\ell=\ell(\mathbf{m})$ is called the length of $\mathbf{m}$. Using the notation

$$
\left(\begin{array}{c}
n \\
\mathbf{m}
\end{array}\right)=\frac{n !}{\prod_{j} \mathbf{m}_{j} !} \quad \text { and } \quad \mathbf{M}(\mathbf{m})=\frac{(-1)^{\ell+1}}{\ell}\left(\begin{array}{c}
n \\
\mathbf{m}
\end{array}\right),
$$

it will be convenient to rewrite formula (1.16) as

$$
\mathrm{C}_{K}^{n}[\Xi f]=\sum_{|\mathbf{m}|=n} \mathbf{M}(\mathbf{m}) \operatorname{Tr}\left[f^{m_{1}} K \cdots f^{m_{\ell}} K\right] .
$$

1.3. Main results. In this section, we summarize the main results of Sections 3 and 4 about the asymptotics of sufficiently smooth linear statistics of the modified GUEs. For any function $f \in L^{1} \cap L^{2}(\mathbb{R})$, we define its Fourier transform:

$$
\hat{f}(u)=\int_{\mathbb{R}} f(x) e^{-i 2 \pi x u} d x .
$$

We let $H^{1 / 2}(\mathbb{R})$, respectively, $H^{1}(\mathbb{R})$, be the (homogeneous) Sobolev spaces of real-valued $L^{2}$-functions equipped with the norms

$$
\begin{aligned}
\|f\|_{H^{1 / 2}}^{2} & =\int_{\mathbb{R}}|\hat{f}(u)|^{2}|u| d u=\frac{1}{4 \pi^{2}} \iint_{\mathbb{R}^{2}}\left|\frac{f(x)-f(y)}{x-y}\right|^{2} d x d y, \\
\|f\|_{H^{1}}^{2} & =\int_{\mathbb{R}}|\hat{f}(u)|^{2}|u|^{2} d u=\frac{1}{4 \pi^{2}} \int_{\mathbb{R}^{\prime}}\left|f^{\prime}(x)\right|^{2} d x .
\end{aligned}
$$

We also denote by $C_{0}(\mathbb{R}), H_{0}^{1 / 2}(\mathbb{R}), H_{0}^{1}(\mathbb{R})$, etc., the corresponding subspaces of compactly supported functions.

THEOREM 1.4. For any parameters $0<\delta<\alpha<1$ and for any bounded function $f \in H_{0}^{1 / 2}(\mathbb{R})$,

$$
\operatorname{Var}_{K_{\Psi, \alpha}^{N}}\left[\Xi f_{\delta}\right]=\frac{\tau}{2} N^{\alpha-\delta} \int_{\mathbb{R}} f(x)^{2} d x+\underset{N \rightarrow \infty}{o}\left(N^{\alpha-\delta}\right) .
$$

This asymptotics implies the following classical CLT as $N \rightarrow \infty$ :

$$
N^{-\frac{\alpha-\delta}{2}} \tilde{\Xi} f_{\delta} \Rightarrow \mathcal{N}\left(0, \tau\|f\|_{L^{2}}^{2} / 2\right) \text {. }
$$

PROOF. The asymptotic expansion of the variance is proved in Section 3.1. Then the CLT (1.23) follows directly from the estimates (1.13) and (1.22) by applying Theorem 1 in [40].

Hence, we will call the set $\{\delta \in[0,1): \delta<\alpha\}$ the Poisson scales because the variance of any linear statistic is diverging in this regime. Viewing the process $\widetilde{\Xi},(1.14)$, as a random distribution acting on $C_{0}^{\infty}(\mathbb{R})$, Theorem 1.4 implies 
that, once normalized, it converges at any scale $\delta<\alpha$ to a white noise with intensity $\tau / 2$, that is, a centered Gaussian field $\Xi_{\infty}$ on the real line with covariance

$$
\mathbb{E}\left[\Xi_{\infty}(f) \Xi_{\infty}(g)\right]=\frac{\tau}{2}\langle f, g\rangle_{L^{2}(\mathbb{R})}
$$

At scales $\delta \geq \alpha$, like for the GUE, the variance of smooth statistics remains bounded. Therefore, we expect a limiting process with nontrivial correlations. Actually, by comparing linear statistics of the modified GUEs with that of the sine process, we will obtain the following CLT.

THEOREM 1.5. Let $\Psi \in \mathfrak{E}$ and $f \in H_{0}^{1 / 2}(\mathbb{R})$ be a bounded function. If $0<$ $\alpha<\delta \leq 1$, then as $N \rightarrow \infty$,

$$
\widetilde{\Xi} f_{\delta} \Rightarrow \mathcal{N}\left(0,\|f\|_{H^{1 / 2}}^{2}\right)
$$

ProOF. See Section 3.2.

Hence, we will call the set $\{\delta \in(0,1): \alpha<\delta\}$ the GUE scales by analogy with Theorem 3.6 below. The interpretation of Theorem 1.5 is that the centered modified GUEs converge weakly at any scale $\delta>\alpha$ to a Gaussian process $\Xi_{0}$ on the real line with covariance

$$
\mathbb{E}\left[\Xi_{0}(f) \Xi_{0}(g)\right]=\langle f, g\rangle_{H^{1 / 2}}
$$

In contrast to a white noise, the Gaussian process $\Xi_{0}$ is spatially correlated and self-similar as can be seen from formula (1.20). Theorems 1.4 and 1.5 imply that the modified GUEs undergo a transition from Poisson to random matrix statistics when the mesoscopic scale $\delta$ is equal to the modification scale $\alpha$ of its correlation kernel. Our next question is what happens at the critical scale? The first step is to investigate the variance of linear statistics.

THEOREM 1.6. Let $\Psi \in \mathfrak{E}$ and $f \in H_{0}^{1 / 2}(\mathbb{R})$ be a bounded function. For any scale $0<\alpha<1$,

$$
\lim _{N \rightarrow \infty} \operatorname{Var}_{K_{\Psi, \alpha}^{N}}\left[\Xi f_{\alpha}\right]
$$

$$
\begin{aligned}
& =2 \tau^{\prime} \int_{\mathbb{R}}|\hat{f}(u)|^{2} \int_{\mathbb{R}} \Psi(t)\left(1-\Psi\left(t+u / \tau^{\prime}\right)\right) d u d t \\
& =2 \tau^{\prime} \int f(x)^{2} d x+\frac{1}{4 \pi^{2}} \iint\left|\frac{f(x)-f(y)}{x-y}\right|^{2}\left|\hat{\Phi}\left(\tau^{\prime}(x-y)\right)\right|^{2} d x d y,
\end{aligned}
$$

where $\Phi=-\Psi^{\prime}$ and the parameter $\tau^{\prime}=\frac{\tau}{4}>0$. 
ProOF. See Appendix B.

Since $\Phi$ is a probability distribution function, it is clear that formula (1.24) interpolates between $\|f\|_{H^{1 / 2}}^{2}$ as $\tau \rightarrow 0$, respectively, $2 \tau^{\prime}\|f\|_{L^{2}}^{2}$ as $\tau \rightarrow \infty$. Hence, we recover the variances of Theorem 1.4 and Theorem 1.5, respectively. In analogy, we would also expect Gaussian fluctuations when $\delta=\alpha$. Surprisingly, at this critical scale, the cumulants of linear statistics of the modified ensembles have nontrivial limits. In order to formulate our main result, we need to introduce additional notation. Let

$$
\mathbb{R}_{<}^{n}=\left\{x \in \mathbb{R}^{n}: x_{1}<\cdots<x_{n}\right\}
$$

and for any $t \in \mathbb{R}$,

$$
\mathbb{R}_{t}^{n}=\left\{x \in \mathbb{R}^{n}: x_{1}+\cdots+x_{n}=t\right\} .
$$

For any composition $\mathbf{m}$ and for any $k \leq \ell(\mathbf{m})$, we let

$$
\bar{m}_{k}=m_{1}+m_{2}+\cdots+m_{k} .
$$

For any $u \in \mathbb{R}^{|\mathbf{m}|}$, we define

$$
\Lambda_{i, s}^{\mathbf{m}}(u)=\sum_{j=1}^{\bar{m}_{i}} u_{j}-\sum_{j=1}^{\bar{m}_{s}} u_{j}= \begin{cases}u_{\bar{m}_{s}+1}+\cdots+u_{\bar{m}_{i}} & \text { if } s<i, \\ -u_{\bar{m}_{i}+1}-\cdots-u_{\bar{m}_{s}} & \text { if } i<s, \\ 0 & \text { if } i=s,\end{cases}
$$

so that $\Lambda^{\mathbf{m}}=\left(\Lambda_{i, s}^{\mathbf{m}}\right)$ is a $\ell(\mathbf{m}) \times \ell(\mathbf{m})$ antisymmetric matrix. If $\sigma \in \mathbb{S}(n)$ is a permutation of $[n]=\{1, \ldots, n\}$, we will use the shorthand notation $\sigma u=$ $\left(u_{\sigma(1)}, \ldots, u_{\sigma(n)}\right)$ and we define $\mathfrak{s}(\sigma)=\arg \min (\sigma) \in[n] \times[n-1] \times \cdots \times[1]$ as follows. That is, for any $l \in[n]$, the number $\mathfrak{s}_{l}(\sigma)$ is given implicitly by the relation

$$
\sigma\left(\mathfrak{s}_{l}\right)=\min \{\sigma(j): j=1, \ldots, l\} .
$$

For any $\tau>0$ and any composition $\mathbf{m}$ of $n \geq 2$, let

$$
\mathrm{G}_{\tau}^{\mathbf{m}}(u, x)=\sum_{\sigma \in \mathbb{S}(n)} \max _{i \leq \ell}\left\{\Lambda_{i, \mathfrak{s}_{\ell}}^{\mathbf{m}}(u)-\tau\left(x_{\sigma(i)}-x_{\sigma(\mathfrak{s} \ell)}\right)\right\},
$$

where we used the shorthand notation $\ell=\ell(\mathbf{m})$ and $\mathfrak{s}_{\ell}=\mathfrak{s}_{\ell(\mathbf{m})}(\sigma)$. Note that, since $\Lambda_{\mathfrak{s}_{\ell}, \mathfrak{s}_{\ell}}^{\mathbf{m}}(u)=0$, the functions $G_{\tau}^{\mathbf{m}}(u, x)$ are nonnegative on $\mathbb{R}^{n} \times \mathbb{R}_{<}^{n}$. Finally, for any function $\Psi \in \mathfrak{F}$, let $\mathrm{B}_{\Psi}^{1}=0$ and, for all $n \geq 2$,

$$
\mathrm{B}_{\Psi}^{n}=\sum_{k=0}^{n-1} b_{k}^{n} \int_{\mathbb{R}} x \Phi(x) \Psi(x)^{k}(1-\Psi(x))^{n-1-k} d x
$$


where the coefficients $b_{k}^{n}$ are given by

$$
b_{k}^{n}=\sum_{l=1}^{k+1}(-1)^{l+1}\left(\begin{array}{c}
n-l \\
k+1-l
\end{array}\right) \sum_{\substack{|\mathbf{m}|=n \\
\ell(\mathbf{m})=l}}\left(\begin{array}{l}
n \\
\mathbf{m}
\end{array}\right) .
$$

Note that since $b_{0}^{2}=-b_{1}^{2}=1$, formula (1.29) implies that

$$
\mathrm{B}_{\Psi}^{2}=\int_{\mathbb{R}} x \Phi(x)(1-2 \Psi(x)) d x .
$$

Then, using that for any shape $\Psi \in \mathfrak{F}$, we have

$$
\Phi(x)(1-2 \Psi(x))=-\frac{d}{d x}\{\Psi(x)(1-\Psi(x))\},
$$

we recover formula (1.6) by an integration by part.

THEOREM 1.7. Let $0<\alpha<1, \Psi \in \mathfrak{F}^{*}$, and consider the determinantal process with kernel $K_{\Psi, \alpha}^{N}$. For any bounded function $f \in H_{0}^{1 / 2}(\mathbb{R})$, the centered linear statistic $\widetilde{\Xi} f_{\alpha}$ converges in distribution as the density $N \rightarrow \infty$ to a random variable denoted $\Xi_{\Psi, \tau / 4} f$. Moreover, if $f \in H_{0}^{1}(\mathbb{R})$, then the cumulants of $\Xi_{\Psi, \tau} f$ are given by for all $n \geq 2$,

$$
\begin{aligned}
\mathrm{C}^{n}\left[\boldsymbol{\Xi}_{\Psi, \tau} f\right]= & 2 \tau \mathrm{B}_{\Psi}^{n} \int_{\mathbb{R}} f(t)^{n} d t-2 \sum_{|\mathbf{m}|=n} \mathrm{M}(\mathbf{m}) \\
& \times \int_{\mathbb{R}_{0}^{n}} d u \int_{\mathbb{R}_{<}^{n}} d x \Re\left\{\prod_{i=1}^{n} \hat{f}\left(u_{i}\right) \Phi\left(x_{i}\right)\right\} \mathrm{G}_{\tau}^{\mathbf{m}}(u, x) .
\end{aligned}
$$

Proof. See Section 4.2.

It seems to be a difficult problem to recover the Laplace transform of the random variable $\Xi_{\Psi, \tau} f$ from formula (1.31). In fact, we are only able to infer a few properties of its distribution, such as the fact that it is not Gaussian and the dependence in the temperature $\tau$.

THEOREM 1.8. For any shape $\Psi \in \mathfrak{F}$ and any $\tau>0$, the random process $\Xi_{\Psi, \tau}$ which arise from the limit of a modified ensemble at the critical scale $\delta=\alpha$ is not Gaussian.

Surprisingly, the proof of Theorem 1.8 is rather involved and relies on two separate arguments. First, we show that, if there exists $n>2$ such that the parameter $\mathrm{B}_{\Psi}^{n} \neq 0$, then the random process $\Xi_{\Psi, \tau}$ is not Gaussian and that, unexpectedly, this criterion is satisfied by all modified GUEs except the MNS ensemble. 
PROPOSITION 1.9. The only function $\Psi \in \mathfrak{F}$ which satisfies the conditions $\mathrm{B}_{\Psi}^{n}=0$ for all $n>2$ is the MNS shape $\psi(t)=1 /\left(1+e^{t}\right)$.

\section{ProOF. See Section 4.3.}

This special property of the MNS ensemble might be related to the fact that this process originates from free fermions and it would be interesting to know whether it has any physical interpretation. From a probabilistic perspective, the main consequence of Proposition 1.9 is that we need another argument to show that the process $\Xi_{\psi, \tau}$ is not Gaussian. To this end, we show in Section 4.4 that the Schwartz function $y(x)=2 e^{-\epsilon \pi x^{2}} \cos (2 \pi x)$ satisfies if $\epsilon$ is sufficiently small:

$$
\mathrm{C}^{4}\left[\Xi_{\psi, \tau} y\right] \neq 0 \text {. }
$$

We must look at the 4 th cumulant since $C^{3}\left[\Xi_{\psi, \tau} f\right]=0$ for any test function because of certain symmetries; see Proposition 4.19. Let us also point out that there is nothing special about the function $y(x)$ except that it is simple enough to provide a good example.

To conclude, we have seen that the MNS ensemble describes noncolliding Brownian motions in a cylindrical geometry. In this case, the diagram of Figure 1 shows that the particle statistics at a given mesoscopic scale $\delta$ exhibit a sharp transition from Poisson to GUE at time $N^{-\delta}$. This situation is similar to that of the Dyson's Brownian motion which was investigated in [14]. However, the transitions are different: for the MNS ensemble, there is no intermediate regime which depends on the test function $f$ and unexpectedly the critical fluctuations are not

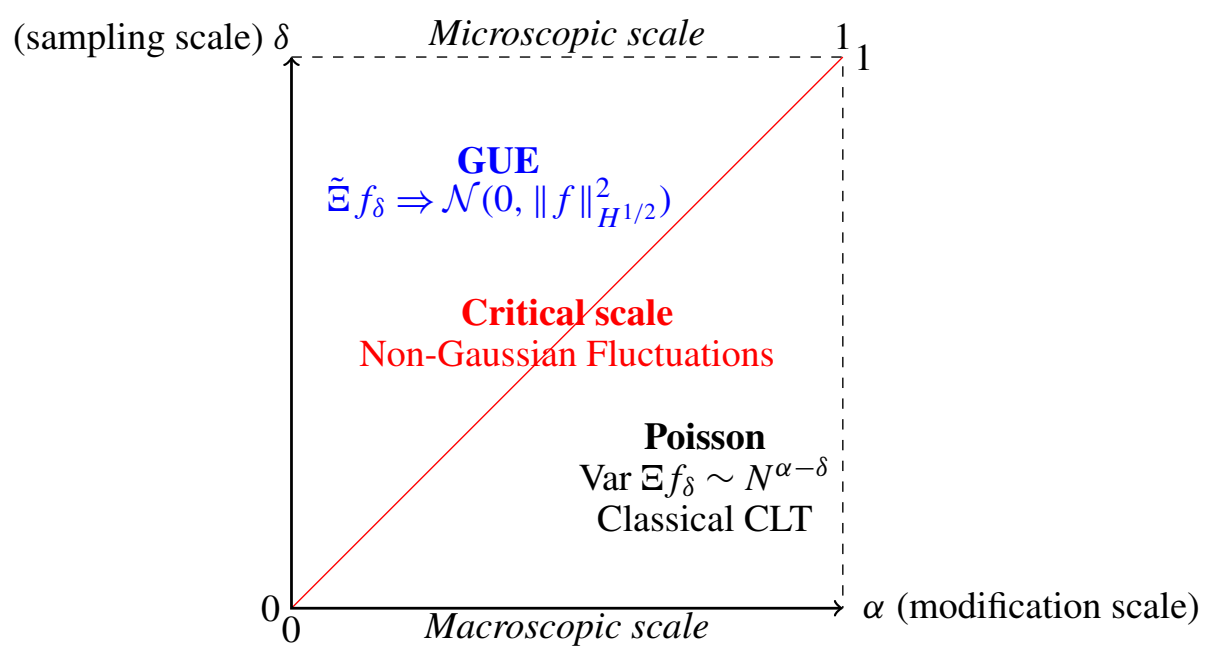

FIG. 1. Diagram of the fluctuations for a smooth linear statistics of a modified GUE as a function of the scale $\delta$ and the modification scale $\alpha$. 
Gaussian. In both interpretations, either as noncolliding Brownian motions, or as a grand canonical ensemble of free fermions, it is not clear why this phenomenon occurs at a certain relation between the sampling scale of the linear statistic and the size of cylinder, or the temperature. Actually, it would be very interesting to get another description than Theorem 1.7 of the random field $\Xi_{\psi, \tau}$ which arises at the critical scale in order to understand how the spatial correlations disappear in the transition from the $H^{1 / 2}$-Gaussian noise $\Xi_{0}$ to the white noise $\Xi_{\infty}$. Another point of interest would be to understand how the processes $\Xi_{\Psi, \tau}$ depend on the shape $\Psi$ and why the MNS Ensemble appears to be special.

1.4. Outline of the main ideas. For determinantal processes, a general strategy to obtain a CLT for linear statistics is to use formula (1.16) to show that all cumulants of order $\geq 3$ converge to zero. This approach has been applied to many classical models in 1 or 2 dimensions using a wide range of techniques; see, for example, [1, 8-10, 32, 33, 39, 40]. In this paper, it is applied to the modified ensembles of Definition 1.1. To the authors' knowledge, the results of this paper at the critical scale provide the first example of a determinantal point process for which the fluctuations of a mesoscopic linear statistic are not Gaussian in the limit. At the global scale, non-Gaussian limits can be obtained for unitary invariant Hermitian matrix models with several cuts, but the mechanism is different. In fact, it was recently proved in [25] that, at mesoscopic scales, such models are also described in the large $N$ limit by the $H^{1 / 2}$-Gaussian noise $\Xi_{0}$ appearing in Theorem 1.5. To prove Theorems 1.5 and 1.7, we use a perturbative approach which consists in comparing the correlation kernels of two processes to establish that a given linear statistic $\Xi f$ has the same limit for both ensembles as their density $N \rightarrow \infty$. To this end, we will use the following definition.

Definition 1.10. Two families of kernels $\left(K^{N}\right)_{N>0}$ and $\left(L^{N}\right)_{N>0}$ defined on the same space $\mathfrak{X}$ are asymptotically equivalent (written as $K^{N} \cong L^{N}$ ) if, for any $\ell \in \mathbb{N}$ and any functions $f_{1}, \ldots, f_{\ell} \in C_{0}^{\infty}(\mathfrak{X})$,

$$
\lim _{N \rightarrow \infty} \operatorname{Tr}\left[f_{1} K^{N} \cdots f_{\ell} K^{N}\right]=\lim _{N \rightarrow \infty} \operatorname{Tr}\left[f_{1} L^{N} \cdots f_{\ell} L^{N}\right] .
$$

Definition 1.10 does not apply only to correlation kernels, but to all families of kernels which are locally trace-class. However, if both $K^{N}$ and $L^{N}$ define determinantal processes and $K^{N} \cong L^{N}$, by formula (1.19), these processes have the same limit. For instance, Theorem 1.5 is proved by showing that the kernel $K_{\Psi, \alpha}^{N}$ and the GUE kernel $K_{0}^{N}$ are asymptotically equivalent at any scale $\delta>\alpha$ and using the CLT for the mesoscopic GUE (see Theorem 3.6 proved in $[5,19$, 25]). On the other hand, at the critical scale, $\delta=\alpha$, the kernels $K_{\Psi, \alpha}^{N}$ are not asymptotically equivalent to any kernel which has been studied previously and we will need to compute the limits of the cumulants explicitly. If $\Psi \in \mathfrak{F}$, recall that $\Phi=-\Psi^{\prime}$ and define $\xi_{k} \in(0,1)$ such that by the mean-value theorem: 
$\frac{1}{\tau N^{\alpha}} \Phi\left(\frac{k+\xi_{k}}{\tau N^{\alpha}}\right)=\Psi\left(\frac{k}{\tau N^{\alpha}}\right)-\Psi\left(\frac{k+1}{\tau N^{\alpha}}\right)$ for any $k \in \mathbb{Z}$. Then, if $N, \tau, \Gamma>0, \alpha \in(0,1)$, and $\eta$ is a nondecreasing function, we define the kernel

$$
L_{\Psi, \eta}^{N}(x, y)=\frac{1}{\tau N^{\alpha}} \sum_{|k| \leq \Gamma N^{\alpha}} \Phi\left(\frac{k+\xi_{k}}{\tau N^{\alpha}}\right) \frac{\sin [2 \pi \eta(k)(x-y)]}{\pi(x-y)} .
$$

Lemma 4.1 below shows that the kernel $L_{\Psi, \eta}^{N}$ defines a determinantal process on $\mathbb{R}$ and the next proposition implies that, at sufficiently small scales, mesoscopic linear statistics of the critical modified GUE with shape $\Psi$ and the determinantal process with kernel $L_{\Psi, \eta}^{N}$ have the same limit.

Proposition 1.11. Let $\Psi \in \mathfrak{E}, \eta(k)=N^{1-\alpha} \sqrt{1+k / N} / 2$ and $\Gamma=$ $(\log N)^{2}$. For all $1 / 3<\alpha<1$, the rescaled correlation kernel of the modified GUE satisfies $N^{-\alpha} K_{\Psi, \alpha}^{N}\left(x N^{-\alpha}, y N^{-\alpha}\right) \cong L_{\Psi, \eta}^{N}(x, y)$ in the sense of Definition 1.10.

PROOF. See Section 4.1.

Proposition 1.11, combined with the analysis of the determinantal process with correlation kernel $L_{\Psi, \eta}^{N}$ performed in Section 4.1, implies Theorem 1.7 in the regime $1 / 3<\alpha<1$. The main technical challenge of this argument is to obtain the asymptotic expansion of the GUE kernel at all mesoscopic scales. We show in Appendix A that, if $M \sim N$, then for any $\delta>0$,

$$
N^{-\delta} K_{0}^{M}\left(x N^{-\delta}, y N^{-\delta}\right)=\frac{\sin \left[\pi N^{1 / 2-\delta} \sqrt{M}(x-y)\right]}{\pi(x-y)}+\underset{N \rightarrow \infty}{O}\left(N^{1-3 \delta}\right)
$$

uniformly for all $x, y$ in compact subsets of $\mathbb{R}$. Note that when $\delta \leq 1 / 3$, the error term in formula (A.11) does not converge to 0 , hence the restriction in Proposition 1.11. This restriction comes from the fact the GUE kernel is not asymptotically translation-invariant at large scales because of the curvature of the semicircle density and the proof of Theorem 1.7 in the general case relies on a different argument which basically consists in unfolding the point process to reduce again to the case of the kernel $L_{\Psi, \eta}^{N}$; see Proposition 4.11. The advantage of this approach is that the kernel $L_{\Psi, \eta}^{N}$ is translation-invariant and we can compute the cumulants of its linear statistics by using the method devised in [39] to prove a CLT for mesoscopic linear statistics of the circular unitary ensemble (see also Theorem 4 in [40] for an application to the sine process, as well as some generalizations). In fact, taking the parameter $\tau \rightarrow 0$ in equation (1.31), we recover Soshnikov's formula for the cumulants of mesoscopic statistics of the sine process

$$
\mathrm{C}^{n}\left[\Xi_{\Psi, 0} f\right]=2 \int_{\mathbb{R}_{0}^{n}} d u \Re\left\{\prod_{i} \hat{f}\left(u_{i}\right)\right\} \sum_{|\mathbf{m}|=n} \mathbf{M}(\mathbf{m}) \mathrm{G}_{0}^{\mathbf{m}}(u),
$$

where $\mathrm{G}_{0}^{\mathbf{m}}(u)=\sum_{\pi \in \mathbb{S}(n)} \max \left\{u_{\pi(1)}+\cdots+u_{\pi\left(\bar{m}_{1}\right)}, \ldots, u_{\pi(1)}+\cdots+u_{\pi\left(\bar{m}_{\ell-1}\right)}, 0\right\}$. Then the main combinatorial lemma of [39] implies that for any $n>2$, 
$\sum M(\mathbf{m}) G_{0}^{\mathbf{m}}(u)=0$ so that the process $\Xi_{\Psi, 0}$ (which is independent of $\Psi$ ) is Gaussian. The details of the computations are given in the proof of Proposition 4.15. For the modified ensembles, it is worth noting that there is no counterpart of the main combinatorial lemma, that is, for generic points $u \in \mathbb{R}_{0}^{n}$ and $x \in \mathbb{R}_{<}^{n}$, $\sum \mathrm{M}(\mathbf{m}) \mathrm{G}_{\tau}^{\mathbf{m}}(u, x) \neq 0$ for any $\tau>0$ and $n \neq 3$. With Lemma C.1, we use this fact to prove equation (1.32). The bottom line is that the combinatorial structure behind the cumulants of the sine process, which corresponds to the continuous counterpart of the strong Szegô theorem, is very sensitive. In general, CLTs with bounded variance are due to special correlations which are rather sensitive to perturbations such as small modifications of the kernel of a determinantal process. In the remainder of this section, we provide a basic example which elaborates on this fact and illustrates how asymptotic normality breaks down. Before proceeding, we will define the circular counterparts of the modified GUEs. These processes are of interest because there is no asymptotic analysis required in order to apply Soshnikov's method while they retain the same features as the modified GUEs. In the sequel, we let $\mathbb{T}=\left[-\frac{1}{2}, \frac{1}{2}\right]$ with the boundary points identified.

Definition 1.12. A modified $C U E$ is a determinantal process on $\mathbb{T}$ whose correlation kernel $K_{p}^{N}$ (with respect to the uniform measure) is of the form

$$
K_{p}^{N}(x, y)=\sum_{k \in \mathbb{Z}} p_{k}^{N} e^{i 2 \pi k(y-x)}
$$

where $p_{-k}^{N}=p_{k}^{N}$ so that the integral operator $\mathcal{K}_{p}^{N}$ is self-adjoint on $L^{2}(\mathbb{T})$.

REMARK 1.13. In this section, the spectrum of the kernel $K_{p}^{N}$ is arbitrary except for the constraints $p_{k}^{N} \in[0,1]$. However, when we refer to a modified CUE in Sections 2.2, 2.3 and 4, like in Definition 1.1, it is understood that for all $k \in \mathbb{Z}$,

$$
p_{k}^{N}=\Psi\left(\frac{|k|-N}{\tau N^{\alpha}}\right)
$$

for a shape $\Psi \in \mathfrak{F}$ such that $\mathrm{B}_{\Psi}^{2}=1$, a modification scale $\alpha \in(0,1)$, and a temperature $\tau>0$.

A special case is the so-called Dyson's Circular Unitary Ensemble (CUE) which has the correlation kernel

$$
K^{N}(x, y)=\sum_{|k| \leq N} e^{i 2 \pi k(x-y)}=\frac{\sin ((2 N+1) \pi(x-y))}{\sin (\pi(x-y))} .
$$

This process describes the eigenvalues of a random matrix distributed according to the Haar measure on the group $\mathcal{U}(2 N+1)$. The cumulants of its linear statistics were computed explicitly in [39] and a similar computation yields the following formula in the case of the modified CUEs. 
LEMMA 1.14. For any continuous function $f: \mathbb{T} \rightarrow \mathbb{R}$,

$$
\mathrm{C}_{K_{p}^{N}}^{n}[\Xi f]=\sum_{u \in \mathbb{Z}_{0}^{n}} \prod_{j} \hat{f}\left(u_{j}\right) \sum_{|\mathbf{m}|=n} \mathbf{M}(\mathbf{m}) \sum_{k \in \mathbb{Z}} \prod_{j=0}^{\ell(m)-1} p_{k+u_{1}+\cdots+u_{\bar{m}_{j}}}^{N},
$$

where $\hat{f}(k)$ is the kth Fourier coefficient of $f$.

PROOF. See Section 2.1.

In particular, we can use this formula to investigate the behavior of a (global) linear statistics under some very simple modification of the spectrum of the CUE kernel. For instance, we can remove a single mode by choosing for some $0<m \leq N$,

$$
p_{k}^{N}=\mathbb{1}_{|k| \leq N}-\mathbb{1}_{|k|=N-m} .
$$

Then $K_{p}^{N}$ is still a projection kernel and, by Lemma 1.14 , the 3rd cumulant of a linear statistic is given by

$$
\begin{aligned}
\mathrm{C}_{K_{p}^{N}}^{3}[\Xi f]= & \sum_{u \in \mathbb{Z}_{0}^{3}} \prod_{i} \hat{f}\left(u_{i}\right)\left\{\sum_{k \in \mathbb{Z}} p_{k}-\frac{3}{2} \sum_{k \in \mathbb{Z}} p_{k}\left(p_{k+u_{1}}+p_{k+u_{1}+u_{2}}\right)\right. \\
& +2 \sum_{k \in \mathbb{Z}} p_{k} p_{k+u_{1}} p_{\left.k+u_{1}+u_{2}\right\} .}
\end{aligned}
$$

We can symmetrize this expression using permutations of the $u_{i}$ 's and the condition $u_{1}+u_{2}=-u_{3}$; this yields

$$
\mathrm{C}_{K_{p}^{N}}^{3}[\Xi f]=\sum_{u \in \mathbb{Z}_{0}^{3}} \prod_{i} \hat{f}\left(u_{i}\right) \sum_{k \in \mathbb{Z}} p_{k}\left\{1-3 p_{k+u_{1}}+2 p_{k+u_{1}} p_{k-u_{2}}\right\} .
$$

If we consider function $g_{j}(t)=2 \cos (2 \pi j t)+a \cos (4 \pi j t)$ with parameters $a \in \mathbb{R}$ and $j \in \mathbb{Z}_{+}$, we have

$$
\hat{g_{j}}(u)=\delta_{j}(|u|)+\frac{a}{2} \delta_{2 j}(|u|),
$$

so that the only frequencies $u \in \mathbb{Z}_{0}^{3}$ which contribute to (1.39) are given by all possible permutations of $( \pm j, \pm j, \mp 2 j)$ and an elementary computation gives

$$
\mathrm{C}_{K_{p}^{N}}^{3}\left[\Xi g_{j}\right]=3 a \sum_{k \in \mathbb{Z}} p_{k}\left\{1-2 p_{k+j}-p_{k+2 j}+2 p_{k+j} p_{k-j}\right\} .
$$

In the CUE case, when $p_{k}^{N}=\mathbb{1}_{|k| \leq N}$, it is easy to check that $\mathrm{C}_{K^{N}}^{3}\left[\Xi g_{j}\right]=0$ for all $j \in \mathbb{Z}_{+}$as we expected. However, it seems clear that for some generic choice of coefficients $0 \leq p_{k}^{N} \leq 1$, the expression (1.40) will be nonzero. For instance in the case (1.38), for any $j, m \ll N$,

$$
\mathrm{C}_{K_{p}^{N}}^{3}\left[\Xi g_{j}\right]=12 a\left(1-\mathbb{1}_{j \leq\lfloor m / 2\rfloor}\right) .
$$


Thus, if we remove a mode near the edge of the spectrum of the CUE kernel $(m<2 j)$, the linear statistics $\Xi g_{j}$ cannot be asymptotically Gaussian while its variance remains bounded:

$$
\mathrm{C}_{K_{p}^{N}}^{2}\left[\Xi g_{j}\right]=\sum_{u \in \mathbb{Z}}\left|\hat{g}_{j}(u)\right|^{2} \sum_{k \in \mathbb{Z}} p_{k}\left(1-p_{k+u}\right) \leq j\left(2+a^{2}\right) .
$$

In particular, even if the correlation kernel $K_{p}^{N}$ is reproducing, we can get nonGaussian behavior. This example also shows that it is the edge of the spectrum of the correlation kernel which is influencing the distribution of the point process, a fact which is also emphasized by [40], Theorem 4. Moreover, using Lemma 1.14, we can also check that when removing $M$ different modes at the edge of the spectrum, all cumulants are of order $M$ for large $N$. Hence, if we remove sufficiently many modes, the system begins to behave like a Poisson process when $N \rightarrow \infty$. Finally, note that according to (1.8), removing modes is comparable to smoothing the spectrum of the correlation kernel. Hence, this example illustrates why the modified ensembles of Definition 1.1 are not Gaussian at the critical scale $(\delta=\alpha)$. Actually, the strategy to obtain (1.32) is similar to this example but the computations are much more involved.

1.5. Overview of the rest of the paper. In Section 2, we begin by analyzing the modified CUEs of Definition 1.12. This setting is simpler than that of Section 1.2 and we can focus on the combinatorial structure of the cumulants. In particular, in Sections 2.2 and 2.3, we show that, if the spectrum of the kernel is given by $p_{k}^{N}=\Psi\left(\frac{|k|-N}{\tau N^{\alpha}}\right)$, then the results presented on Figure 1 hold for the modified CUEs as well. The main results of Section 1.3 are proved in Sections 3 and 4. The asymptotics of the variance in the Poisson regime, formula (1.22), is computed in Section 3.1, while in the GUE regime, Theorem 1.5 is proved in Section 3.2. Both the critical modified CUEs and GUEs are analyzed, in a common framework, in Section 4. In particular, the proof of Theorem 1.7 is divided in two steps. First, the limits of the cumulants are established in Theorem 4.4 (see also Proposition 4.13). Then the weak convergence of linear statistics is established in Corollary 4.10. In Section 4.3, we show that the random processes $\Xi_{\Psi, \tau}$ defined by Theorem 1.7 are not Gaussian and we prove the special property of the MNS ensemble (see Theorem 1.9) by computing the generating function of the coefficient $\mathrm{B}_{\Psi}^{n}$. We also prove that, as it is expected from Figure 1, the random field $\Xi_{\Psi, \tau}$ converges to a Gaussian process in both limits $\tau \rightarrow 0$ and $\tau \rightarrow \infty$; see Proposition 4.15. Finally, in Section 4.4, we show that the critical MNS ensemble is not Gaussian by verifying (1.32). All the asymptotics that are required to analyze the modified GUEs are gathered in Appendix A. In Appendix B, we prove Theorem 1.6 and, as an example, we compute the critical variance of linear statistics of the MNS ensemble. In Appendix C, we prove some technical lemmas which shows that there is no counterpart of the main combinatorial lemma for the modified ensemble. Finally, we will use the following conventions: 
- $C>0$ is a universal constant which changes from line to line.

- $x_{N} \ll z_{N}$ if $\lim _{N \rightarrow \infty} x_{N} / z_{N}=+\infty$.

- $x_{N} \sim z_{N}$ if $\lim _{N \rightarrow \infty} x_{N} / z_{N}=1$.

- $x_{N} \simeq z_{N}$ if $\lim _{N \rightarrow \infty}\left(x_{N}-z_{N}\right)=0$.

- $x_{N}=\bar{O}\left(z_{N}\right)$ if there exist $\kappa>0$ and $C>0$ such that $\left|x_{N}\right| \leq C z_{N}|\log N|^{\kappa}$.

2. Modified circular unitary ensembles. We present the counterparts of the results of Section 1.3 for the modified CUEs (see Definition 1.12). Along the way, we set up definitions and lemmas that will also be used in Sections 3 and 4. We consider circular ensembles as simplified models which are helpful to understand the combinatorial structure behind the cumulants of linear statistics of the MNS model because no asymptotic estimates are required to pass to the limit. In Section 2.1, we review the method introduced in [39]. In Section 2.2, we show that the modified CUEs exhibit the same transition as in Figure 1. Finally, in Section 2.3, we provide asymptotically equivalent kernels for the modified CUEs in the critical regime $\delta=\alpha$ and deduce a limit theorem from the results of Section 4.

2.1. Soshnikov's method: Proof of Lemma 1.14. In [40], Lemma 1, Soshnikov proved that the cumulants of linear statistics of a determinantal process are given by

$$
\mathrm{C}_{K}^{n}[\boldsymbol{\Xi} f]=\sum_{|\mathbf{m}|=n} \mathbf{M}(\mathbf{m}) \operatorname{Tr}\left[f^{m_{1}} K f^{m_{2}} \cdots f^{m_{\ell}} K\right]
$$

where $K$ is the correlation kernel of the process and the sum is over all compositions $\mathbf{m}$ of $n$ [see formula (1.19) and the above notation]. Applying this formula to a modified CUE and using elementary Fourier analysis, we obtain Lemma 1.14.

PROOF OF LEMMA 1.14. The correlation kernel of the modified CUE is $K_{p}^{N}(x, y)=\sum_{k \in \mathbb{Z}} p_{k}^{N} e^{i 2 \pi k(y-x)}$, and, by (1.17), we have for any composition $\mathbf{m}$ of $n$,

$$
\operatorname{Tr}\left[f^{m_{1}} K_{p}^{N} \cdots f^{m_{j}} K_{p}^{N}\right]=\sum_{\kappa \in \mathbb{Z}^{\ell}(\mathbf{m})} \prod_{i=1}^{\ell(\mathbf{m})} p_{\kappa_{i}}^{N} \widehat{f^{m_{i}}}\left(\kappa_{i}-\kappa_{i-1}\right),
$$

where by convention $\kappa_{0}=\kappa_{\ell(\mathbf{m})}$. Moreover, for any indices $s, r \in \mathbb{Z}$ and $m \in \mathbb{N}$,

$$
\widehat{f^{m}}(s-r)=\sum_{k \in \mathbb{Z}^{m-1}} \hat{f}\left(k_{1}-r\right) \hat{f}\left(k_{2}-k_{1}\right) \cdots \hat{f}\left(s-k_{m-1}\right) .
$$

So, if $\bar{m}_{j}=m_{1}+\cdots+m_{j}$ as in definition (1.25), we can write for any $i=$ $0, \ldots, \ell-1$,

$$
\begin{aligned}
& \widehat{f^{m_{i+1}}}\left(k_{\bar{m}_{i+1}}-k_{\bar{m}_{i}}\right) \\
& \quad=\sum \hat{f}\left(k_{\bar{m}_{i}+1}-k_{\bar{m}_{i}}\right) \hat{f}\left(k_{\bar{m}_{i}+2}-k_{\bar{m}_{i}+1}\right) \cdots \hat{f}\left(k_{\bar{m}_{i+1}}-k_{\bar{m}_{i+1}-1}\right)
\end{aligned}
$$


and if we make the change of variables $\kappa_{i}=k_{\bar{m}_{i}}$ in equation (2.1), putting everything together we obtain

$$
\operatorname{Tr}\left[f^{m_{1}} K_{p}^{N} f^{m_{2}} \cdots f^{m_{j}} K_{p}^{N}\right]=\sum_{k \in \mathbb{Z}^{n+1}: k_{0}=k_{n}} \prod_{i=1}^{n} \hat{f}\left(k_{i}-k_{i-1}\right) \prod_{i=1}^{\ell(\mathbf{m})} p_{k_{\bar{m}_{i}}}^{N} .
$$

We can also make the change of variables $u_{i}=k_{i}-k_{i-1}$ for all $i=1, \ldots, n$ in the previous sum. This maps $\left\{k \in \mathbb{Z}^{n}: k_{0}=k_{n}\right\}$ into $\left\{\left(k_{0}, u\right) \in \mathbb{Z} \times \mathbb{Z}_{0}^{n}\right\}$ and we conclude that

$$
\operatorname{Tr}\left[f^{m_{1}} K_{p}^{N} f^{m_{2}} \cdots f^{m_{j}} K_{p}^{N}\right]=\sum_{u \in \mathbb{Z}_{0}^{n}} \prod_{i} \hat{f}\left(u_{i}\right) \sum_{k_{0} \in \mathbb{Z}} \prod_{i=1}^{\ell(\mathbf{m})} p_{k_{0}+u_{1}+\cdots+u_{\bar{m}_{i}}}^{N} .
$$

Hence, Lemma 1.14 follows directly from formula (1.19).

In Section 1.4, we used Lemma 1.14 to show that a modified CUE has nonGaussian fluctuations at the macroscopic scale. In the sequel, we will use it to investigate fluctuations at mesoscopic scales. Let $f \in C_{0}(\mathbb{R}), 0<\delta<1$, and recall that $\mathbb{T}=\left[-\frac{1}{2}, \frac{1}{2}\right]$ with the endpoints identified. When the parameter $N$ is sufficiently large, the function $f\left(\cdot N^{\delta}\right)$ is supported in $\left[-\frac{1}{2}, \frac{1}{2}\right]$ and it can be extended to some function $f_{\delta} \in C(\mathbb{T})$. Then the Fourier coefficients of $f_{\delta}$ are given by, for any $u \in \mathbb{Z}$,

$$
\widehat{f_{\delta}}(u)=N^{-\delta} \hat{f}\left(u N^{-\delta}\right)
$$

so that

$$
C_{K_{p}^{N}}^{n}\left[\Xi f_{\delta}\right]=N^{-n \delta} \sum_{u \in \mathbb{Z}_{0}^{n}} \prod_{i} \hat{f}\left(u_{i} N^{-\delta}\right) \sum_{|\mathbf{m}|=n} \mathbf{M}(\mathbf{m}) \sum_{k \in \mathbb{Z}} \prod_{i=1}^{\ell(\mathbf{m})} p_{k+u_{1}+\cdots+u_{\bar{m}_{i}}}^{N} .
$$

2.2. Central limit theorems. For now on, we will assume that the spectrum of the modified CUE correlation kernel is given by $p_{k}^{N}=\Psi\left(\frac{|k|-N}{\tau N^{\alpha}}\right)$, (1.36). Moreover, to keep the notation simple, we will write $p_{k}$ instead of $p_{k}^{N}$. We begin by proving a classical CLT at the Poisson scales $(\delta<\alpha)$. The proof relies on an elementary variance computation. Observe that the asymptotic variance of Theorem 2.1 matches that of Theorem 1.4 only up to a multiplicative constant. The difference is due to our normalization. Namely, the scaling (1.36) implies that the modified CUEs have density $2 \mathrm{~N}$ at the origin and

$$
\mathbb{E}_{K_{p}^{N}}\left[\Xi f_{\delta}\right]=\left\{2 N+\underset{N \rightarrow \infty}{O}\left(N^{\alpha}\right)\right\} N^{-\delta} \int f(x) d x .
$$

THEOREM 2.1. Consider a modified CUE with kernel (1.35)-(1.36) and let $f \in H_{0}^{1 / 2}(\mathbb{R})$. For any scale $0 \leq \delta<\alpha<1$, the centered and rescaled linear statistic $N^{\frac{\delta-\alpha}{2}} \tilde{\Xi} f_{\delta}$ converges in distribution to a Gaussian random variable with variance $2 \tau\|f\|_{L^{2}(\mathbb{R})}^{2}$. 
PROOF. When $n=2$, equation (2.2) reads

$$
C_{K_{p}^{N}}^{2}\left[\Xi f_{\delta}\right]=N^{-2 \delta} \sum_{u \in \mathbb{Z}} \hat{f}\left(u N^{-\delta}\right) \hat{f}\left(-u N^{-\delta}\right) \sum_{k \in \mathbb{Z}} p_{k}\left(1-p_{k+u}\right) .
$$

We let $\sigma_{k}^{2}=p_{k}\left(1-p_{k}\right)$ for any $k \geq 0$. Since $p_{-k}=p_{k}$, we have for any $u \in \mathbb{Z}$,

$$
\begin{aligned}
\sum_{k \in \mathbb{Z}} p_{k}\left(1-p_{k+u}\right) & =p_{0}\left(1-p_{u}\right)+\sum_{k>0} p_{k}\left(2-p_{k+u}-p_{k-u}\right) \\
& =p_{0}\left(1-p_{u}\right)+2 \sum_{k>0} \sigma_{k}^{2}+\sum_{k>0} p_{k}\left(2 p_{k}-p_{k+u}-p_{k-u}\right) .
\end{aligned}
$$

Since the coefficients $p_{k} \in[0,1]$ and the shape $\Psi$ is nonincreasing, we can check that for any $u \in \mathbb{Z}$,

$$
\begin{aligned}
& \left|\sum_{k>0} p_{k}\left(p_{k}-p_{k+u}\right)\right| \leq|u| \\
& \quad \text { so that }\left|\sum_{k \in \mathbb{Z}} p_{k}\left(1-p_{k+u}\right)-2 \sum_{k>0} \sigma_{k}^{2}\right| \leq 2|u|+1 .
\end{aligned}
$$

If we combine this estimate with formula (2.4), for any real-valued test function $f$, we obtain

$$
\begin{gathered}
\left.\left|N^{\delta} \mathrm{C}_{K_{p}^{N}}^{2}\left[\Xi f_{\delta}\right]-2 N^{-\delta} \sum_{u \in \mathbb{Z}}\right| \hat{f}\left(u N^{-\delta}\right)\right|^{2} \sum_{k>0} \sigma_{k}^{2} \mid \\
\leq N^{-\delta} \sum_{u \in \mathbb{Z}}\left|\hat{f}\left(u N^{-\delta}\right)\right|^{2}(2|u|+1) .
\end{gathered}
$$

If we also assume that $f \in H^{1 / 2}(\mathbb{R})$, the RHS satisfies

$$
N^{-\delta} \sum_{u \in \mathbb{Z}}\left|\hat{f}\left(u N^{-\delta}\right)\right|^{2}(2|u|+1) \leq C N^{\delta} \int_{0}^{\infty}|\hat{f}(v)|^{2}|v| d v
$$

and (2.6) yields the estimate

$$
N^{\delta-\alpha} C_{K_{p}^{N}}^{2}\left[\Xi f_{\delta}\right]=2 N^{-\delta} \sum_{u \in \mathbb{Z}}\left|\hat{f}\left(u N^{-\delta}\right)\right|^{2} N^{-\alpha} \sum_{k>0} \sigma_{k}^{2}+\underset{N \rightarrow \infty}{O}\left(N^{\delta-\alpha}\|f\|_{H^{1 / 2}}^{2}\right) .
$$

Moreover, according to formula (1.6), a Riemann sum approximation gives

$$
\lim _{N \rightarrow \infty} N^{-\alpha} \sum_{k>0} \sigma_{k}^{2}=\int_{\mathbb{R}} \Psi\left(t \tau^{-1}\right)\left(1-\Psi\left(t \tau^{-1}\right)\right) d t=\tau \mathrm{B}_{\Psi}^{2} .
$$

By convention $\mathrm{B}_{\Psi}^{2}=1$ and we conclude that when $\delta<\alpha$,

$$
\lim _{N \rightarrow \infty} N^{\delta-\alpha} C_{K_{p}^{N}}^{2}\left[\Xi f_{\delta}\right]=2 \tau \int_{-\infty}^{\infty}|\hat{f}(\xi)|^{2} d \xi
$$


Since the variance of the random variable $\Xi f_{\delta}$ is diverging like $N^{\alpha-\delta}$ and its expected value is of order $N^{1-\delta}$ by formula (2.3), the CLT follows from Soshnikov's theorem 1 in [40].

Note that, using the upper-bound (2.5) and the limit (2.7), we have for all $u \in \mathbb{Z}$

$$
\sum_{k \in \mathbb{Z}} p_{k}\left(1-p_{k+u}\right) \leq 2|u|+\underset{N \rightarrow \infty}{O}\left(N^{\alpha}\right) .
$$

Hence, by formula (2.4),

$$
C_{K_{p}^{N}}^{2}\left[\Xi f_{\delta}\right] \leq 2 N^{-\delta} \sum_{u \in \mathbb{Z}}\left|\hat{f}\left(u N^{-\delta}\right)\right|^{2}\left\{\left|u N^{-\delta}\right|+\underset{N \rightarrow \infty}{O}\left(N^{\alpha-\delta}\right)\right\} .
$$

This shows that for any $f \in H_{0}^{1 / 2}$, the variance of the linear statistic $\Xi f_{\delta}$ remains bounded in the regime $\delta \geq \alpha$. Actually, if (1.36) holds, we have in the regime $\delta>\alpha$,

$$
\lim _{N \rightarrow \infty} \operatorname{Var}_{K_{p}^{N}}\left[\Xi f_{\delta}\right]=\|f\|_{H^{1 / 2}}^{2}
$$

This suggests that at any scale $\delta>\alpha$, we should observe the same limit theorem for the modified CUEs as for the mesoscopic CUE and sine process. We can prove formula (2.9) in the same way we obtained Theorem 2.1 but the argument is already technical and becomes really involved when it comes to computing the limits of higher-order cumulants. A better approach consists in deducing the CLT from Soshnikov's theorem [39] by proving that the cumulants of a given linear statistic have the same limits regardless of the shape $\Psi$ of the modified CUE.

THEOREM 2.2. Consider a modified CUE with kernel (1.35)-(1.36) and let $f \in H_{0}^{1}(\mathbb{R})$. For any scale $1 \geq \delta>\alpha>0$, the linear statistics $\Xi f_{\delta}$ converges in distribution to a Gaussian random variable with variance $\|f\|_{H^{1 / 2}}^{2}$.

PROOF. Let us decompose $p_{k}=\mathbb{1}_{|k| \leq N}+\varepsilon_{k}$ where $\varepsilon_{k}=\Psi\left(\frac{|k|-N}{N^{\alpha}}\right)-\mathbb{1}_{|k| \leq N}$. We can write

$$
\mathrm{C}_{K_{p}^{N}}^{n}\left[\Xi f_{\delta}\right]=\mathrm{C}_{K^{N}}^{n}\left[\Xi f_{\delta}\right]+\mathcal{E}_{N}^{n}(f, \delta, \alpha, \Psi)
$$

and $\mathcal{E}_{N}^{n}$ collects all the terms which contain at least one factor $\varepsilon_{k+u_{1}+\cdots+u_{\bar{m}_{i}}}$ when we expand the products $\prod_{i=0}^{l-1} p_{k+u_{1}+\cdots+u_{\bar{m}_{i}}}$ in formula (2.2). Plainly, all other terms exactly add up to $C_{K^{N}}^{n}\left[\Xi f_{\delta}\right]$ and, since $\left|p_{k}\right|,\left|\varepsilon_{k}\right| \leq 1$ for all $k \in \mathbb{Z}$, we have

$$
\left|\mathcal{E}^{N}(f, \delta, \alpha, \Psi)\right| \leq 2 \sum_{l=1}^{n} \frac{1}{l} \sum_{\mathbf{m} \in \mathbb{N}^{l}}\left(\begin{array}{c}
n \\
\mathbf{m}
\end{array}\right) N^{-n \delta} \sum_{u \in \mathbb{Z}_{0}^{n}}\left|\prod_{i} \hat{f}\left(u_{i} N^{-\delta}\right)\right| \sum_{k \geq 0}\left|\varepsilon_{k}\right| .
$$


Moreover, by the definition of $\varepsilon_{k}$, we have the estimates

$$
\begin{aligned}
\sum_{0 \leq k \leq N}\left|\varepsilon_{k}\right| & =\sum_{-N \leq k \leq 0} 1-\Psi\left(k N^{-\alpha}\right) \leq C N^{\alpha} \int_{-\infty}^{0} 1-\Psi(t) d t, \\
\sum_{N<k}\left|\varepsilon_{k}\right| & =\sum_{0 \leq k} \Psi\left(k N^{-\alpha}\right) \leq C N^{\alpha} \int_{0}^{\infty} \Psi(t) d t .
\end{aligned}
$$

Both integrals are finite since $\Psi \in \mathfrak{F}$ and there exists a positive constant $C_{n}>0$ such that

$$
\left|\mathcal{E}^{N}(f, \delta, \alpha, \Psi)\right| \leq C_{n} N^{\alpha-\delta} \int_{\mathbb{R}_{0}^{n}} \prod_{i}\left|\hat{f}\left(v_{i}\right)\right| d^{n-1} v
$$

The assumption $f \in H_{0}^{1}(\mathbb{R})$, guarantees that for any $n \in \mathbb{N}$,

$$
\int_{\mathbb{R}_{0}^{n}} \prod_{i}\left|\hat{f}\left(v_{i}\right)\right| d^{n-1} v<\infty,
$$

so that $\mathcal{E}^{N}=O\left(N^{\alpha-\delta}\right)$ as $N \rightarrow \infty$. Therefore, the cumulants $C_{K_{p}^{N}}^{n}\left[\Xi f_{\delta}\right]$ and $C_{K^{N}}^{n}\left[\Xi f_{\delta}\right]$ have the same limits for all $n \in \mathbb{N}$ and the CLT follows directly from Theorem 1 in [39].

REMARK 2.3. In the terminology of Definition 1.10, we have just proved that the rescaled kernels $N^{-\delta} K_{p}^{N}\left(N^{-\delta} x, N^{-\delta} y\right)$ and $N^{-\delta} K^{N}\left(N^{-\delta} x, N^{-\delta} y\right)$ are asymptotically equivalent in the regime $\delta>\alpha$. We could also have deduced this fact from Lemma 2.6 below by checking that the CUE kernel $K^{N}$ which is given by (1.37) satisfies the property $L^{1} B$ at any scale $\delta \in[0,1]$.

2.3. The critical regime. It remains to look at what happens at the critical scale $\delta=\alpha$. We have already seen that the variance remains bounded as $N \rightarrow \infty$ and we can compute its limit by applying a Riemann sum approximation to formula (2.4). By symmetry,

$$
\mathrm{C}_{K_{p}^{N}}^{2}\left[\Xi f_{\alpha}\right] \simeq 2 N^{-2 \alpha} \sum_{u>0} \hat{f}\left(u N^{-\alpha}\right) \hat{f}\left(-u N^{-\alpha}\right) \sum_{k>0}\left(p_{k-u}+p_{k+u}\right)\left(1-p_{k}\right) .
$$

Since $p_{N+j}=\Psi\left(\frac{j}{\tau N^{\alpha}}\right)$ for any $j>-N$, we can check that for any $0<\alpha<1$ and for any $\tau>0$,

$$
\lim _{N \rightarrow \infty} \operatorname{Var}_{K_{p}^{N}}\left[\Xi f_{\alpha}\right]
$$

$$
\begin{aligned}
& =2 \int_{0}^{\infty} \hat{f}(u) \hat{f}(-u) \int_{\mathbb{R}}\left(\Psi\left(\frac{t-u}{\tau}\right)+\Psi\left(\frac{t+u}{\tau}\right)\right)\left(1-\Psi\left(\frac{t}{\tau}\right)\right) d t d u \\
& =2 \int_{\mathbb{R}}|\hat{f}(u)|^{2} \int_{\mathbb{R}} \Psi\left(\frac{t+u}{\tau}\right)\left(1-\Psi\left(\frac{t}{\tau}\right)\right) d t d u .
\end{aligned}
$$


Because of some subtle cancellations, it is difficult to use formula (2.2) to compute the limits of the higher-order cumulants by Riemann sum approximations. Another approach is to rewrite the correlation kernel of the modified CUE before using the cumulants formulae. From Definition 1.12, a summation by parts yields

$$
\begin{aligned}
K_{p}^{N}(x, y) & =\sum_{k=0}^{\infty}\left(p_{k}^{N}-p_{k+1}^{N}\right) K^{k}(x, y) \\
& =\frac{1}{\tau N^{\alpha}} \sum_{k=-N}^{\infty} \Phi\left(\frac{k+\xi_{k}}{\tau N^{\alpha}}\right) \frac{\sin ((2 N+2 k+1) \pi(x-y))}{\sin (\pi(x-y))}
\end{aligned}
$$

where the parameters $\xi_{k} \in(0,1)$ are given by the mean-value theorem. Now, we may use formula (2.12) to relate the kernel $K_{p}^{N}$ to the sine kernel.

Proposition 2.4. At the critical scale $\delta=\alpha$, the modified CUE kernel $K_{p}^{N}$ and the kernel $L_{\Psi, \eta}^{N}$ given by (1.33) with $\eta(k)=\left(N+k+\frac{1}{2}\right) N^{-\alpha}$ are asymptotically equivalent in the sense of Definition 1.10.

By Proposition 1.11, a similar approximation holds for the modified GUEs. There is only a minor difference in the definition of the function $\eta$ so that the limits of the cumulants of both models can be computed in a common framework which is presented in Section 4. In order to prove Propositions 1.11 and 2.4, we need to provide a criterion to check whether two kernels are asymptotically equivalent. First, we need to introduce a new definition. A similar concept was introduced in [32] to control cumulants of some complex determinantal processes.

Definition 2.5. A family of kernels $\left(L^{N}\right)_{N>0}$ satisfies the property $L^{1} B$ if for any compact set $A \subseteq \mathfrak{X}$, there exists a sequence $\Gamma_{N}: \mathfrak{X} \rightarrow \mathbb{R}^{+}$and $v>0$ such that and all $(x, y) \in A^{2}$,

$$
\left|L^{N}(x, y)\right| \leq \Gamma_{N}(x-y)
$$

and

$$
\left\|\Gamma_{N}\right\|_{L^{1}(\tilde{A})}=\underset{N \rightarrow \infty}{O}\left(|\log N|^{\nu}\right)
$$

where $\tilde{A}=\{x=y-z: y, z \in A\}$.

LEMMA 2.6. Two families of kernels $\left(L^{N}\right)_{N>0}$ and $\left(K^{N}\right)_{N>0}$ are asymptotically equivalent if $\left(L^{N}\right)_{N>0}$ has the property $L^{1} B$ and there exists $\kappa>0$ such that for any compact set $A \subseteq \mathfrak{X}$,

$$
\sup \left\{\left|L^{N}(x, y)-K^{N}(x, y)\right|:(x, y) \in A^{2}\right\}=\underset{N \rightarrow \infty}{O}\left(N^{-\kappa}\right) .
$$


Proof. Let $\ell \in \mathbb{N}$ and $f_{1}, \ldots, f_{\ell} \in C_{0}(\mathbb{R})$. If we replace $K^{N}=L^{N}+E^{N}$, we get

$$
\operatorname{Tr}\left[K^{N} f_{1} \cdots K^{N} f_{\ell}\right]=\operatorname{Tr}\left[L^{N} f_{1} \cdots L^{N} f_{\ell}\right]+\sum_{J^{k} \in\left\{L^{N}, E^{N}\right\}} \operatorname{Tr}\left[J^{1} f_{1} \cdots J^{\ell} f_{\ell}\right] .
$$

Note that all terms of the last sum contains at least one operator $E^{N}$. By assumption, we can suppose that all the test functions are supported in a compact set $A$ and $\sup \left\{\left|E^{N}(x, y)\right|:(x, y) \in A^{2}\right\} \leq C N^{-\kappa}$. If we first look at a trace which contains a single operator $E^{N}$, by formula (1.17), we have the estimate:

$$
\begin{aligned}
& \left|\operatorname{Tr}\left[J^{1} f_{1} \cdots J^{\ell} f_{\ell}\right]\right| \\
& \quad \leq C N^{-\kappa} \prod_{k=2}^{\ell}\left\|f_{k}\right\|_{\infty} \int_{A^{\ell}}\left|f_{1}\left(x_{1}\right) L^{N}\left(x_{1}, x_{2}\right) \cdots L^{N}\left(x_{\ell-1}, x_{\ell}\right)\right| \prod_{j} d \mu\left(x_{j}\right) .
\end{aligned}
$$

Since $L^{N}$ has the property $L^{1} B$, there exists $\Gamma_{N}: \mathfrak{X} \rightarrow \mathbb{R}^{+}$such that $\left|L^{N}\left(x_{k}, x_{k+1}\right)\right| \leq \Gamma_{N}\left(x_{k}-x_{k+1}\right)$ and a change of variables yields

$$
\left|\operatorname{Tr}\left[J^{1} f_{1} \ldots J^{l} f_{\ell}\right]\right| \leq C N^{-\kappa} \prod_{k=2}^{\ell}\left\|f_{k}\right\|_{\infty}\left\|\Gamma_{N}\right\|_{L^{1}(\tilde{A})}^{\ell-1}\left\|f_{1}\right\|_{L^{1}}
$$

A similar argument shows that any trace which contains $j$ operators $E^{N}$ is bounded by $N^{-j \kappa}$ times a logarithmic correction coming from $\left\|\Gamma_{N}\right\|_{L^{1}(\tilde{A})}$. Therefore, using the notation $\bar{O}$ introduced in Section 1.5, we get

$$
\operatorname{Tr}\left[K^{N} f_{1} \cdots K^{N} f_{\ell}\right]=\operatorname{Tr}\left[L^{N} f_{1} \cdots L^{N} f_{\ell}\right]+\underset{N \rightarrow \infty}{\bar{O}}\left(N^{-\kappa}\right) .
$$

According to Definition 1.10, this completes the proof.

ProOf OF Proposition 2.4. A Taylor expansion of the function $\sin (\pi(x-$ $y) N^{-\alpha}$ ) in the denominator of formula (2.12) shows that

$$
\begin{aligned}
& N^{-\alpha} K_{p}^{N}\left(x N^{-\alpha}, y N^{-\alpha}\right) \\
& \quad=\frac{1}{\tau N^{\alpha}} \sum_{k=-N}^{\infty} \Phi\left(\frac{k+\xi_{k}}{\tau N^{\alpha}}\right) \frac{\sin (2 \pi \eta(k)(x-y))}{\pi(x-y)}+\underset{N \rightarrow \infty}{O}\left(N^{-\alpha}\right)
\end{aligned}
$$

and the error term is uniform over any compact subset of $\mathbb{R}^{2}$. Then, by Lemma 2.6, it is enough to prove that the RHS family of kernels which we denoted by $L_{\Psi, \eta}^{N}(x, y)$ satisfies the property $L^{1} B$. Since the kernel $L_{\Psi, \eta}^{N}(x, y)$ is translationinvariant on $\mathbb{R}$, we can choose $\Gamma_{N}(x)=\left|L_{\Psi, \eta}^{N}(0, x)\right|$. It is well known that there 
exists a universal constant $C>0$ such that for any $s>0$ and $n>0$,

$$
\int_{-s}^{s}\left|\frac{\sin n(x-y)}{x-y}\right| d y \leq C \log (s n) .
$$

This implies that

$$
\left\|\Gamma_{N}\right\|_{L^{1}[-s, s]} \leq \frac{C}{\tau N^{\alpha}} \sum_{k=-N}^{\infty} \Phi\left(\frac{k+\xi_{k}}{\tau N^{\alpha}}\right) \log (s \eta(k)) .
$$

Since $\eta(k)=N^{1-\alpha}+\frac{k+1 / 2}{N^{\alpha}}$ and $\int_{0}^{\infty} \Phi(t) \log t d t<\infty$, we deduce from the estimate (2.15) that there is a constant $C$ which only depends on the shape $\Psi$ so that $\left\|\Gamma_{N}\right\|_{L^{1}[-s, s]} \leq C \log (s N)$.

Proposition 2.4 implies that the determinantal processes with correlation kernels $K_{p}^{N}$ and $L_{\Psi, \eta}^{N}$ have the same limit at the critical scale. By Corollary 4.10, this yields the following limit theorem for linear statistics of the critical modified CUEs.

THEOREM 2.7. Let $f \in H_{0}^{1}(\mathbb{R}), 0<\alpha<1$ and $\Psi \in \mathfrak{E}$. The linear statistic $\Xi f_{\alpha}$ of the determinantal process with correlation kernel (1.35)-(1.36) converges in distribution as $N \rightarrow \infty$ to a random variable $\Xi_{\Psi, \tau} f$ whose cumulants are given by formula (1.31). In particular, up to a scaling, this is the same limit theorem as for the critical modified GUEs.

3. Central limit theorems for the modified GUEs. We begin in Section 3.1 by proving some technical lemmas that are required to compute the asymptotic variance of linear statistics of the modified GUEs. In Section 3.2, we prove Theorem 1.5 by comparing the rescaled correlation kernel of a modified GUE to that of the classical GUE using the perturbative method developed in Section 2.3. All these results are based on the asymptotics of the Hermite polynomials and the GUE kernel which are presented in Appendix A.

3.1. Proof of Theorem 1.4. We start by proving a classical formula for the variance of linear statistics which is valid in a general context.

LEMMA 3.1. Given a determinantal process with a correlation kernel $K$ of type (1.3), for any test function $f \in C_{0}(\mathfrak{X})$, we have

$$
\begin{aligned}
\operatorname{Var}_{K}[\Xi f]= & \sum_{k=0}^{\infty} \sigma_{k}^{2} \int f(x)^{2}\left|\varphi_{k}(x)\right|^{2} \mu(d x) \\
& +\frac{1}{2} \iint(f(x)-f(y))^{2}|K(x, y)|^{2} \mu(d x) \mu(d y),
\end{aligned}
$$

where $\sigma_{k}^{2}=p_{k}^{N}\left(1-p_{k}^{N}\right)$. 
PROOF. If we apply formula (1.19) when $n=2$,

$$
\begin{aligned}
\operatorname{Var}_{K}[\Xi f]= & \int f(x)^{2} K(x, x) \mu(d x) \\
& -\iint f(x) f(y) K(x, y) K(y, x) \mu(d x) \mu(d y) \\
= & \frac{1}{2} \iint(f(x)-f(y))^{2} K(x, y) K(y, x) \mu(d x) \mu(d y) \\
& -\iint f(x)^{2} K(x, y) K(y, x) \mu(d x) \mu(d y) \\
& +\int f(x)^{2} K(x, x) \mu(d x) .
\end{aligned}
$$

Note that when the kernel $K$ is reproducing, the last two terms cancel. In general, since the function $\psi_{k}$ are orthonormal, we get

$$
\begin{aligned}
-\iint & f(x)^{2} K(x, y) K(y, x) \mu(d x) \mu(d y)+\int f(x)^{2} K(x, x) \mu(d x) \\
= & -\sum_{k, j} p_{k} p_{j} \int \varphi_{j}(y) \overline{\varphi_{k}}(y) \mu(d y) \int f(x)^{2} \varphi_{k}(x) \overline{\varphi_{j}}(x) \mu(d x) \\
& +\sum_{k} p_{k} \int f(x)^{2} \varphi_{k}(x) \overline{\varphi_{k}}(x) \mu(d x) \\
= & \sum_{k} \sigma_{k}^{2} \int f(x)^{2}\left|\varphi_{k}(x)\right|^{2} \mu(d x) .
\end{aligned}
$$

We can use Lemma 3.1 to compute the asymptotics of the variance for linear statistics of the modified GUEs. We call the reproducing variance the quantity

$$
V_{0}(f)=\frac{1}{2} \iint|f(x)-f(y)|^{2}\left|K_{\Psi, \alpha}^{N}(x, y)\right|^{2} d x d y .
$$

This definition comes from the fact that, if the correlation kernel $K$ is reproducing, then $\sigma_{k}^{2}=0$ for all $k \in \mathbb{Z}_{+}$and $\operatorname{Var}_{K}[\Xi f]=V_{0}(f)$ for any linear statistic. On the other hand, we call the Poisson variance the quantity

$$
V_{\sigma}(f)=\sum_{k=0}^{\infty} \sigma_{k}^{2} \int f(x)^{2}\left|\varphi_{k}(x)\right|^{2} d x .
$$

This is motivated by the observation that $\operatorname{Var}_{K}[\#]=V_{\sigma}(1)$ and, as we already mentioned in the Introduction, this quantity measures the extra randomness induced in the process because the correlation kernel is not reproducing. In particular, for a 
modified GUE we have

$$
\sigma_{k}^{2}=\Psi\left(\frac{k-N}{\tau N^{\alpha}}\right)\left(1-\Psi\left(\frac{k-N}{\tau N^{\alpha}}\right)\right)
$$

and

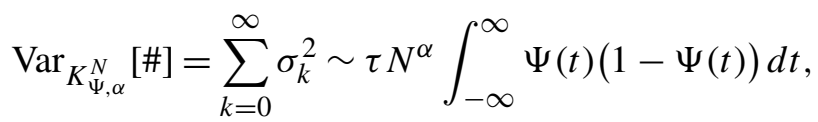

which gives formula (1.9). We shall see that, except at the critical scale $\delta=\alpha$, there is only one component of the variance which is asymptotically relevant.

LEMMA 3.2. For any $0<\alpha, \delta<1$ and for any $f \in C_{0}(\mathbb{R})$, we have

$$
V_{\sigma}\left(f_{\delta}\right)=\frac{\tau}{2} N^{\alpha-\delta} \int_{\mathbb{R}} f(x)^{2} d x+\underset{N \rightarrow \infty}{o}\left(N^{\alpha-\delta}\right) .
$$

REMARK 3.3. It is not difficult to adapt the proof so that Lemma 3.2 holds for any function $f \in L^{2}(\mathbb{R})$ which is uniformly continuous. In particular, by Morrey's inequality, this covers all test functions in the Sobolev space $H^{1}(\mathbb{R})$.

PROOF. Since the rescaled Hermite functions satisfy $\left\|\varphi_{k}\right\|_{L^{2}}=1$ for all $k \geq 0$ and we suppose that the test function $f$ is bounded, by formula (3.2), for any $0<\epsilon<1$,

$$
V_{\sigma}\left(f_{\delta}\right)=\sum_{|k|<N^{1-\epsilon}} \sigma_{N+k}^{2} \int f_{\delta}(x)^{2}\left|\varphi_{N+k}(x)\right|^{2} d x+O\left(\|f\|_{\infty}^{2} \sum_{|k|>N^{1-\epsilon}} \sigma_{N+k}^{2}\right) .
$$

The condition $\Psi \in \mathfrak{F}$ guarantees that, if $\epsilon<1-\alpha$, the error term is converging to 0 as $N \rightarrow \infty$. Actually, under the stronger assumption that $\Psi \in \mathfrak{E}$, this term decays faster than any power of $N$ and it will be neglected in the following. Moreover the assumption that $f$ has compact support in conjunction with the condition $|k|<N^{1-\epsilon}$ implies that we can use the bulk asymptotic for the Hermite functions, formula (A.2). Namely, for any $x \in \operatorname{supp}(f)$,

$$
\varphi_{N+k}\left(x N^{-\delta}\right)=\cos \left[(N+k)\left(\frac{\pi}{2}-F\left(x_{N} N^{-\delta}\right)\right)\right]+\underset{n \rightarrow \infty}{O}\left(N^{-\delta}\right),
$$

where we set $x_{N}=x \frac{\pi}{2} \sqrt{\frac{N}{N+k}}$. Then

$$
\begin{aligned}
V_{\sigma}\left(f_{\delta}\right)= & N^{-\delta} \sum_{|k|<N^{1-\epsilon}} \sigma_{N+k}^{2} \\
& \times\left\{\int f(x)^{2}\left|\cos \left[(N+k)\left(\frac{\pi}{2}-F\left(x_{N} N^{-\delta}\right)\right)\right]\right|^{2} d x+O\left(N^{-\delta}\right)\right\} .
\end{aligned}
$$


Observe that according to formula (3.4), we have $\sum_{|k|<N^{1-\epsilon}} \sigma_{N+k}^{2}=O\left(N^{\alpha}\right)$ and the previous estimate gives

$$
\begin{aligned}
V_{\sigma}\left(f_{\delta}\right)= & \frac{N^{-\delta}}{2}\left\{\operatorname{Var}_{K_{\Psi, \alpha}^{N}}[\#]\|f\|_{L^{2}}^{2}\right. \\
& +\sum_{|k|<N^{1-\epsilon}}(-1)^{N+k} \sigma_{N+k}^{2} \\
& \left.\times \int f(x)^{2} \cos \left[2(N+k) F\left(x_{N} N^{-\delta}\right)\right] d x+O\left(N^{\alpha-\delta}\right)\right\} .
\end{aligned}
$$

The second term is a sum of oscillatory integrals and we will show that it converges to 0 . Let us make the change of variable $z=N^{\delta} F\left(x_{N} N^{-\delta}\right)$. By Definition A.1,

$$
\begin{aligned}
\int f(x)^{2} & \cos \left[2(N+k) F\left(x_{N} N^{-\delta}\right)\right] d x \\
= & \frac{2}{\pi} \sqrt{1+\frac{k}{N}} \\
& \times \int f^{2}\left(\frac{2 N^{\delta}}{\pi} \sqrt{1+\frac{k}{N}} G\left(z N^{-\delta}\right)\right) G^{\prime}\left(z N^{-\delta}\right) \cos \left[2(N+k) N^{-\delta} z\right] d z .
\end{aligned}
$$

Since the function $f$ is uniformly continuous (we assume that $f$ has compact support in $\left.\left[-\frac{L}{2}, \frac{L}{2}\right]\right)$, there exists a sequence $\epsilon_{N} \searrow 0$ such that uniformly over all $|z|<L$ and all $|k|<N^{1-\epsilon}$,

$$
\left|f^{2}\left(\frac{2 N^{\delta}}{\pi} \sqrt{1+\frac{k}{N}} G\left(z N^{-\delta}\right)\right)-f^{2}\left(\frac{2 G^{\prime}(0) z}{\pi}\right)\right| \leq \epsilon_{N} .
$$

Since $G^{\prime}(0)=1 / 2$, it follows that for any $|k|<N^{1-\epsilon}$,

$$
\begin{aligned}
& \int f(x)^{2} \cos \left[2(N+k) F\left(x_{N} N^{-\delta}\right)\right] d x \\
& \quad=\frac{1}{\pi} \int f^{2}\left(\frac{z}{\pi}\right) \cos \left[2(N+k) N^{-\delta} z\right] d z+O\left(\epsilon_{N}\right) .
\end{aligned}
$$

Since the sequence $\left(N-N^{1-\epsilon}\right) N^{-\delta} \rightarrow \infty$ as $N \rightarrow \infty$, by the Riemann-Lebesgue lemma, we can also assume that

$$
\sup _{|k| \leq N^{1-\epsilon}}\left|\int f(x)^{2} \cos \left[2(N+k) F\left(x_{N} N^{-\delta}\right)\right] d x\right| \leq \epsilon_{N} .
$$

Thus, going back to the Poisson variance, we have shown that

$$
V_{\sigma}\left(f_{\delta}\right)=\frac{N^{-\delta}}{2} \operatorname{Var}_{K_{\Psi, \alpha}^{N}}[\#]\left\{\|f\|_{L^{2}}^{2}+\underset{N \rightarrow \infty}{O}\left(\epsilon_{N}\right)\right\} .
$$


The lemma follows after replacing $\operatorname{Var}_{K_{\Psi, \alpha}^{N}}[\#]$ by formula (1.9).

In order to prove formula (1.22), it remains to estimate the reproducing variance $V_{0}(f)$. Before proceeding, we need to recall a few properties of the GUE correlation kernel (1.4). We refer to Appendix A for further details. First, note that according to the convention of Definition 1.1, the Christoffel-Darboux formula, (A.6), implies that for any $x, y \in \mathbb{R}$ and $M \geq 0$,

$$
(x-y) K_{0}^{M}(x, y)=\sqrt{M / N}\left(\varphi_{M}(x) \varphi_{M-1}(y)-\varphi_{M-1}(x) \varphi_{M}(y)\right) .
$$

Moreover, the uniform bound (A.5) for the Hermite functions implies that there exists $C>0$ such that, for any $n \geq 0$,

$$
\left\|\varphi_{n}\right\|_{\infty} \leq C N^{1 / 4} n^{-1 / 12} .
$$

In particular, $\left|K_{0}^{M}(x, y)\right| \leq C \sqrt{N} M$ and by formula (3.6), this gives us a simple bound for the GUE kernel. For any $x, y \in \mathbb{R}$, we have

$$
\left|K_{0}^{M}(x, y)\right| \leq C(\sqrt{N} M) \wedge \frac{M^{1 / 3}}{|x-y|} .
$$

The connection with the modified GUE kernel comes from a summation by parts:

$$
\begin{aligned}
K_{\Psi, \alpha}^{N}(x, y) & =\sum_{k=0}^{\infty}\left(p_{k}-p_{k+1}\right) K_{0}^{k}(x, y) \\
& =\frac{1}{\tau N^{\alpha}} \sum_{k=-N}^{\infty} \Phi\left(\frac{k+\xi_{k}}{\tau N^{\alpha}}\right) K_{0}^{N+k}(x, y),
\end{aligned}
$$

where $\Phi=-\Psi^{\prime}$ and $\xi_{k} \in(0,1)$ are given by the mean-value theorem. If we further suppose that the shape $\Psi \in \mathfrak{E},(1.11)$, there exists c $>0$ so that for any $\Gamma>0$,

$$
\left|\frac{1}{\tau N^{\alpha}} \sum_{|k|>\Gamma N^{\alpha}} \Phi\left(\frac{k+\xi_{k}}{\tau N^{\alpha}}\right) K_{0}^{N+k}(x, y)\right| \leq C e^{-\mathrm{c} \Gamma}\left(N^{3 / 2} \wedge \frac{N^{1 / 3}}{|x-y|}\right) .
$$

By formula (3.8), this implies that for any sequence $\Gamma_{N}>0$,

$$
K_{\Psi, \alpha}^{N}(x, y)=\frac{1}{\tau N^{\alpha}} \sum_{|k| \leq \Gamma_{N} N^{\alpha}} \Phi\left(\frac{k+\xi_{k}}{\tau N^{\alpha}}\right) K_{0}^{N+k}(x, y)
$$

$$
+\underset{N \rightarrow \infty}{O}\left(e^{-\mathrm{c} \Gamma_{N}}\left(N^{3 / 2} \wedge \frac{N^{1 / 3}}{|x-y|}\right)\right)
$$

uniformly for all $x, y \in \mathbb{R}$. 
REMARK 3.4. The assumption $\Psi \in \mathfrak{E}$ implies that by choosing $\Gamma_{N}=$ $(\log N)^{2}$, the error in formula (3.10) decays faster than any power of $N$. Analogous approximations hold for more general shapes, although with an error term which may not be good enough to deal with all mesoscopic scales. Moreover, the condition $\Psi \in \mathfrak{E}$ leads to a straightforward proof, otherwise we would need to take into account the speed of decay of $\Psi$ and to produce more precise estimates.

LEMMA 3.5. For any $0<\alpha<1$ and any scale $0<\delta \leq 1$, there exists a constant $C>0$ such that for any function $f \in H_{0}^{1 / 2}(\mathbb{R})$, the reproducing variance satisfies for all sufficiently large $N$,

$$
V_{0}\left(f_{\delta}\right) \leq C\|f\|_{H^{1 / 2}}^{2} .
$$

Proof. To simplify the notation, let us assume that the temperature $\tau=1$ and set the parameters $\xi_{k}=0$. We will also let $x_{N}=x N^{-\delta}, y_{N}=y N^{-\delta}$ and fix $L>0$ so that $\operatorname{supp}(f) \subset\left[-\frac{L}{2}, \frac{L}{2}\right]$. By formula (3.1) and the approximation (3.10),

$$
\begin{aligned}
V_{0}\left(f_{\delta}\right)= & \frac{1}{2} \iint\left|f_{\delta}(x)-f_{\delta}(y)\right|^{2}\left|K_{\Psi, \alpha}^{N}(x, y)\right|^{2} d x d y \\
\leq & \iint\left|f_{\delta}(x)-f_{\delta}(y)\right|^{2}\left|\widetilde{K}_{\Psi, \alpha}^{N}(x, y)\right|^{2} d x d y \\
& +\underset{N \rightarrow \infty}{O}\left(\left\|f_{\delta}\right\|_{H^{1 / 2}}^{2} N^{1 / 3} e^{-c \Gamma_{N}}\right),
\end{aligned}
$$

where

$$
\widetilde{K}_{\Psi, \alpha}^{N}(x, y)=N^{-\alpha} \sum_{|k| \leq \Gamma_{N} N^{\alpha}} \Phi\left(k N^{-\alpha}\right) K_{0}^{N+k}(x, y) .
$$

By formula (1.20), $\left\|f_{\delta}\right\|_{H^{1 / 2}}^{2}=\|f\|_{H^{1 / 2}}^{2}$ and, if we let $\Gamma_{N}=(\log N)^{2}$, the error in the previous estimate converges to 0 and it remains to show that

$$
\begin{aligned}
& \iint\left|f_{\delta}(x)-f_{\delta}(y)\right|^{2}\left|\widetilde{K}_{\Psi, \alpha}^{N}(x, y)\right|^{2} d x d y \\
& \quad=\iint|f(x)-f(y)|^{2}\left|N^{-\delta} \widetilde{K}_{\Psi, \alpha}^{N}\left(x_{N}, y_{N}\right)\right|^{2} d x d y \\
& \quad \leq C\|f\|_{H^{1 / 2}}^{2} .
\end{aligned}
$$

According to the sine-kernel approximation (A.10), if the density $N$ is sufficiently large compared to $L$, there exists a constant $C>0$ such that for all $|k| \leq \Gamma_{N} N^{\alpha}$ and for all $x, y \in[-L, L]$,

$$
\left|N^{-\delta} K_{0}^{N+k}\left(x_{N}, y_{N}\right)\right| \leq \frac{C}{|x-y|} .
$$


Since, for any $\Gamma>0$,

$$
\frac{1}{\tau N^{\alpha}} \sum_{|k| \leq \Gamma N^{\alpha}} \Phi\left(\frac{k+\xi_{k}}{\tau N^{\alpha}}\right) \leq 1,
$$

this implies that for all $x, y \in[-L, L]$,

$$
\left|N^{-\delta} \widetilde{K}_{\Psi, \alpha}^{N}\left(x_{N}, y_{N}\right)\right| \leq \frac{C}{|x-y|} .
$$

Hence, by a change of variables,

$$
\begin{aligned}
& \iint\left|f_{\delta}(x)-f_{\delta}(y)\right|^{2}\left|\widetilde{K}_{\Psi, \alpha}^{N}(x, y)\right|^{2} d x d y \\
& \leq C \iint_{[-L, L]^{2}}\left|\frac{f(x)-f(y)}{x-y}\right|^{2} d x d y \\
& \quad+\iint_{\mathbb{R}^{2} \backslash[-L, L]}|f(x)-f(y)|^{2}\left|N^{-\delta} \widetilde{K}_{\Psi, \alpha}^{N}\left(x_{N}, y_{N}\right)\right|^{2} d x d y .
\end{aligned}
$$

For any $L>0$, the first integral on the RHS of (3.15) is bounded by $\|f\|_{H^{1 / 2}}^{2}$ and to obtain the upper-bound (3.13), it suffices to show that there exists a constant $C(f) \geq 0$ which only depends on the test function $f$ such that

$$
\iint_{\mathbb{R}^{2} \backslash[-L, L]}|f(x)-f(y)|^{2}\left|N^{-\delta} \widetilde{K}_{\Psi, \alpha}^{N}\left(x_{N}, y_{N}\right)\right|^{2} d x d y \leq \frac{C(f)}{L} .
$$

Thus, choosing the parameter $L$ sufficiently large, by formula (3.11), this implies that the variance $V_{0}\left(f_{\delta}\right) \leq C\|f\|_{H^{1 / 2}}^{2}$. The rest of the proof is rather technical and is devoted to prove the estimate (3.16). Since the function $f$ is supported in $\left[-\frac{L}{2}, \frac{L}{2}\right]$, by symmetry of the GUE kernel, we have

$$
\begin{aligned}
& \iint_{\mathbb{R}^{2} \backslash[-L, L]^{2}}|f(x)-f(y)|^{2}\left|N^{-\delta} \widetilde{K}_{\Psi, \alpha}^{N}\left(x_{N}, y_{N}\right)\right|^{2} d x d y \\
& \leq 4 N^{-2 \alpha} \sum_{\substack{|k| \leq \Gamma N^{\alpha} \\
|j| \leq \Gamma N^{\alpha}}} \Phi\left(k N^{-\alpha}\right) \Phi\left(j N^{-\alpha}\right) \iint_{\substack{|y|<L / 2 \\
x>L}}\left|\frac{f(x)-f(y)}{x-y}\right|^{2} \\
& \quad \times\left|\left(x_{N}-y_{N}\right)^{2} K_{0}^{N+k}\left(x_{N}, y_{N}\right) K_{0}^{N+j}\left(x_{N}, y_{N}\right)\right| d x d y .
\end{aligned}
$$

Since $\varphi_{k}(x)=\sqrt{\frac{\pi \sqrt{N}}{\sqrt{2}}} h_{k}\left(x \frac{\pi \sqrt{N}}{\sqrt{2}}\right)$, we deduce from the bulk asymptotic (A.2) with $\gamma=1 / 6$ that there exists a universal constant $C>0$ such that for all $|k| \leq \Gamma_{N} N^{\alpha}$ and for all $|x|<\frac{2(\sqrt{N+k}-1)}{\pi \sqrt{N}}$,

$$
\left|\varphi_{N+k}(x)\right| \leq \frac{C}{\left(4 \frac{N+k}{N}-\pi^{2} x^{2}\right)^{1 / 4}} .
$$


In particular, for any $|y|<L / 2$, we have $\left|\varphi_{N+k}\left(y_{N}\right)\right| \leq C$, and by formula (3.6), we get

$$
\begin{aligned}
\mid\left(x_{N}-\right. & \left.y_{N}\right)^{2} K_{0}^{N+k}\left(x_{N}, y_{N}\right) K_{0}^{N+j}\left(x_{N}, y_{N}\right) \mid \\
\leq & C^{2}\left(\left|\varphi_{N+k}\left(x_{N}\right) \varphi_{N+j}\left(x_{N}\right)\right|+\left|\varphi_{N+k}\left(x_{N}\right) \varphi_{N+j-1}\left(x_{N}\right)\right|\right. \\
& \left.\quad+\left|\varphi_{N+k-1}\left(x_{N}\right) \varphi_{N+j}\left(x_{N}\right)\right|+\left|\varphi_{N+k-1}\left(x_{N}\right) \varphi_{N+j-1}\left(x_{N}\right)\right|\right) .
\end{aligned}
$$

Let

$$
J_{k, j}=\iint_{\substack{|y|<L / 2 \\ x>L}}\left|\frac{f(x)-f(y)}{x-y}\right|^{2}\left|\varphi_{N+k}\left(x_{N}\right) \varphi_{N+j}\left(x_{N}\right)\right| d x d y .
$$

By (3.17), we see that there exists $C>0$ such that

$$
\begin{gathered}
\iint_{\mathbb{R}^{2} \backslash[-L, L]^{2}}|f(x)-f(y)|^{2}\left|N^{-\delta} \widetilde{K}_{\Psi, \alpha}^{N}\left(x_{N}, y_{N}\right)\right|^{2} d x d y \\
\leq C N^{-2 \alpha} \sum_{\substack{|k| \leq \Gamma N^{\alpha} \\
|j| \leq \Gamma N^{\alpha}}} \Phi\left(k N^{-\alpha}\right) \Phi\left(j N^{-\alpha}\right) \\
\times\left\{J_{k, j}+J_{k, j-1}+J_{k-1, j}+J_{k-1, j-1}\right\} .
\end{gathered}
$$

So that in order to prove the estimate (3.16), by (3.14), it remains to show that $J_{k, j} \leq C(f) / L$ for all $|k|,|j| \leq \Gamma N^{\alpha}$. To do so, we shall combine the asymptotics from Appendix A. First of all, since $\operatorname{supp}(f) \subset\left[-\frac{L}{2}, \frac{L}{2}\right]$, we have for any $|x|>L$,

$$
\left|\frac{f(x)-f(y)}{x-y}\right| \leq \mathbb{1}_{y \in \operatorname{supp}(f)} \frac{2\|f\|_{\infty}}{|x-L / 2|} \leq \mathbb{1}_{y \in \operatorname{supp}(f)} \frac{4\|f\|_{\infty}}{|x|}
$$

and, if $C(f)=4\|f\|_{\infty}^{2}|\operatorname{supp} f|$, we get

$$
\int_{-L / 2}^{L / 2}\left|\frac{f(x)-f(y)}{x-y}\right|^{2} d y \leq \frac{C(f)}{|x|^{2}} .
$$

Hence, by a change of variables,

$$
J_{k, j} \leq C(f) N^{-\delta} \int_{L N^{-\delta}}^{\infty}\left|\varphi_{N+k}(x) \varphi_{N+j}(x)\right| \frac{d x}{x^{2}} .
$$

Suppose that $j \geq k$ and let $a_{ \pm}=\frac{2(\sqrt{N+k} \pm 1)}{\pi \sqrt{N}}$. We split the integral:

$$
\begin{aligned}
\int_{L N^{-\delta}}^{\infty}\left|\varphi_{N+k}(x) \varphi_{N+j}(x)\right| \frac{d x}{x^{2}} \\
=\left\{\int_{L N^{-\delta}}^{a_{-}}+\int_{a_{-}}^{a_{+}}+\int_{a_{+}}^{\infty}\right\}\left|\varphi_{N+k}(x) \varphi_{N+j}(x)\right| \frac{d x}{x^{2}} .
\end{aligned}
$$


Using the upper-bound (3.18), the first integral is bounded by

$$
\int_{L N^{-\delta}}^{a_{-}}\left|\varphi_{N+k}(x) \varphi_{N+j}(x)\right| \frac{d x}{x^{2}} \leq C^{2} \int_{L N^{-\delta}}^{a_{-}} \frac{d x}{x^{2}\left(4 \frac{N+k}{N}-\pi^{2} x^{2}\right)^{1 / 2}} .
$$

Since $a_{-}^{2} \leq 4 \frac{N+k}{\pi N}$ when $N$ is sufficiently large, we obtain

$$
\int_{L N^{-\delta}}^{a_{-}}\left|\varphi_{N+k}(x) \varphi_{N+j}(x)\right| \frac{d x}{x^{2}} \leq \frac{C N^{\delta}}{L} .
$$

Using the uniform bound (3.7), since $a_{+}-a_{-}=\frac{4}{\pi} N^{-1 / 2}$, the contribution from the edge integral gives

$$
\int_{a_{-}}^{a_{+}}\left|\varphi_{N+k}(x) \varphi_{N+j}(x)\right| \frac{d x}{x^{2}} \leq C N^{-1 / 6} .
$$

Finally, we have

$$
\int_{a_{+}}^{\infty}\left|\varphi_{N+k}(x) \varphi_{N+j}(x)\right| \frac{d x}{x^{2}} \leq C N^{1 / 6} \int_{a_{+}}^{\infty}\left|\varphi_{N+k}(x)\right| \frac{d x}{x^{2}},
$$

and the estimate (A.4) implies that, for all $x>a_{+}$,

$$
\left|\varphi_{N+k}(x)\right| \leq C N^{1 / 4} e^{-\frac{2 \sqrt{2} \pi N^{3 / 4}}{3 \sqrt{N+k}}} .
$$

Thus, we obtain for all $|k|,|j| \leq \Gamma N^{\alpha}$,

$$
\int_{a_{+}}^{\infty}\left|\varphi_{N+k}(x) \varphi_{N+j}(x)\right| \frac{d x}{x^{2}} \leq C N^{5 / 12} e^{-2 \sqrt{2} N^{1 / 4}} .
$$

If we put together the estimates (3.22)-(3.24), we have proved that when $N$ is sufficiently large,

$$
\int_{L N^{-\delta}}^{\infty}\left|\varphi_{N+k}(x) \varphi_{N+j}(x)\right| \frac{d x}{x^{2}} \leq \frac{C N^{\delta}}{L} .
$$

Hence, it follows from formula (3.20) that for any $|j|,|k| \leq \Gamma_{N} N^{\alpha}$, the integral $J_{k, j} \leq C(f) / L$. By (3.19) and (3.14), we conclude that the estimate (3.16) holds and this completes the proof.

We are now ready to give the proof of formula (1.22), and hence of Theorem 1.4. It follows immediately from Lemmas 3.1, 3.2 and 3.5 that in the regime $\delta<\alpha$, for any test function $f \in H_{0}^{1 / 2} \cap L^{\infty}(\mathbb{R})$,

$$
\operatorname{Var}_{K_{\Psi, \alpha}^{N}}\left[\Xi f_{\delta}\right]=\frac{\tau}{2} N^{\alpha-\delta} \int_{\mathbb{R}} f(x)^{2} d x+\underset{N \rightarrow \infty}{o}\left(N^{\alpha-\delta}\right) .
$$

Note that the same argument shows that, in the regime $\delta \geq \alpha$,

$$
\operatorname{Var}_{K_{\Psi, \alpha}^{N}}\left[\Xi f_{\delta}\right] \leq C\left(\|f\|_{L^{2}}^{2}+\|f\|_{H^{1 / 2}}^{2}\right) \text {. }
$$


At the GUE scales $(\delta>\alpha)$, the limit of the variance is given by Theorem 1.5 which is proved in the next section. At the critical scale $\delta=\alpha$, by Lemma 3.2, the Poisson variance $V_{\sigma}\left(f_{\alpha}\right)$ converges to $\frac{\tau}{2}\|f\|_{L^{2}}^{2}$ and the limit of the reproducing variance $V_{0}\left(f_{\alpha}\right)$ is computed in Appendix B by a Riemann sum approximation.

\subsection{Proof of Theorem 1.5.}

THEOREM 3.6. Let $\Xi$ be the GUE eigenvalue process with correlation kernel $K_{0}^{N}$ given by (1.4). For any $0<\delta<1$ and any $f \in H_{0}^{1 / 2} \cap L^{\infty}(\mathbb{R})$, we have as the number of eigenvalues $N \rightarrow \infty$,

$$
\widetilde{\Xi} f_{\delta} \Rightarrow \mathcal{N}\left(0,\|f\|_{H^{1 / 2}}^{2}\right)
$$

Theorem 3.6 was first established in [6, 7] for the resolvent function $x \mapsto$ $(x-z)^{-1}$ where $\Im z>0$. A general proof was given only recently in [19]. Their argument exploits a nice connection between the characteristic polynomial of a GUE matrix and a log-correlated Gaussian process. In [5], a generalization of Theorem 3.6 is proved for Gaussian $\beta$-Ensembles. Yet another generalization to certain classes of orthogonal polynomial ensembles is made in $[9,25]$. In particular, the proof of Theorem 3.6 in [25] is based on the cumulant computations presented in Section 4 and the sine-kernel asymptotics of Theorem A.2. We now turn to the approximation of the modified GUE correlation kernels at the so-called GUE scales $(\delta>\alpha)$. By Definition 1.10, Proposition 3.7 below combined with Theorem 3.6 implies the central limit Theorem 1.5.

Proposition 3.7. For any shape $\Psi \in \mathfrak{E}$, the kernel $K_{\Psi, \alpha}^{N}$ and the GUE kernel $K_{0}^{N}$ are asymptotically equivalent at any scale $\delta>\alpha$,

$$
N^{-\delta} K_{\Psi, \alpha}^{N}\left(N^{-\delta} x, N^{-\delta} y\right) \cong N^{-\delta} K_{0}^{N}\left(N^{-\delta} x, N^{-\delta} y\right) .
$$

ProOF. To simplify the notation, let us assume that the temperature $\tau=1$ and the parameters $\xi_{k}=0$. The condition $\Psi \in \mathfrak{E}$ implies that for any $\Gamma>0$,

$$
\sum_{k>\Gamma N^{\alpha}} \Psi\left(k N^{-\alpha}\right)+\sum_{k<-\Gamma N^{\alpha}}\left(1-\Psi\left(k N^{-\alpha}\right)\right) \leq C N^{\alpha} e^{-\Gamma} .
$$

So, if we let $\Gamma_{N}=(\log N)^{2}$, these sums decay faster than any power of $N$ and combined with the uniform bound (3.7), this implies that

$$
\begin{aligned}
K_{\Psi, \alpha}^{N}(x, y) \simeq & \sum_{k=0}^{N-1} \varphi_{k}(x) \varphi_{k}(y)+\sum_{k=0}^{\Gamma_{N} N^{\alpha}} \Psi\left(k N^{-\alpha}\right) \varphi_{N+k}(x) \varphi_{N+k}(y) \\
& -\sum_{k=1}^{\Gamma_{N} N^{\alpha}}\left(1-\Psi\left(-k N^{-\alpha}\right)\right) \varphi_{N-k}(x) \varphi_{N-k}(y)
\end{aligned}
$$


with a uniform error. Moreover, for any $L>0$, the bulk estimate (3.18) implies that that all $x, y \in[-L, L]$,

$$
\sum_{|k| \leq \Gamma_{N} N^{\alpha}}\left|\varphi_{N+k}\left(N^{-\delta} x\right) \varphi_{N+k}\left(N^{-\delta} y\right)\right| \leq 2 C^{2} \Gamma_{N} N^{\alpha}
$$

Since the function $\Psi \in[0,1]$, this yields

$$
N^{-\delta} K_{\Psi, \alpha}^{N}\left(N^{-\delta} x, N^{-\delta} y\right)=N^{-\delta} \sum_{k=0}^{N-1} \varphi_{k}\left(N^{-\delta} x\right) \varphi_{k}\left(N^{-\delta} y\right)+\underset{N \rightarrow \infty}{\bar{O}}\left(N^{\alpha-\delta}\right)
$$

uniformly for all $x, y \in[-L, L]$. The sum on the RHS corresponds to the rescaled GUE kernel. Thus, by Lemma 2.6, to prove that the kernels $K_{\Psi, \alpha}^{N}$ and $K_{0}^{N}$ are asymptotically equivalent, it remains to show that the latter satisfies the property $L^{1} B$. Taking $M=N$ in the approximation (A.10) implies that there exists a positive constant $C_{L}$ such that for any $0<\delta \leq 1$,

$$
\left|N^{-\delta} K_{0}^{N}\left(x N^{-\delta}, y N^{-\delta}\right)\right| \leq \Gamma_{N}(x-y):=C_{L} \begin{cases}N & \text { if }|x-y|<\frac{\log N}{N} \\ \frac{1}{|x-y|} & \text { if }|x-y| \geq \frac{\log N}{N}\end{cases}
$$

and we immediately check that $\int_{-L}^{L} \Gamma_{N}(z) d z \leq 4 C_{L} \log N$.

4. Cumulants of the critical models. In this section we prove Theorems 1.7 and 2.7 , then we analyze the random processes $\Xi_{\Psi, \tau}$ which arise from the critical modified ensembles. In fact, we will not investigate directly the modified ensembles but the processes with kernel $L_{\Psi, \eta}^{N}$ given by (1.33). By Propositions 1.11 and 2.4, there are two choices of the function $\eta$ which correspond to the modified GUEs and CUEs, respectively. However, our analysis works as long as $\eta$ satisfies the conditions (4.4)-(4.5) below. In Section 4.1, we show that $L_{\Psi, \eta}^{N}$ is the correlation kernel of a determinantal process and we prove Proposition 1.11. The convergence of smooth linear statistics of these processes is established in Section 4.2; see Corollary 4.10. The main result in Section 4.3 is that, for all $\Psi \in \mathfrak{F}$ such that $\Psi \neq \psi$ and for all $\tau>0$, the random variables $\Xi_{\Psi, \tau} f$ are not Gaussian. Then, in Section 4.4 , we show by constructing an example that, despite the special property of the shape $\psi$, the MNS ensemble at the critical scale also converges to a random process which is not Gaussian.

\subsection{Asymptotically equivalent kernels for the critical modified GUEs.}

LEMMA 4.1. Let $N, \tau, \Gamma>0, \alpha \in(0,1), \Psi \in \mathfrak{F}$ and $\eta$ be a nondecreasing function. The kernel $L_{\Psi, \eta}^{N}$ given by (1.33) defines a translation-invariant determinantal process on $\mathbb{R}$. 
PROOF. Since the kernel $L_{\Psi, \eta}^{N}$, we can define its Fourier transform:

$$
\widehat{L}_{\Psi, \eta}^{N}(v)=\frac{1}{\tau N^{\alpha}} \sum_{|k| \leq \Gamma N^{\alpha}} \Phi\left(\frac{k+\xi_{k}}{\tau N^{\alpha}}\right) \mathbb{1}_{[-\eta(k), \eta(k)]}(v) .
$$

Plainly, the function $\widehat{L}_{\Phi, \eta}^{N} \in L^{1}(\mathbb{R})$ and by (1.33),

$$
L_{\Psi, \eta}^{N}(x, y)=\int_{\mathbb{R}} \widehat{L}_{\Psi, \eta}^{N}(v) e^{i 2 \pi v(x-y)} d v .
$$

In [40], it is established that for any translation-invariant kernel $L$, the condition $0 \leq \widehat{L} \leq 1$, guarantees that it defines a determinantal point process. Recall that the parameters $\xi_{k}$ have been chosen so that $\frac{1}{\tau N^{\alpha}} \Phi\left(\frac{k+\xi_{k}}{\tau N^{\alpha}}\right)=\Psi\left(\frac{k}{\tau N^{\alpha}}\right)-\Psi\left(\frac{k+1}{\tau N^{\alpha}}\right)$ and it follows that for any $v \in \mathbb{R}$,

$$
\widehat{L}_{\Psi, \eta}^{N}(v) \leq \Psi\left(-\Gamma \tau^{-1}\right) \leq 1
$$

Moreover, since $\Phi \geq 0$ by assumption, $\widehat{L}_{\Psi, \eta}^{N} \geq 0$ and we conclude that $L_{\Psi, \eta}^{N}$ is the correlation kernel of a determinantal process.

Proof of Proposition 1.11. Let $1 / 3<\alpha<1, \Psi \in \mathfrak{E}$, and $\Gamma_{N}=(\log N)^{2}$. We also assume that $\tau=1$ and combine the approximation (3.10) of the modified GUE kernel $K_{\Psi, \alpha}^{N}$ with the asymptotic formula of Theorem A.3 with $x_{0}=0$. Namely, taking $\delta=\alpha$ in formula (A.11), we obtain

$$
\begin{aligned}
& N^{-\alpha} K_{\Psi, \alpha}^{N}\left(x N^{-\alpha}, y N^{-\alpha}\right) \\
& \quad=N^{-\alpha} \sum_{|k| \leq \Gamma_{N} N^{\alpha}} \Phi\left(\frac{k+\xi_{k}}{N^{\alpha}}\right) \frac{\sin \left[\pi N^{1-\alpha} \sqrt{1+k / N}(x-y)\right]}{\pi(x-y)}+O\left(N^{1-3 \alpha}\right) .
\end{aligned}
$$

The estimates (2.15) shows that, with $\eta(k)=\frac{1}{2} N^{1-\alpha} \sqrt{1+k / N}$, the kernel $L_{\Psi, \eta}^{N}$ given by (1.33) has the property $L^{1} B$ and it follows from Lemma 2.6 that

$$
N^{-\alpha} K_{\Psi, \alpha}^{N}\left(x N^{-\alpha}, y N^{-\alpha}\right) \cong L_{\Psi, \eta}^{N}(x, y) .
$$

Propositions 1.11 and 2.4 imply that each of the modified ensembles have an asymptotically equivalent kernel at the critical scale of the form $L_{\Psi, \eta}^{N}$ with

(i) $\eta(k)=(N+k) N^{-\alpha}$ for the modified CUEs,

(ii) $\eta(k)=\frac{1}{2} N^{1-\alpha} \sqrt{1+k / N}$ for the modified GUEs.

In the sequel, we will compute the limits of the cumulants for any determinantal process with kernel $L_{\Psi, \eta}^{N}$ which satisfies the following conditions. The function $\eta$ is nondecreasing and it satisfies uniformly for all $|k| \leq \Gamma_{N} N^{\alpha}$,

$$
\eta(k)=N^{\nu}+\beta k N^{-\alpha}+\underset{N \rightarrow \infty}{O}\left(N^{-\epsilon}\right),
$$


where $\nu, \beta, \epsilon>0$ such that $N^{v} \gg \Gamma_{N}$ and

$$
\lim _{N \rightarrow \infty} N^{v} \max \left\{\Psi\left(\Gamma_{N}\right), 1-\Psi\left(-\Gamma_{N}\right)\right\}=0 .
$$

In particular, for the modified GUEs (resp., CUEs), the asymptotics (4.4) holds with $\nu=1-\alpha$ and $\beta=1 / 4$ (resp., $\beta=1$ ) and, if the shape $\Psi \in \mathfrak{E}$, the condition (4.5) holds for any $\alpha \in(0,1)$ with $\Gamma_{N}=(\log N)^{2}$.

4.2. Proof of Theorem 1.7. Given the expression (4.2) for the kernel $L_{\Psi, \eta}^{N}$, we can repeat the proof of Lemma 1.14 replacing sums by integrals and we get the following formula:

$$
\begin{aligned}
C_{L_{\Psi, \eta}^{N}}^{n}[\Xi f]= & \int_{\mathbb{R}_{0}^{n}} d^{n-1} u \prod_{i} \hat{f}\left(u_{i}\right) \sum_{|\mathbf{m}|=n} \mathrm{M}(\mathbf{m}) \\
& \times \int_{\mathbb{R}} \prod_{i=1}^{\ell(\mathbf{m})} \widehat{L}_{\Phi, \eta}^{N}\left(v+u_{1}+\cdots+u_{\bar{m}_{i}}\right) d v,
\end{aligned}
$$

where the sum is over all compositions $\mathbf{m}$ of $n \in \mathbb{N},(1.18)$. Combining this formula with (4.1), we get an expression for the cumulants that is appropriate to pass to the limit as $N \rightarrow \infty$. In this section, to simplify the notation, we will assume that $\xi_{k}=0$ and (unless stated otherwise) all sums run over $\left|k_{i}\right| \leq \Gamma_{N} N^{\alpha}$. We define for any composition $\mathbf{m}$ of $n \geq 2$, the function

$$
\mathrm{H}_{\mathbf{m}}(u, k)=\int_{\mathbb{R}}^{\ell(\mathbf{m})} \prod_{i=1} \mathbb{1}_{\left|v+u_{1}+\cdots+u_{\bar{m}_{i}}\right| \leq \eta\left(k_{i}\right)} d v .
$$

LEMMA 4.2. For any $f \in C_{0}(\mathbb{R})$, the cumulants of a linear statistics of the determinantal process with correlation kernel (1.33) are given by

$$
\begin{aligned}
C_{L_{\Psi, \eta}^{N}}^{n}[\Xi f] \simeq & \left(\frac{1}{\tau N^{\alpha}}\right)^{n} \sum_{k_{1} \leq \cdots \leq k_{n}} \prod_{i=1}^{n} \Phi\left(\frac{k_{i}}{\tau N^{\alpha}}\right) \\
& \times \int_{\mathbb{R}_{0}^{n}} \prod_{i} \hat{f}\left(u_{i}\right) \sum_{|\mathbf{m}|=\mathbf{n}} \mathrm{M}(\mathbf{m}) \sum_{\sigma \in \mathbb{S}(n)} \mathrm{H}_{\mathbf{m}}(u, \sigma k) d^{n-1} u .
\end{aligned}
$$

Proof. To simplify the notation, let us also assume that $\tau=1$. By formula (4.1), for any composition $\mathbf{m}$ of $n$ of length $\ell$ and any $v \in \mathbb{R}^{\ell}$, we have

$$
\prod_{i=1}^{\ell} \hat{L}_{\Phi, \eta}^{N}\left(v_{i}\right)=N^{-\alpha \ell} \sum_{k_{1}, \ldots, k_{\ell}} \prod_{i=1}^{\ell} \Phi\left(\frac{k_{i}}{N^{\alpha}}\right) \mathbb{1}_{\left|v_{i}\right| \leq \eta\left(k_{i}\right)} .
$$


If we let $\epsilon_{N}^{n}=0$ and $\epsilon_{N}^{\ell}=1-N^{-\alpha(n-\ell)} \sum_{k_{\ell+1}, \ldots, k_{n}} \prod_{i=\ell+1}^{n} \Phi\left(\frac{k_{i}}{N^{\alpha}}\right)$ for all $\ell \leq n$, we get

$$
\prod_{i=1}^{\ell} \hat{L}_{\Phi, \eta}^{N}\left(v_{i}\right)\left\{1-\epsilon_{N}^{\ell}\right\}=N^{-\alpha n} \sum_{k_{1}, \ldots, k_{n}} \prod_{i=1}^{n} \Phi\left(\frac{k_{i}}{N^{\alpha}}\right) \prod_{i=1}^{\ell} \mathbb{1}_{\left|v_{i}\right| \leq \eta\left(k_{i}\right)} .
$$

By (4.7), this implies that

$$
\begin{gathered}
\left\{1-\epsilon_{N}^{\ell}\right\} \int_{\mathbb{R}} \prod_{i=1}^{\ell} \hat{L}_{\Phi, \eta}^{N}\left(v+u_{1}+\cdots+u_{\bar{m}_{i}}\right) d v \\
=N^{-\alpha n} \sum_{k_{1}, \ldots, k_{n}} \prod_{i=1}^{n} \Phi\left(\frac{k_{i}}{N^{\alpha}}\right) \mathrm{H}_{\mathbf{m}}(u, k) .
\end{gathered}
$$

Observe that by (4.9), since $N^{-\alpha} \sum_{\kappa} \Phi\left(\kappa N^{-\alpha}\right) \leq 1$ and we assume that the condition (4.4) holds, we have $\int_{\mathbb{R}} \prod_{i=1}^{\ell} \hat{L}_{\Phi, \eta}^{N}\left(v_{i}\right) d v_{1} \leq C N^{v}$. Moreover, by definition $0 \leq \epsilon_{N}^{\ell} \leq \Psi(\Gamma)$, so that according to condition (4.5),

$$
\lim _{N \rightarrow \infty} \epsilon_{N}^{\ell} \int_{\mathbb{R}} \prod_{i=1}^{\ell} \hat{L}_{\Phi, \eta}^{N}\left(v+u_{1}+\cdots+u_{\bar{m}_{i}}\right) d v=0 .
$$

Thus, by formula (4.10),

$$
\begin{aligned}
& \int_{\mathbb{R}} \prod_{i=1}^{\ell} \hat{L}_{\Phi, \eta}^{N}\left(v+u_{1}+\cdots+u_{\bar{m}_{i}}\right) d v \\
& \quad \simeq N^{-\alpha n} \sum_{k_{1} \leq \cdots \leq k_{n}} \prod_{i=1}^{n} \Phi\left(\frac{k_{i}}{N^{\alpha}}\right) \sum_{\sigma \in \mathbb{S}(n)} \mathrm{H}_{\mathbf{m}}(u, \sigma k) .
\end{aligned}
$$

We conclude by using formula (4.6).

In the following, we will always use the conventions $\ell=\ell(\mathbf{m}), \Lambda_{i, s}^{\mathbf{m}}=\Lambda_{i, s}^{\mathbf{m}}(u)$ and $\mathfrak{s}=\mathfrak{s}_{\ell(\mathbf{m})}(\sigma)$. Moreover, can express the function $\mathrm{H}_{\mathbf{m}}(u, k)$ using the notation (1.26)-(1.27).

LEMMA 4.3. Let $k \in \mathbb{Z}_{\leq}^{n}$ and $u \in \mathbb{R}_{0}^{n}$. For any permutation $\sigma \in \mathbb{S}(n)$ and any composition $\mathbf{m}$ of $n \geq 2$,

$$
\begin{aligned}
\mathrm{H}_{\mathbf{m}}(u, \sigma k)= & {\left[2 \eta\left(k_{\sigma(\mathfrak{s})}\right)-\max _{i \leq \ell}\left\{\Lambda_{i, \mathfrak{s}}^{\mathbf{m}}-\eta\left(k_{\sigma(i)}\right)+\eta\left(k_{\sigma(\mathfrak{s})}\right)\right\}\right.} \\
& \left.-\max _{i \leq \ell}\left\{-\Lambda_{i, \mathfrak{s}}^{\mathbf{m}}-\eta\left(k_{\sigma(i)}\right)+\eta\left(k_{\sigma(\mathfrak{s})}\right)\right\}\right]^{+} .
\end{aligned}
$$


PROOF. Let $v_{i}=\sum_{j=1}^{\bar{m}_{i}} u_{j}$. The change of variable $w=v-v_{\mathfrak{s}}$ in (4.7) gives

$$
\mathrm{H}_{\mathbf{m}}(u, \sigma k)=\int_{\mathbb{R}} \prod_{i=1}^{\ell} \mathbb{1}_{\left|w+\Lambda_{i, \mathfrak{s}}^{\mathbf{m}}\right| \leq \eta\left(k_{\sigma(i)}\right)} d w=\left|\bigcap_{i=1}^{\ell}\left\{w:\left|w+\Lambda_{i, \mathfrak{s}}^{\mathbf{m}}\right| \leq \eta\left(k_{\sigma(i)}\right)\right\}\right| .
$$

By definition $\min _{i \leq \ell}\{\sigma(i)\}=\sigma(\mathfrak{s})$, and since the function $\eta$ is nondecreasing, for any $k \in \mathbb{Z}_{\leq}^{n}$,

$$
\eta\left(k_{\sigma(\mathfrak{s})}\right)=\min _{i \leq \ell} \eta\left(k_{\sigma(i)}\right) .
$$

Then, since $\Lambda_{\mathfrak{s , \mathfrak { s }}}^{\mathbf{m}}=0$ by (1.26), we obtain

$$
\begin{aligned}
\bigcap_{i=1}^{\ell}\left\{\left|w+\Lambda_{i, \mathfrak{s}}^{\mathbf{m}}\right| \leq \eta\left(k_{\sigma(i)}\right)\right\}= & {\left[-\eta\left(k_{\sigma(\mathfrak{s})}\right)+\max _{i \leq l}\left\{-\Lambda_{i, \mathfrak{s}}^{\mathbf{m}}-\eta\left(k_{\sigma(i)}\right)+\eta\left(k_{\sigma(\mathfrak{s})}\right)\right\},\right.} \\
& \left.\eta\left(k_{\sigma(\mathfrak{s})}\right)-\max _{i \leq l}\left\{\Lambda_{i, \mathfrak{s}}^{\mathbf{m}}-\eta\left(k_{\sigma(i)}\right)+\eta\left(k_{\sigma(\mathfrak{s})}\right)\right\}\right] .
\end{aligned}
$$

This interval is nonempty if the condition

$$
\begin{aligned}
2 \eta\left(k_{\sigma(\mathfrak{s})}\right)> & \max _{i \leq \ell}\left\{\Lambda_{i, \mathfrak{s}}^{\mathbf{m}}-\eta\left(k_{\sigma(i)}\right)+\eta\left(k_{\sigma(\mathfrak{s})}\right)\right\} \\
& +\max _{i \leq \ell}\left\{-\Lambda_{i, \mathfrak{s}}^{\mathbf{m}}-\eta\left(k_{\sigma(i)}\right)+\eta\left(k_{\sigma(\mathfrak{s})}\right)\right\}
\end{aligned}
$$

is satisfied, which completes the proof.

We are now ready to prove our main result, that is, to compute the limits of the cumulants of linear statistics of the modified ensembles by applying a Riemann sum approximation to formula (4.8). The argument is quite similar to the proof of Lemma 2 in [39], but more involved. To keep the proof as transparent as possible, it relies on three lemmas which will be proved afterwards.

THEOREM 4.4. Assume that the conditions (4.4)-(4.5) are satisfied and let $f \in H_{0}^{1}(\mathbb{R})$. For any $n \geq 2$,

$$
\begin{aligned}
\lim _{N \rightarrow \infty} \mathrm{C}_{L_{\Psi, \eta}^{N}}^{n}[\Xi f] \\
=2 \beta \tau \mathrm{B}_{\Psi}^{n} \int_{\mathbb{R}} f(t)^{n} d t \\
\quad-2 \int_{\mathbb{R}_{0}^{n}} d u \int_{\mathbb{R}_{<}^{n}} d x \Re\left\{\prod_{i=1}^{n} \hat{f}\left(u_{i}\right) \Phi\left(x_{i}\right)\right\} \sum_{|\mathbf{m}|=n} \mathbf{M}(\mathbf{m}) \mathrm{G}_{\beta \tau}^{\mathbf{m}}(u, x),
\end{aligned}
$$

where $\mathrm{G}_{\tau}^{\mathbf{m}}(u, x)$ and $\mathrm{B}_{\Psi}^{n}$ are defined by formulae (1.28) and (1.29). 
PROOF. Throughout the proof, we will use the familiar inequality (3.14) without any reference. Let $|u|_{1}=\left|u_{1}\right|+\cdots+\left|u_{n}\right|$ and

$$
\begin{aligned}
\Upsilon_{N}^{n}(u)= & \left(\frac{1}{\tau N^{\alpha}}\right)^{n} \sum_{k_{1} \leq \cdots \leq k_{n}} \prod_{i=1}^{n} \Phi\left(\frac{k_{i}}{\tau N^{\alpha}}\right) \\
& \times \sum_{|\mathbf{m}|=n} \mathbf{M}(\mathbf{m}) \\
& \times \sum_{\sigma \in \mathbb{S}(n)}\left(\eta\left(k_{\sigma(\mathfrak{s})}\right)-\max _{i \leq \ell}\left\{\Lambda_{i, \mathfrak{s}}^{\mathbf{m}}-\eta\left(k_{\sigma(i)}\right)+\eta\left(k_{\sigma(\mathfrak{s})}\right)\right\}\right) .
\end{aligned}
$$

If the parameter $N$ is sufficiently large, we claim that for any $u \in \mathbb{R}_{0}^{n}$, any $\sigma \in \mathbb{S}(n)$, and for all $k \in \mathbb{Z}_{\leq}^{n}$ such that $|k|_{\infty}<\Gamma_{N} N^{\alpha}$,

$$
\begin{aligned}
& \mid \mathrm{H}_{\mathbf{m}}(u, \sigma k)-2 \eta\left(k_{\sigma(\mathfrak{s})}\right)+\max _{i \leq \ell}\left\{\Lambda_{i, \mathfrak{s}}^{\mathbf{m}}-\eta\left(k_{\sigma(i)}\right)+\eta\left(k_{\sigma(\mathfrak{s})}\right)\right\} \\
& +\max _{i \leq \ell}\left\{-\Lambda_{i, \mathfrak{s}}^{\mathbf{m}}-\eta\left(k_{\sigma(i)}\right)+\eta\left(k_{\sigma(\mathfrak{s})}\right)\right\} \mid \\
& \quad \leq \begin{cases}0 & \text { if }|u|_{1} \leq \frac{N^{v}}{2}, \\
18|u|_{1} & \text { else. }\end{cases}
\end{aligned}
$$

First, note that, since $\Lambda_{\mathfrak{s}, \mathfrak{s}}^{\mathbf{m}}=0, \max _{i \leq \ell}\left\{ \pm \Lambda_{i, \mathfrak{s}}^{\mathbf{m}}-\eta\left(k_{\sigma(i)}\right)+\eta\left(k_{\sigma(\mathfrak{s})}\right)\right\} \geq 0$. Moreover, by (4.11),

$$
\max _{i \leq \ell}\left\{ \pm \Lambda_{i, \mathfrak{s}}^{\mathbf{m}}-\eta\left(k_{\sigma(i)}\right)+\eta\left(k_{\sigma(\mathfrak{s})}\right)\right\} \leq \max _{i \leq \ell}\left\{ \pm \Lambda_{i, \mathfrak{s}}^{\mathbf{m}}\right\}
$$

and, by (1.26), for any composition $\mathbf{m}$ of $n$, we have $\left|\Lambda_{i, \mathfrak{s}}^{\mathbf{m}}\right| \leq|u|_{1}$ for all $i \leq \ell$. Hence, we conclude that

$$
0 \leq \max _{i \leq \ell}\left\{ \pm \Lambda_{i, \mathfrak{s}}^{\mathbf{m}}-\eta\left(k_{\sigma(i)}\right)+\eta\left(k_{\sigma(\mathfrak{s})}\right)\right\} \leq|u|_{1} .
$$

When the parameter $N$ is large, condition (4.4) implies that for any $|\kappa| \leq \Gamma N^{\alpha}$,

$$
\frac{N^{v}}{2}<\eta(\kappa)<2 N^{v}
$$

Thus, if we also suppose that $|u|_{1} \leq \frac{N^{v}}{2}$, by (4.14),

$$
\eta\left(k_{\sigma(\mathfrak{s})}\right)>\max _{i \leq \ell}\left\{ \pm \Lambda_{i, \mathfrak{s}}^{\mathbf{m}}-\eta\left(k_{\sigma(i)}\right)+\eta\left(k_{\sigma(\mathfrak{s})}\right)\right\} .
$$

By Lemma 4.3, we conclude that when $|u|_{1} \leq \frac{N^{v}}{2}$,

$$
\begin{gathered}
\mathrm{H}_{\mathbf{m}}(u, \sigma k)-2 \eta\left(k_{\sigma(\mathfrak{s})}\right)+\max _{i \leq \ell}\left\{\Lambda_{i, \mathfrak{s}}^{\mathbf{m}}-\eta\left(k_{\sigma(i)}\right)+\eta\left(k_{\sigma(\mathfrak{s})}\right)\right\} \\
+\max _{i \leq \ell}\left\{-\Lambda_{i, \mathfrak{s}}^{\mathbf{m}}-\eta\left(k_{\sigma(i)}\right)+\eta\left(k_{\sigma(\mathfrak{s})}\right)\right\}=0 .
\end{gathered}
$$


For the second estimate, we observe that the estimate (4.15) implies that

$$
0 \leq \mathrm{H}_{\mathbf{m}}(u, \sigma k) \leq 2 \eta\left(k_{\sigma(\mathfrak{s})}\right) \leq 4 N^{\nu} .
$$

Then, by the triangle inequality and (4.14), the LHS of (4.13) is bounded by $8 N^{v}+$ $2|u|_{1}$. Thus, we have also proved (4.13) in the case when $|u|_{1}>\frac{N^{v}}{2}$. If we combine this estimate with formula (4.8) for the cumulants of the random variable $\Xi f$, there exists a positive constant $C_{n}$ which only depends on $n$ such that if the parameter $N$ is sufficiently large,

$$
\left|C_{L_{\Psi, \eta}^{N}}^{n}[\Xi f]-\int_{\mathbb{R}_{0}^{n}} \prod_{i} \hat{f}\left(u_{i}\right)\left\{\Upsilon_{N}^{n}(u)+\Upsilon_{N}^{n}(-u)\right\} d^{n-1} u\right|
$$

$$
\leq C_{n} \int_{\mathbb{R}_{0}^{n}} \mathbb{1}_{\left\{|u|_{1}>\frac{N^{v}}{2}\right\}}|u|_{1} \prod_{i}\left|\hat{f}\left(u_{i}\right)\right| d^{n-1} u,
$$

where the function $\Upsilon_{N}^{n}(u)$ is given by (4.12). Taking $f_{1}=\cdots=f_{n}=f$ in Lemma 4.6 below implies that the RHS of (4.16) converges to 0 as $N \rightarrow \infty$. Thus, the limits of the cumulants are given by

$$
\begin{aligned}
\lim _{N \rightarrow \infty} C_{L_{\Psi, \eta}^{N}}^{n}[\Xi f] & =\lim _{N \rightarrow \infty} \int_{\mathbb{R}_{0}^{n}} \prod \hat{i}\left(u_{i}\right)\left\{\Upsilon_{N}^{n}(u)+\Upsilon_{N}^{n}(-u)\right\} d^{n-1} u \\
& =2 \lim _{N \rightarrow \infty} \int_{\mathbb{R}_{0}^{n}} \Re\left\{\prod \hat{f}\left(u_{i}\right)\right\} \Upsilon_{N}^{n}(u) d^{n-1} u .
\end{aligned}
$$

The next step is to compute the limit of $\Upsilon_{N}^{n}(u)$ as $N \rightarrow \infty$; see (4.20). Observe that, according to condition (4.4) and since the max function is Lipschitz continuous, we get uniformly for all $u \in \mathbb{R}_{0}^{n}$,

$$
\begin{aligned}
\Upsilon_{N}^{n}(u)= & \left(\frac{1}{\tau N^{\alpha}}\right)^{n} \sum_{k_{1} \leq \cdots \leq k_{n}} \prod_{i=1}^{n} \Phi\left(\frac{k_{i}}{\tau N^{\alpha}}\right) \sum_{|\mathbf{m}|=n} \mathrm{M}(\mathbf{m}) \\
& \times \sum_{\sigma \in \mathbb{S}(n)}\left(\eta\left(k_{1}\right)+\beta \frac{k_{\sigma(\mathfrak{s})}-k_{1}}{N^{\alpha}}-\max _{i \leq \ell}\left\{\Lambda_{i, \mathfrak{s}}^{\mathbf{m}}-\beta \frac{k_{\sigma(i)}-k_{\sigma(\mathfrak{s})}}{N^{\alpha}}\right\}\right) \\
& +\underset{N \rightarrow \infty}{O}\left(N^{-\epsilon}\right) .
\end{aligned}
$$

By Lemma 4.5 below, $\sum \mathrm{M}(\mathbf{m})=0$ and we can remove the two terms $\eta\left(k_{1}\right)$ and $k_{1} N^{-\alpha}$ from formula (4.18) since they do not depend on $\mathbf{m}$. Hence, we have proved that

$$
\Upsilon_{N}^{n}(u)=\left(\frac{1}{\tau N^{\alpha}}\right)^{n} \sum_{k_{1} \leq \cdots \leq k_{n}} \prod_{i=1}^{n} \Phi\left(\frac{k_{i}}{\tau N^{\alpha}}\right)
$$

$$
\times \sum_{|\mathbf{m}|=n} \mathrm{M}(\mathbf{m})\left(\beta \sum_{\sigma \in \mathbb{S}(n)} \frac{k_{\sigma(\mathfrak{s})}}{N^{\alpha}}-\mathrm{G}_{\beta N^{-\alpha}}^{\mathbf{m}}(u, k)\right)+\underset{N \rightarrow \infty}{O}\left(N^{-\epsilon}\right),
$$


where $\mathrm{G}_{\beta N^{-\alpha}}^{\mathbf{m}}$ is given by (1.28). Then a Riemann sum approximation implies that

$$
\begin{aligned}
\Upsilon_{\infty}^{n}(u) & :=\lim _{N \rightarrow \infty} \Upsilon_{N}^{n}(u) \\
& =\int_{\mathbb{R}_{<}^{n}} \prod_{i=1}^{n} \Phi\left(z_{i} / \tau\right) \sum_{|\mathbf{m}|=n} \mathbf{M}(\mathbf{m})\left(\beta \sum_{\sigma \in \mathbb{S}(n)} z_{\sigma(\mathfrak{s})}-\mathrm{G}_{\beta}^{\mathbf{m}}(u, z)\right) d^{n} z
\end{aligned}
$$

The first sum is independent of the Fourier variable $u \in \mathbb{R}_{0}^{n}$ and it can be computed explicitly; see Lemma 4.7 below. Furthermore, making the change of variables $x_{i}=z_{i} \tau$, we obtain

$$
\Upsilon_{\infty}^{n}(u)=\beta \tau \mathrm{B}_{\Psi}^{n}-\int_{\mathbb{R}_{<}^{n}} \prod_{i=1}^{n} \Phi\left(x_{i}\right) \sum_{|\mathbf{m}|=n} \mathrm{M}(\mathbf{m}) \mathrm{G}_{\beta \tau}^{\mathbf{m}}(u, x) d^{n} x .
$$

Now, we can deduce the limits of the cumulants of the random variable $\Xi f$ from equations (4.17) and (4.20). By (1.28) and the estimate $\left|\Lambda_{i, \mathfrak{s}}^{\mathbf{m}}\right| \leq|u|_{1}$, we get

$$
\sup \left\{\mathrm{G}_{\tau}^{\mathbf{m}}(x, u): x \in \mathbb{R}_{>}^{n}, \tau>0\right\} \leq n !|u|_{1} .
$$

Moreover, since $\left|k_{\sigma(\mathfrak{s})}\right| \leq\left|k_{1}\right|+\left|k_{n}\right|$ for any $k \in \mathbb{Z}_{\leq}^{n}$, by formula (4.19), there exists a constant $C$ which only depends on $n$ such that for any $N>0$,

$$
\left|\Upsilon_{N}^{n}(u)\right| \leq C \int_{\mathbb{R}_{<}^{n}} \prod_{i=1}^{n} \Phi\left(x_{i} / \tau\right)\left(1+\left|x_{1}\right|+\left|x_{n}\right|+|u|_{1}\right) d^{n} x .
$$

The assumption $\Psi \in \mathfrak{F}$ guarantees that the RHS of (4.22) is finite. From Lemma 4.6 below, (4.20) and the dominated convergence theorem, we conclude that

$$
\begin{aligned}
\lim _{N \rightarrow \infty} & \mathrm{C}_{L_{\Psi, \eta}^{N}}^{N}[\Xi f] \\
= & 2 \int_{\mathbb{R}_{0}^{n}} \Re\left\{\prod_{i} \hat{f}\left(u_{i}\right)\right\} \\
& \times\left(\beta \tau \mathrm{B}_{\Psi}^{n}-\int_{\mathbb{R}_{<}^{n}} \prod_{i=1}^{n} \Phi\left(x_{i}\right) \sum_{|\mathbf{m}|=n} \mathrm{M}(\mathbf{m}) \mathrm{G}_{\beta \tau}^{\mathbf{m}}(u, x) d^{n} x\right) d^{n-1} u
\end{aligned}
$$

The final observation is that the integral over $\mathbb{R}_{0}^{n}$ can be written as a convolution, namely, a change of variables gives

$$
\begin{aligned}
\int_{\mathbb{R}_{0}^{n}} \prod_{i=1}^{n} \hat{f}\left(u_{i}\right) d^{n-1} u & =\int_{\mathbb{R}^{n-1}} \hat{f}\left(v_{1}\right) \prod_{i=2}^{n-1} \hat{f}\left(v_{i}-v_{i-1}\right) \hat{f}\left(-v_{n-1}\right) d^{n-1} v \\
& =\underbrace{\hat{f} * \cdots * \hat{f}}_{n}(0) .
\end{aligned}
$$


If we replace $\hat{f} * \cdots * \hat{f}=\widehat{f^{n}}$ and evaluate at 0 , we get $\int_{\mathbb{R}_{0}^{n}} \prod_{i=1}^{n} \hat{f}\left(u_{i}\right) d^{n-1} u=$ $\int_{\mathbb{R}} f(t)^{n} d t$ and the proof of Theorem 4.4 is complete.

Now we prove the lemmas that we used to get Theorem 4.4. The first lemma is classical, it was already used in [39], as well as in the context of other invariant ensembles [1, 32, 33].

LEMMA 4.5. For any $n \geq 1$,

$$
\sum_{|\mathbf{m}|=n} \mathrm{M}(\mathbf{m})=\left\{\begin{array}{ll}
1 & \text { if } n=1, \\
0 & \text { if } n \geq 2,
\end{array} \text { and } \quad \sum_{|\mathbf{m}|=n}|\mathrm{M}(\mathbf{m})| \leq n ! 2^{n-1}\right.
$$

We have assumed that our test function $f$ has compact support since the original problem is to study mesoscopic linear statistics. However, this assumption is not necessary to prove Theorem 4.4. We shall certainly require that $f \in L^{1}(\mathbb{R})$ and, according to the estimate (4.16), the regularity condition needed to prove Theorem 4.4 is that, for any $n \geq 2$, the integral

$$
\int_{\mathbb{R}_{0}^{n}}\left|\hat{f}\left(u_{1}\right) \cdots \hat{f}\left(u_{n}\right)\right|\left(1+|u|_{1}\right) d^{n-1} u<\infty .
$$

A sufficient condition is provided by the next lemma since by assumption: $\|\hat{f}\|_{\infty} \leq$ $\|f\|_{L^{1}}<\infty$.

LEMMA 4.6. For any functions $f_{1}, \ldots, f_{n} \in H^{1}(\mathbb{R})$,

$$
\begin{aligned}
& \int_{\mathbb{R}_{0}^{n}}\left|\hat{f}_{1}\left(u_{1}\right) \cdots \hat{f}_{n}\left(u_{n}\right)\right|\left(1+\left|u_{1}\right|+\cdots+\left|u_{n}\right|\right) d^{n-1} u \\
& \quad \leq n 2^{n} \prod_{j=1}^{n}\left(\left\|\hat{f}_{j}\right\|_{\infty}+\left\|f_{j}\right\|_{H^{1}}\right)
\end{aligned}
$$

PROOF. By the Cauchy-Schwarz inequality,

$$
\begin{aligned}
& \int_{\mathbb{R}}\left|\hat{f}_{1}\left(u_{1}\right) \hat{f}_{2}\left(-\sum_{j \neq 2} u_{j}\right)\right|\left(1+\left|u_{1}\right|\right) d u_{1} \\
& \quad \leq 2\left(2\left\|\hat{f}_{1}\right\|_{\infty}\left\|\hat{f}_{2}\right\|_{\infty}+\int_{|u|>1}\left|\hat{f}_{1}(u) \hat{f}_{2}\left(-u-\sum_{j>2} u_{j}\right)\right||u| d u\right) \\
& \quad \leq 2\left(2\left\|\hat{f}_{1}\right\|_{\infty}\left\|\hat{f}_{2}\right\|_{\infty}+\left\|f_{1}\right\|_{H^{1}}\left\|f_{2}\right\|_{L^{2}}\right) .
\end{aligned}
$$

A similar argument shows that for any $f \in L^{1} \cap H^{1}(\mathbb{R})$,

$$
\|\hat{f}\|_{L^{1}} \vee\|\hat{f}\|_{L^{2}} \leq 2\left(\|\hat{f}\|_{\infty}+\|f\|_{H^{1}}\right)
$$


Hence, it follows from (4.24) that

$$
\int_{\mathbb{R}}\left|\hat{f}_{1}\left(u_{1}\right) \hat{f}_{2}\left(-\sum_{j \neq 2} u_{j}\right)\right|\left(1+\left|u_{1}\right|\right) d u_{1} \leq 4\left(\left\|\hat{f}_{1}\right\|_{\infty}+\left\|f_{1}\right\|_{H^{1}}\right)\left(\left\|\hat{f}_{2}\right\|_{\infty}+\left\|f_{2}\right\|_{H^{1}}\right),
$$

and, if we combine this estimate with (4.25),

$$
\begin{aligned}
& \int_{\mathbb{R}_{0}^{n}}\left|\hat{f}_{1}\left(u_{1}\right) \cdots \hat{f}_{n}\left(u_{n}\right)\right|\left(1+\left|u_{1}\right|\right) d^{n-1} u \\
& \quad \leq 4\left(\left\|\hat{f}_{1}\right\|_{\infty}+\left\|f_{1}\right\|_{H^{1}}\right)\left(\left\|\hat{f}_{2}\right\|_{\infty}+\left\|f_{2}\right\|_{H^{1}}\right) \prod_{j>2}\left\|\hat{f}_{j}\right\|_{L^{1}} \\
& \quad \leq 2^{n} \prod_{j=1}^{n}\left(\left\|\hat{f}_{j}\right\|_{\infty}+\left\|f_{j}\right\|_{H^{1}}\right) .
\end{aligned}
$$

The upper-bound (4.23) follows by symmetry.

The next lemma explains how the shape-dependent constant $B_{\Psi}^{n}$ defined by (1.29) arises in (4.20).

LEMMA 4.7. For any $n \geq 1$,

$$
\begin{aligned}
\mathrm{B}_{\Psi}^{n} & =\int_{\mathbb{R}_{<}^{n}} \prod_{i=1}^{n} \Phi\left(x_{i}\right) \sum_{\sigma \in \mathbb{S}(n)} \sum_{|\mathbf{m}|=n} \mathbf{M}(\mathbf{m}) x_{\sigma(\mathfrak{s})} d^{n} x \\
& =\sum_{k=0}^{n-1} b_{k}^{n} \int_{\mathbb{R}} z \Phi(z) \Psi(z)^{k}(1-\Psi(z))^{n-1-k} d z,
\end{aligned}
$$

where, according to formula (1.27), $x_{\sigma(\mathfrak{s})}=\min _{i \leq \ell(\mathbf{m})}\left\{x_{\sigma(i)}\right\}$ for all $x \in \mathbb{R}_{<}^{n}$, and the coefficients $b_{k}^{n}$ are given by formula (1.30).

PROOF. Let $\mathbb{P}_{n}$ be the uniform probability measure on the symmetric group $\mathbb{S}(n)$, so that we can view $\sigma\left(\mathfrak{s}_{l}\right)=\min _{i \leq l}\{\sigma(i)\}$ as a random variable. Then we can rewrite equation (4.26) as

$$
\mathrm{B}_{\Psi}^{n}=n ! \int_{\mathbb{R}_{<}^{n}} \prod_{i=1}^{n} \Phi\left(x_{i}\right) \sum_{|\mathbf{m}|=n} \mathbf{M}(\mathbf{m}) \mathbb{E}_{n}\left[x_{\sigma\left(\mathfrak{s}_{\ell}\right)}\right] d^{n} x .
$$

We claim that for any $l=1, \ldots, n$ and for any $k=0, \ldots, n-1$,

$$
\mathbb{P}\left[\min _{i \leq l} \sigma(i) \geq n-k\right]=\left(\begin{array}{c}
k+1 \\
l
\end{array}\right) \frac{l !(n-l) !}{n !} .
$$

To see this, observe that if $k+1<l$, since there are only $k$ elements in $\{1, \ldots, n\}$ which are greater than $n-k$, one of the $l$ first elements of $\sigma$ has to be less than $n-k$ and, therefore, the probability in question is 0 . 
On the other hand, if $l \leq k+1$, then $n-k$ is smaller than the minimum of the $l$ first entries of $\sigma$ if and only if these entries are drawn from the set $\{n-k, \ldots, n\}$. Since the order of these entries and that of the $(n-l)$ last entries is irrelevant, the number of such permutations is $\left(\begin{array}{c}k+1 \\ l\end{array}\right) l !(n-l)$ !. Hence, by definition and equation (4.28), the distribution of $\sigma\left(\mathfrak{s}_{l}\right)$ is

$$
\mathbb{P}_{n}\left[\sigma\left(\mathfrak{s}_{l}\right)=n-k\right]=\left(\begin{array}{l}
n \\
l
\end{array}\right)^{-1}\left(\begin{array}{c}
k \\
l-1
\end{array}\right) .
$$

Then, by definition of $\mathrm{M},(1.18)$,

$$
\begin{aligned}
& \sum_{|\mathbf{m}|=n} \operatorname{M}(\mathbf{m}) \mathbb{E}_{n}\left[x_{\sigma\left(\mathfrak{s}_{\ell}\right)}\right] \\
& \quad=\sum_{l=1}^{n} \frac{(-1)^{l+1}}{l}\left(\begin{array}{c}
n \\
l
\end{array}\right)^{-1} \sum_{\substack{|\mathbf{m}|=n \\
\ell(\mathbf{m})=l}}\left(\begin{array}{c}
n \\
\mathbf{m}
\end{array}\right) \sum_{k=0}^{n-1}\left(\begin{array}{c}
k \\
l-1
\end{array}\right) x_{n-k} .
\end{aligned}
$$

If we integrate successively over $x_{1}, \ldots, x_{n-k-1}$ and over $x_{n}, \ldots, x_{n-k+1}$, and use the relationship $\Phi=-\Psi^{\prime}$, we find that for any $k=0, \ldots, n-1$,

$$
\begin{aligned}
\int_{\mathbb{R}_{<}^{n}} & \prod_{i=1}^{n} \Phi\left(x_{i}\right) x_{n-k} d^{n} x \\
& =\frac{1}{k !(n-k-1) !} \int_{\mathbb{R}} \Psi(x)^{k}(1-\Psi(x))^{n-k-1} \Phi(x) x d x .
\end{aligned}
$$

Then, if we combine formulae (4.27), (4.29) and (4.30), we get

$$
\begin{aligned}
\mathrm{B}_{\Psi}^{n}= & \sum_{l=1}^{n}(-1)^{l+1} \frac{n !}{l !}\left(\begin{array}{c}
n \\
l
\end{array}\right)^{-1} \sum_{\substack{|\mathbf{m}|=n \\
\ell(\mathbf{m})=l}}\left(\begin{array}{c}
n \\
\mathbf{m}
\end{array}\right) \\
& \times \sum_{k=0}^{n-1} \frac{1}{(k-l+1) !(n-k-1) !} \int_{\mathbb{R}} \Psi(x)^{k}(1-\Psi(x))^{n-k-1} \Phi(x) x d x .
\end{aligned}
$$

We see that we can simplify $\frac{n !}{l !}\left(\begin{array}{c}n \\ l\end{array}\right)^{-1}$ from the previous formula and exchange the sums over $l$ and $k$. In the end, we obtain

$$
\begin{aligned}
\mathrm{B}_{\Psi}^{n}= & \sum_{k=0}^{n-1}\left(\sum_{l=1}^{n}(-1)^{l+1}\left(\begin{array}{c}
n-l \\
k+1-l
\end{array}\right) \sum_{\substack{|\mathbf{m}|=n \\
\ell(\mathbf{m})=l}}\left(\begin{array}{l}
n \\
\mathbf{m}
\end{array}\right)\right) \\
& \times \int_{\mathbb{R}} \Psi(x)^{k}(1-\Psi(x))^{n-k-1} \Phi(x) x d x .
\end{aligned}
$$

If we define the array $b_{k}^{n}$ according to (1.30), the lemma is proved. 
The cumulant problem is generally not discussed directly in the literature, so we provide a simple criterion which guarantees uniqueness of the law of a random variable given its cumulants.

LEMMA 4.8. Given a sequence of random variables $X_{N}$ whose Laplace transform is well defined and such that for any $n \geq 1, \mathrm{C}^{n}\left[X_{N}\right] \rightarrow \mathrm{C}_{\infty}^{n}$ as $N \rightarrow \infty$. If there exists constants $c, v>0$, such that

$$
\left|\mathrm{C}_{\infty}^{n}\right| \leq c n ! v^{n}
$$

then there exists a random variable $X_{\infty}$ whose cumulants satisfy $\mathrm{C}^{n}\left[X_{\infty}\right]=\mathrm{C}_{\infty}^{n}$ and the sequence $X_{N} \Rightarrow X$.

The condition of Lemma 4.8 is very natural and its proof follows from a straightforward repetition of the argument that is used when dealing with the Hamburger moment problem (see, e.g., Section 3.3.3 in [15]). Next, we use this criterion to deduce from Theorem 4.4 the weak convergence of linear statistics $\Xi f$ for any determinantal process with correlation kernel $L_{\Psi, \eta}^{N}$.

DEFINITION 4.9. In the sequel, the quantity $2 \tau \mathrm{B}_{\Psi}^{n} \int_{\mathbb{R}} f(t)^{n} d t$ will be called the Poisson component of the $n$th cumulant and we will use the decomposition $\lim _{N \rightarrow \infty} \mathrm{C}_{L_{\Psi, \eta}^{N}}^{n}[\Xi f]=2 \tau \mathrm{B}_{\Psi}^{n} \int_{\mathbb{R}} f(t)^{n} d t+\mathfrak{G}_{\Psi, \tau}^{n}[f]$ where

$$
\begin{aligned}
\mathfrak{G}_{\Psi, \tau}^{n}[f]= & -2 \int_{\mathbb{R}_{0}^{n}} d u \Re\left\{\prod_{i=1}^{n} \hat{f}\left(u_{i}\right)\right\} \\
& \times \int_{\mathbb{R}_{<}^{n}} \prod_{i=1}^{n} \Phi\left(x_{i}\right) \sum_{|\mathbf{m}|=n} \mathbf{M}(\mathbf{m}) \mathrm{G}_{\tau}^{\mathbf{m}}(u, x) d x .
\end{aligned}
$$

This name is motivated by linear statistics of the Poisson point process whose cumulants are equal to $\tau^{\prime} \int_{\mathbb{R}} f(t)^{n} d t$ where $\tau^{\prime}$ is the intensity.

COROLlaRY 4.10. Consider the determinantal process with correlation kernel $L_{\Psi, \eta}^{N}$ and let $f \in H_{0}^{1}(\mathbb{R})$. If the conditions (4.4)-(4.5) hold, then the random variable $\Xi f$ converges in distribution as $N \rightarrow \infty$ to a random variable $\Xi_{\Psi, \tau^{\prime}} f$ where $\tau^{\prime}=\beta \tau$ and whose cumulants are given by

$$
\mathrm{C}^{n}\left[\Xi_{\Psi, \tau^{\prime}} f\right]=2 \tau^{\prime} \mathrm{B}_{\Psi}^{n} \int_{\mathbb{R}} f(t)^{n} d t+\mathfrak{G}_{\Psi, \tau^{\prime}}^{n}[f]
$$

ProOF. We can estimate the growth of $\mathfrak{G}_{\Psi, \tau^{\prime}}^{n}[f]$ and the Poisson component separately. We start by giving an upper-bound for the constant $\mathrm{B}_{\Psi}^{n}$. By formula (4.27),

$$
\mathrm{B}_{\Psi}^{n}=n ! \int_{\mathbb{R}_{<}^{n}} \prod_{i=1}^{n} \Phi\left(x_{i}\right) \sum_{|\mathbf{m}|=n} \mathrm{M}(\mathbf{m}) \mathbb{E}_{n}\left[x_{\sigma\left(\mathfrak{s}_{\ell}\right)}\right] d^{n} x
$$


Obviously, for any $x \in \mathbb{R}_{<}^{n}, \mathbb{E}_{n}\left[x_{\sigma\left(\mathfrak{s}_{\ell}\right)}\right] \leq x_{n}$ and if we use formula (4.30),

$$
\begin{aligned}
\int_{\mathbb{R}_{<}^{n}} \prod_{i=1}^{n} \Phi\left(x_{i}\right) \mathbb{E}_{n}\left[x_{\mathfrak{s}_{\ell}}\right] d^{n} x & \leq \int_{\mathbb{R}_{<}^{n}} \prod_{i=1}^{n} \Phi\left(x_{i}\right) x_{n} d^{n} x \\
& =\frac{1}{(n-1) !} \int_{\mathbb{R}}(1-\Psi(x))^{n-1} \Phi(x) x d x .
\end{aligned}
$$

Moreover, since $0 \leq \Phi=-\Psi^{\prime}$ and $0 \leq \Psi \leq 1$, we have for any $n \geq 1$,

$$
\int_{\mathbb{R}}(1-\Psi(x))^{n-1} \Phi(x) x d x \leq \int_{0}^{\infty} \Phi(x) x d x=\int_{0}^{\infty} \Psi(x) d x .
$$

On the other hand, if we use that $x_{1} \leq \mathbb{E}_{n}\left[x_{\sigma\left(\mathfrak{s}_{\ell}\right)}\right]$ and apply the same method, we can show that

$$
\begin{aligned}
\int_{\mathbb{R}_{<}^{n}} \prod_{i=1}^{n} \Phi\left(x_{i}\right) \mathbb{E}_{n}\left[x_{\mathfrak{\mathfrak { F } _ { \ell }}}\right] d^{n} x & \geq \frac{1}{(n-1) !} \int_{\mathbb{R}} \Psi(x)^{n-1} \Phi(x) x d x \\
& \geq \frac{-\Psi(0)^{n-1}}{(n-1) !} \int_{-\infty}^{0}(1-\Psi(x)) d x .
\end{aligned}
$$

These estimates show that there exists a positive constant $C$ which only depends on the shape $\Psi$ such that for any $l=1, \ldots, n$,

$$
n !\left|\int_{\mathbb{R}_{<}^{n}} \prod_{i=1}^{n} \Phi\left(x_{i}\right) \mathbb{E}_{n}\left[x_{\sigma\left(\mathfrak{s}_{l}\right)}\right] d^{n} x\right| \leq C n .
$$

Using the estimate of Lemma 4.5 and (4.27), this implies that $\left|\mathrm{B}_{\Psi}^{n}\right| \leq C(n+$ 1)! $2^{n-1}$. Hence, for any $n \geq 2$, the Poisson component is bounded by

$$
\left|\mathrm{B}_{\Psi}^{n} \int_{\mathbb{R}} f(t)^{n} d t\right| \leq C^{\prime}(n+1) !\left(2\|f\|_{\infty}\right)^{n-1}\|f\|_{L^{1}} .
$$

Now, we estimate the growth of $\mathfrak{G}_{\Psi, \tau^{\prime}}^{n}[f]$, (4.31). Applying the upper-bound (4.21), we see that

$$
\begin{aligned}
\left|\mathfrak{G}_{\Psi, \tau^{\prime}}^{n}[f]\right| \leq & 2 n ! \sum_{|\mathbf{m}|=n}|\mathbf{M}(\mathbf{m})| \\
& \times \int_{R_{0}^{n}} \prod_{i=1}^{n}\left|\hat{f}\left(u_{i}\right)\right|\left(\left|u_{1}\right|+\cdots+\left|u_{n}\right|\right) d^{n-1} u \int_{\mathbb{R}_{<}^{n}} \prod_{i=1}^{n} \Phi\left(x_{i}\right) d^{n} x .
\end{aligned}
$$

By symmetry $\int_{\mathbb{R}_{<}^{n}} \prod_{i=1}^{n} \Phi\left(x_{i}\right) d^{n} x=\frac{1}{n !}\left(\int_{\mathbb{R}} \Phi(x) d x\right)^{n}=\frac{1}{n !}$ and Lemmas 4.5 and 4.6 provide bounds for the other factors of the RHS of (4.34). We obtain

$$
\left|\mathfrak{G}_{\Psi, \tau^{\prime}}^{n}[f]\right| \leq(n+1) ! 4^{n}\left(\|f\|_{\infty}+\|f\|_{H^{1}}\right)^{n} .
$$

The estimates (4.33) and (4.35) show that the limits of Theorem 4.4 satisfy the criterion of Lemma 4.8 for any choice of parameters $\tau^{\prime}>0, \Psi \in \mathfrak{F}$, and $f \in H_{0}^{1}(\mathbb{R})$. 
Hence, they corresponds to the cumulants of some random variable which we denote by $\Xi_{\Psi, \tau^{\prime}} f$, and $\Xi f \Rightarrow \Xi_{\Psi, \tau^{\prime}} f$ as $N \rightarrow \infty$.

For the critical modified CUEs, according to formulae (4.3)(i) and (4.4), the parameter $\beta=1$. Hence, Theorem 2.7 follows directly from Proposition 2.4 and Corollary 4.10. Likewise, in the GUE setting, the parameter $\beta=1 / 4$ by formula (4.3)(ii). Provided that $1 / 3<\alpha<1$, by Proposition 1.11 , we conclude that at the critical scale, a modified GUE with shape $\Psi \in \mathfrak{E}$ converges in distribution to the random field $\Xi_{\Psi, \tau / 4}$. In order to deal with all mesoscopic scales, we can use the asymptotic expansion of Theorem A.2 instead of Theorem A.3. Namely, if we combine formula (3.10) with the sine-kernel approximation (A.10), we obtain for any scales $0<\alpha, \delta<1$,

$$
\begin{aligned}
N^{-\delta} & K_{\Psi, \alpha}^{N}\left(x N^{-\delta}, y N^{-\delta}\right) \\
= & \frac{1}{\tau N^{\alpha}} \sum_{|k| \leq \Gamma_{N} N^{\alpha}} \Phi\left(\frac{k}{\tau N^{\alpha}}\right) \\
& \times \frac{\sin \left[(N+k)\left(F\left(\frac{\pi}{2} \frac{x N^{1 / 2-\delta}}{\sqrt{N+k}}\right)-F\left(\frac{\pi}{2} \frac{y N^{1 / 2-\delta}}{\sqrt{N+k}}\right)\right)\right]}{\pi(x-y)}+\underset{N \rightarrow \infty}{O}\left(N^{-\delta}\right) .
\end{aligned}
$$

The RHS of (4.36) does not corresponds to a translation-invariant, so we cannot defined its Fourier transform. However, it is related to the kernel $L_{\Psi, \eta}^{N},(1.33)$, by a change of variables and we can exploit this fact to compute the limits of critical linear statistics of the modified GUE at any scale, including the regime $0<\alpha \leq 1 / 3$.

Proposition 4.11. Let $\Psi \in \mathfrak{E}, f \in C_{0}(\mathbb{R})$, and $0<\alpha<1$. We have

$$
\lim _{N \rightarrow \infty} \mathrm{C}_{K_{\Psi, \alpha}^{N}}^{n}\left[\Xi f_{\alpha}\right]=\lim _{N \rightarrow \infty} \mathrm{C}_{L_{\Psi, \eta}^{N}}^{n}\left[\Xi g_{N}\right]
$$

for any $n \geq 2$ where

$$
g_{N}(x)=f\left(\frac{2}{\pi} N^{\alpha} G\left(\frac{\pi x}{N^{\alpha}}\right)\right)
$$

and the function $G$ is given by Definition A.1.

ProOF. Let $\operatorname{supp}(f) \subset[-L, L]$. Observe that for any $|k| \leq \Gamma_{N} N^{\alpha}$ and any $x, y \in[-2 L, 2 L]$, a Taylor expansion gives

$$
\begin{aligned}
F\left(x \frac{N^{1 / 2-\alpha}}{\sqrt{N+k}}\right)-F\left(y \frac{N^{1 / 2-\alpha}}{\sqrt{N+k}}\right)= & \sqrt{\frac{N}{N+k}}\left\{F\left(\frac{x}{N^{\alpha}}\right)-F\left(\frac{y}{N^{\alpha}}\right)\right\} \\
& +\underset{N \rightarrow \infty}{O}\left((x-y) \frac{\Gamma_{N} N^{-\alpha}}{N+k}\right) .
\end{aligned}
$$


Thus, taking $\delta=\alpha$ in equation (4.36), we get for any $0<\alpha<1$,

$$
\begin{aligned}
N^{-\alpha} & K_{\Psi, \alpha}^{N}\left(x N^{-\alpha}, y N^{-\alpha}\right) \\
= & \frac{1}{\tau N^{\alpha}} \sum_{|k| \leq \Gamma N^{\alpha}} \Phi\left(\frac{k}{\tau N^{\alpha}}\right) \\
& \quad \times \frac{\sin \left[\sqrt{N(N+k)}\left(F\left(\frac{\pi}{2} \frac{x}{N^{\alpha}}\right)-F\left(\frac{\pi}{2} \frac{y}{N^{\alpha}}\right)\right)\right]}{\pi(x-y)}+\underset{N \rightarrow \infty}{O}\left(N^{-\alpha}\right),
\end{aligned}
$$

where the error term is uniform for all $x, y \in[-2 L, 2 L]$. Following the proof of Lemma 2.6, this approximation implies that for any any composition $\mathbf{m}$,

$$
\begin{aligned}
\operatorname{Tr}\left[f_{\alpha}^{m_{1}}\right. & \left.K_{\Psi, \alpha}^{N} \cdots f_{\alpha}^{m_{\ell}} K_{\Psi, \alpha}^{N}\right] \\
= & \sum_{\substack{k \in \mathbb{Z}^{\ell} \\
\left|k_{j}\right| \leq \Gamma_{N} N^{\alpha}}} \prod_{j=1}^{\ell} \Phi\left(\frac{k_{j}}{\tau N^{\alpha}}\right) \int_{[-L, L]^{\ell}} \prod_{j=1}^{\ell} f\left(x_{j}\right)^{m_{j}} \\
& \times \frac{\sin \left[\sqrt{N(N+k)}\left(F\left(\frac{\pi}{2} \frac{x_{j}}{N^{\alpha}}\right)-F\left(\frac{\pi}{2} \frac{x_{j+1}}{N^{\alpha}}\right)\right)\right]}{\pi\left(x_{j}-x_{j+1}\right)} d^{\ell} x+\underset{N \rightarrow \infty}{\bar{O}}\left(N^{-\alpha}\right),
\end{aligned}
$$

where $x_{\ell+1}=x_{1}$. There exists $N_{L} \in \mathbb{N}$ such that for all $N \geq N_{L}$, we can make the change of variables $y_{j}=\pi^{-1} N^{\delta} F\left(\frac{\pi}{2} \frac{x_{j}}{N^{\delta}}\right)$ in the integral (4.39). Since $0<F^{\prime}(x) \leq$ 2 for any $|x|<1$, this change of variables maps the interval $\left|x_{j}\right|<L$ to some subset of $\left|y_{j}\right|<L$ for any $N \geq N_{L}$. Hence, if we let $g_{N}(y)=f\left(\frac{2}{\pi} N^{\alpha} G\left(\frac{\pi y}{N^{\alpha}}\right)\right)$ and $\eta(k)$ is given by (4.3)(ii), we obtain

$$
\begin{array}{r}
\int_{[-L, L]^{\ell}} \prod_{j=1}^{\ell} f\left(x_{j}\right)^{m_{j}} \frac{\sin \left[\sqrt{N(N+k)}\left(F\left(\frac{\pi}{2} \frac{x_{j}}{N^{\alpha}}\right)-F\left(\frac{\pi}{2} \frac{x_{j+1}}{N^{\alpha}}\right)\right)\right]}{\pi\left(x_{j}-x_{j+1}\right)} d^{\ell} x \\
=\int_{[-L, L]^{\ell}} \prod_{j=1}^{\ell} g_{N}\left(y_{j}\right)^{m_{j}} \frac{G^{\prime}\left(\frac{\pi y_{j}}{N^{\alpha}}\right) \sin \left[2 \pi \eta(k)\left(y_{j}-y_{j+1}\right)\right]}{N^{\alpha}\left(G\left(\frac{\pi y_{j}}{N^{\alpha}}\right)-G\left(\frac{\pi y_{j+1}}{N^{\alpha}}\right)\right)} d^{\ell} x .
\end{array}
$$

A Taylor expansion gives for any $y, z \in[-4 L, 4 L]$,

$$
\frac{G\left(y N^{-\alpha}\right)-G\left(z N^{-\alpha}\right)}{N^{-\alpha} G^{\prime}\left(y N^{-\alpha}\right)}=(y-z)\left\{1+\underset{N \rightarrow \infty}{O}\left((y-z) N^{-\alpha}\right)\right\} .
$$

This implies that for any $|k| \leq \Gamma N^{\alpha}$,

$$
\frac{G^{\prime}\left(\frac{\pi y}{N^{\alpha}}\right) \sin [2 \pi \eta(k)(y-z)]}{N^{\alpha}\left(G\left(\frac{\pi y}{N^{\alpha}}\right)-G\left(\frac{\pi z}{N^{\alpha}}\right)\right)}=\frac{\sin [2 \pi \eta(k)(y-z)]}{\pi(y-z)}+\underset{N \rightarrow \infty}{O}\left(N^{-\alpha}\right) .
$$


Hence, if we combine formulae (4.39), (4.41) and (4.41), we have proved that

$$
\begin{aligned}
\operatorname{Tr}\left[f_{\alpha}^{m_{1}} K_{\Psi, \alpha}^{N} \cdots f_{\alpha}^{m_{\ell}} K_{\Psi, \alpha}^{N}\right] & \\
= & \sum_{\substack{k \in \mathbb{Z}^{\ell} \\
\left|k_{j}\right| \leq \Gamma N^{\alpha}}} \prod_{j=1}^{\ell} \Phi\left(\frac{k_{j}}{\tau N^{\alpha}}\right) \int_{[-L, L]^{\ell}} \prod_{j=1}^{\ell} g_{N}\left(y_{j}\right)^{m_{j}} \frac{\sin [2 \pi \eta(k)(y-z)]}{\pi(y-z)} d^{\ell} y \\
& +\underset{N \rightarrow \infty}{\bar{O}}\left(N^{-\alpha}\right) .
\end{aligned}
$$

By (1.33), we can write this equation as

$$
\operatorname{Tr}\left[f_{\alpha}^{m_{1}} K_{\Psi, \alpha}^{N} \cdots f_{\alpha}^{m_{\ell}} K_{\Psi, \alpha}^{N}\right]=\operatorname{Tr}\left[g_{N}^{m_{1}} L_{\Psi, \eta}^{N} \cdots g_{N}^{m_{\ell}} L_{\Psi, \eta}^{N}\right]+\underset{N \rightarrow \infty}{\bar{O}}\left(N^{-\alpha}\right),
$$

and the proposition follows from formula (1.19).

By Proposition 4.11, to complete the proof of Theorem 1.7, it remains to extend the argument of Theorem 4.4 to deal with test functions of the form (4.37). As we can see from the next lemma, such functions depend mildly on the density $N$ and it is not difficult to complete the proof.

LEMMA 4.12. Let $f \in C^{1}(\mathbb{R})$ with support in $[-L, L]$ and $0<\delta \leq 1$. For any $N>(2 L)^{1 / \delta}$, the function $g_{N}(x)=f\left(\frac{2}{\pi} N^{\delta} G\left(\frac{\pi x}{N^{\delta}}\right)\right)$ has compact support in $[-L, L]$. Moreover, we have

$$
\left\|\hat{g}_{N}-\hat{f}\right\|_{\infty}=\underset{N \rightarrow \infty}{O}\left(N^{-\delta}\right) \quad \text { and } \quad\left\|g_{N}-f\right\|_{H^{1}}=\underset{N \rightarrow \infty}{o(1)} .
$$

Proof. By Definition A.1, $0 \leq F^{\prime} \leq 2$ and the map $x \mapsto \frac{2}{\pi} N^{\delta} G\left(\frac{\pi x}{N^{\delta}}\right)$ is a dilation. Therefore, when $N>(2 L)^{1 / \delta}$, the function $g_{N}$ is well defined on $[-L, L]$ and $g_{N}(x)=0$ for all $x \in[-L, L] \backslash \operatorname{supp}(f)$. By continuity, we can assume that $g_{N}(x)=0$ for all $|x|>L$. Hence, $g_{N} \in C_{0}^{1}(\mathbb{R})$ with $\operatorname{supp}\left(g_{N}\right) \subset \operatorname{supp}(f)$. Then, by Lipschitz continuity of $f$, for any $|x|<L$,

$$
\left|g_{N}(x)-f(x)\right| \leq C N^{\delta}\left|2 G\left(\frac{\pi x}{N^{\delta}}\right)-\frac{\pi x}{N^{\delta}}\right| \leq C L^{2} N^{-\delta},
$$

where we used that $G$ is smooth with $G(0)=0$ and $G^{\prime}(0)=1 / 2$. This implies that

$$
\left\|\hat{g}_{N}-\hat{f}\right\|_{\infty} \leq \int_{[-L, L]}\left|g_{N}(x)-f(x)\right| d x=O\left(N^{-\delta}\right) .
$$

Similarly, by the triangle inequality,

$$
\left|g_{N}^{\prime}(x)-f^{\prime}(x)\right| \leq\left\|f^{\prime}\right\|_{\infty}\left|2 G^{\prime}\left(\frac{\pi x}{N^{\delta}}\right)-1\right|+\left|f^{\prime}\left(\frac{2}{\pi} N^{\delta} G\left(\frac{\pi x}{N^{\delta}}\right)\right)-f^{\prime}(x)\right| .
$$


Since $f^{\prime}$ is continuous, $\lim _{N \rightarrow \infty}\left|g_{N}^{\prime}(x)-f^{\prime}(x)\right|=0$ for all $x \in[-L, L]$. By (1.21) and the dominated convergence theorem, we conclude that as $N \rightarrow \infty$,

$$
\left\|g_{N}-f\right\|_{H^{1}}^{2}=\frac{1}{4 \pi^{2}} \int_{[-L, L]}\left|g_{N}^{\prime}(x)-f^{\prime}(x)\right|^{2} d x \rightarrow 0 .
$$

Proposition 4.13. Let $f \in C_{0}^{1}(\mathbb{R}), 0<\alpha<1$, and $\Psi \in \mathfrak{E}$. If $\eta$ is given by (4.3)(ii) and $g_{N}$ is given by (4.37), then for any $n \geq 2$,

$$
\lim _{N \rightarrow \infty} \mathrm{C}_{L_{\Psi, \eta}^{N}}^{n}\left[\Xi g_{N}\right]=\mathrm{C}^{n}\left[\Xi_{\Psi, \tau / 4} f\right] .
$$

PROOF. We can take $f=g_{N}$ in formula (4.8). In particular, the estimate (4.16) is still valid for the test function $g_{N}$. Let $|u|_{1}=\left|u_{1}\right|+\cdots+\left|u_{n}\right|$ and the function $\Upsilon_{N}^{n}(u)$ be given by (4.12). We need to prove that

$$
\lim _{N \rightarrow \infty} \int_{\mathbb{R}_{0}^{n}} \mathbb{1}_{\left\{|u|_{1}>\frac{N^{1}-\alpha}{2}\right\}}|u|_{1} \prod_{i}\left|\hat{g}_{N}\left(u_{i}\right)\right| d^{n-1} u=0,
$$

and

$$
\lim _{N \rightarrow \infty} \int_{\mathbb{R}_{0}^{n}}\left|\prod_{i} \hat{g}_{N}\left(u_{i}\right)-\prod_{i} \hat{f}\left(u_{i}\right)\right|\left|\Upsilon_{N}^{n}(u)\right| d^{n-1} u=0 .
$$

Indeed these limits imply that

$$
\begin{aligned}
\lim _{N \rightarrow \infty} C_{L_{\Psi, \eta}^{N}}^{n}\left[\Xi g_{N}\right] & =2 \lim _{N \rightarrow \infty} \int_{\mathbb{R}_{0}^{n}} \Re\left\{\prod_{i} \hat{g}_{N}\left(u_{i}\right)\right\} \Upsilon_{N}^{n}(u) d^{n-1} u \\
& =2 \lim _{N \rightarrow \infty} \int_{\mathbb{R}_{0}^{n}} \Re\left\{\prod_{i} \hat{f}\left(u_{i}\right)\right\} \Upsilon_{N}^{n}(u) d^{n-1} u,
\end{aligned}
$$

and the rest of the proof is identical to that of Theorem 4.4. To complete our argument, it remains to show (4.42) and (4.43). First, observe that

$$
\begin{aligned}
\int_{\mathbb{R}_{0}^{n}} \mathbb{1}_{\left\{|u|_{1}>\frac{N^{1}-\alpha}{2}\right\}}|u|_{1} \prod_{i}\left|\hat{g}_{N}\left(u_{i}\right)\right| d^{n-1} u \\
\quad \leq \sum_{k=1}^{n} \int_{\mathbb{R}_{0}^{n}} \mathbb{1}_{\left\{\left|u_{k}\right|>\frac{N^{1-\alpha}}{2 n}\right\}}|u|_{1} \prod_{i}\left|\hat{g}_{N}\left(u_{i}\right)\right| d^{n-1} u .
\end{aligned}
$$

Let $\mathcal{A}_{N}=\left\{v \in \mathbb{R}:|v|>\frac{N^{1-\alpha}}{2 n}\right\}$ and define the function $q_{N}$ by its Fourier transform $\hat{q}_{N}=\mathbb{1}_{\mathcal{A}_{N}} \hat{g}_{N}$. Then by Lemma 4.6 we have

$$
\begin{aligned}
& \int_{\mathbb{R}_{0}^{n}} \mathbb{1}_{\left\{|u|_{1}>\frac{N^{1}-\alpha}{2}\right\}}|u|_{1} \prod_{i}\left|\hat{g}_{N}\left(u_{i}\right)\right| d^{n-1} u \\
& \leq n^{2} 2^{n-1}\left(\left\|\hat{q}_{N}\right\|_{\infty}+\left\|q_{N}\right\|_{H^{1}}\right)\left(\left\|\hat{g}_{N}\right\|_{\infty}+\left\|g_{N}\right\|_{H^{1}}\right)^{n-1} .
\end{aligned}
$$


Since, $\left\|F^{\prime}\right\|_{\infty} \leq 2$, a change of variables yields

$$
\left\|\hat{g}_{N}\right\|_{\infty} \leq \int\left|f\left(\frac{2}{\pi} N^{\alpha} G\left(\frac{\pi x}{N^{\alpha}}\right)\right)\right| d x \leq \int|f(z)| d z=\|f\|_{L^{1}} .
$$

Moreover,

$$
\left\|g_{N}\right\|_{H^{1}}^{2}=\int\left|g_{N}^{\prime}(x)\right|^{2} d x=2 \int_{-L}^{L}\left|f^{\prime}(z)\right|^{2} G^{\prime}\left(F\left(\frac{\pi z}{2 N^{\alpha}}\right)\right) d z,
$$

and, since $G^{\prime}(F(t))=F^{\prime}(t)=2 \sqrt{1-t^{2}}$ for any $|t|<1$, we obtain that $\left\|g_{N}\right\|_{H^{1}}^{2} \leq$ $4\|f\|_{H^{1}}^{2}$ for all $N$ sufficiently large. By (4.45), this implies that

$$
\begin{aligned}
\int_{\mathbb{R}_{0}^{n}} \mathbb{1}_{\left\{|u|_{1}>\frac{N^{1-\alpha}}{2}\right\}}|u| \prod_{i}\left|\hat{g}_{N}\left(u_{i}\right)\right| d^{n-1} u \\
\quad \leq n^{2} 2^{n-1}\left(\left\|\hat{q}_{N}\right\|_{\infty}+\left\|q_{N}\right\|_{H^{1}}\right)\left(\|\hat{f}\|_{L^{1}}+2\|f\|_{H^{1}}\right)^{n-1} .
\end{aligned}
$$

Obviously, $\left\|\hat{q}_{N}\right\|_{\infty} \rightarrow 0$ and to conclude that (4.42) holds it remains to estimate $\left\|q_{N}\right\|_{H^{1}}$. The main observation is that

$$
\int\left|\hat{q}_{N}(v)-\mathbb{1}_{\mathcal{A}_{N}} \hat{f}(v)\right|^{2}|v|^{2} d v \leq\left\|g_{N}-f\right\|_{H^{1}}^{2} .
$$

Then by the triangle inequality

$$
\left\|q_{N}\right\|_{H^{1}}^{2} \leq 2\left(\left\|g_{N}-f\right\|_{H^{1}}^{2}+\int_{\mathcal{A}_{N}}|\hat{f}(v)|^{2}|v|^{2} d v\right) .
$$

The first term converges to 0 by Lemma 4.12, and so does the second term by the dominated convergence theorem. We conclude that $\left\|q_{N}\right\|_{H^{1}} \rightarrow 0$ and we have established (4.42). The proof of the estimate (4.43) is very similar. We observe that

$$
\prod_{i} \hat{g}_{N}\left(u_{i}\right)-\prod_{i} \hat{f}\left(u_{i}\right)=\sum_{j=1}^{n}\left(\hat{g}_{N}\left(u_{j}\right)-\hat{f}\left(u_{j}\right)\right) \prod_{i>j} \hat{g}_{N}\left(u_{i}\right) \prod_{i<j} \hat{f}\left(u_{i}\right) .
$$

By (4.22), there exists a constant $C>0$ which depends only on $n$ and the shape $\Psi$ such that $\left|\Upsilon_{N}^{n}(u)\right| \leq C\left\{1+|u|_{1}\right\}$ and we obtain

$$
\begin{aligned}
\int_{\mathbb{R}_{0}^{n}} \mid & \prod_{i} \hat{g}_{N}\left(u_{i}\right)-\prod_{i} \hat{f}\left(u_{i}\right)|| \Upsilon_{N}^{n}(u) \mid d^{n-1} u \\
& \leq C \sum_{j=1}^{n} \int_{\mathbb{R}_{0}^{n}}\left|\hat{g}_{N}\left(u_{j}\right)-\hat{f}\left(u_{j}\right)\right|\left|\prod_{i>j} \hat{g}_{N}\left(u_{i}\right) \prod_{i<j} \hat{f}\left(u_{i}\right)\right|\left\{1+|u|_{1}\right\} d^{n-1} u .
\end{aligned}
$$

Thus, by Lemma 4.6 and the facts $\left\|\hat{g}_{N}\right\|_{\infty} \leq\|f\|_{L^{1}}$ and $\left\|g_{N}\right\|_{H^{1}} \leq 2\|f\|_{H^{1}}$, we get

$$
\begin{aligned}
\int_{\mathbb{R}_{0}^{n}} \mid & \prod_{i} \hat{g}_{N}\left(u_{i}\right)-\prod_{i} \hat{f}\left(u_{i}\right)|| \Upsilon_{N}^{n}(u) \mid d^{n-1} u \\
& \leq C n^{2} 2^{n-1}\left(\left\|\hat{g}_{N}-\hat{f}\right\|_{\infty}+\left\|g_{N}-f\right\|_{H^{1}}\right)\left(\|f\|_{L^{1}}+2\|f\|_{H^{1}}\right)^{n-1} .
\end{aligned}
$$


Lemma 4.12 implies that the RHS of (4.47) converges to 0 as $N \rightarrow \infty$ and the limit (4.43) follows.

By Propositions 4.11 and 4.13, if $f \in C_{0}^{1}(\mathbb{R})$, we have for any $0<\alpha<1$ and any $n \geq 2$,

$$
\lim _{N \rightarrow \infty} \mathrm{C}_{K_{\Psi, \alpha}^{N}}^{N}\left[\Xi f_{\alpha}\right]=\mathrm{C}^{n}\left[\Xi_{\Psi, \tau / 4} f\right] .
$$

In the proof of Corollary 4.10, we have established that the sequence $\left(C^{n}\left[\Xi_{\Psi, \tau / 4} f\right]\right)_{n \geq 2}$ satisfies the condition of Lemma 4.8. This implies that, considering the determinantal process with correlation kernel $K_{\Psi, \alpha}^{N}$, the random variable

$$
\Xi f_{\alpha} \Rightarrow \Xi_{\Psi, \tau / 4} f
$$

as $N \rightarrow \infty$. In order to complete the proof of Theorem 1.7, we use a density argument to extend (4.49) to all test functions in $H_{0}^{1 / 2} \cap L^{\infty}(\mathbb{R})$.

Proof of TheOREM 1.7. Let $\tau^{\prime}=\tau / 4$. First, observe that for any $x, y \in \mathbb{R}$,

$$
\left|e^{i x}-e^{i y}\right|^{2} \leq 4|x-y|^{2} \text {. }
$$

By Chebyshev's inequality, this implies that, if $X$ and $Y$ are mean-zero random variables defined on the same probability space, for any $\xi \in \mathbb{R}$,

$$
\left|\mathbb{E}\left[e^{i \xi X}-e^{i \xi Y}\right]\right| \leq 4|\xi| \sqrt{\operatorname{Var}[X-Y]} .
$$

For critical linear statistics of the modified GUEs, using the estimate (3.26) which is valid when $\delta=\alpha$, we obtain for any test functions $f, h \in H_{0}^{1 / 2}(\mathbb{R})$,

$$
\left|\mathbb{E}_{K_{\Psi, \alpha}^{N}}\left[e^{i \xi \Xi f_{\alpha}}-e^{i \xi \Xi h_{\alpha}}\right]\right| \leq C|\xi| \sqrt{\|f-h\|_{L^{2}}^{2}+\|f-h\|_{H^{1 / 2}}^{2}} .
$$

Moreover, formula (B.4) implies that under the same assumptions,

$$
\left|\mathbb{E}\left[e^{i \xi \Xi_{\Psi, \tau^{\prime}} h}-e^{i \xi \Xi_{\Psi, \tau^{\prime}} f}\right]\right| \leq C|\xi| \sqrt{\|f-h\|_{L^{2}}^{2}+\|f-h\|_{H^{1 / 2}}^{2}} .
$$

By the triangle inequality,

$$
\begin{aligned}
& \left|\mathbb{E}_{K_{\Psi, \alpha}^{N}}\left[e^{i \xi \Xi f_{\alpha}}\right]-\mathbb{E}\left[e^{i \xi \Xi_{\Psi, \tau^{\prime}} f}\right]\right| \\
& \leq\left|\mathbb{E}_{K_{\Psi, \alpha}^{N}}\left[e^{i \xi \Xi f_{\alpha}}-e^{i \xi \Xi h_{\alpha}}\right]\right|+\left|\mathbb{E}\left[e^{i \xi \Xi_{\Psi, \tau^{\prime}} h}-e^{i \xi \Xi_{\Psi, \tau^{\prime}} f}\right]\right| \\
& \quad+\left|\mathbb{E}_{K_{\Psi, \alpha}^{N}}\left[e^{i \xi \Xi h_{\alpha}}\right]-\mathbb{E}\left[e^{i \xi \Xi_{\Psi, \tau^{\prime}} h}\right]\right| .
\end{aligned}
$$

If we suppose that $h \in C_{0}^{1}(\mathbb{R})$, by (4.49), the last term in the RHS of (4.52) converges to 0 as $N \rightarrow \infty$. Thus, using the upper-bound (4.50) and (4.51), for any $f \in H_{0}^{1 / 2} \cap L^{\infty}(\mathbb{R})$ and $\xi \in \mathbb{R}$,

$$
\begin{aligned}
& \limsup _{N \rightarrow \infty}\left|\mathbb{E}_{K_{\Psi, \alpha}^{N}}\left[e^{i \xi \Xi f_{\alpha}}\right]-\mathbb{E}\left[e^{i \xi \Xi_{\Psi, \tau^{\prime}} f}\right]\right| \\
& \leq 2 C|\xi| \sqrt{\|f-h\|_{L^{2}}^{2}+\|f-h\|_{H^{1 / 2}}^{2}} .
\end{aligned}
$$


Since, the space $C_{0}^{1}$ is dense in the Sobolev space $H_{0}^{1 / 2}$ with respect to the norm $\sqrt{\|\cdot\|_{L^{2}}^{2}+\|\cdot\|_{H^{1 / 2}}^{2}},[28]$, Theorem 7.14, the RHS of the inequality (4.53) is arbitrary small by choosing $h \in C_{0}^{1}(\mathbb{R})$ appropriately, and we conclude that $\Xi f_{\alpha} \Rightarrow \Xi_{\Psi, \tau^{\prime}} f$ as $N \rightarrow \infty$.

4.3. Properties of the random process $\Xi_{\Psi, \tau}$. In this section, we study the random variables $\Xi_{\Psi, \tau} f$ which arise from the limit of linear statistics of the critical modified ensembles. Because of the complicated structure of the cumulants in Theorem 1.7, we cannot get much information about the random fields $\Xi_{\Psi, \tau}$ except that they are not Gaussian. However, as we expect from Figure 1, we recover Gaussian fluctuations in both limits $\tau \rightarrow \infty$ and $\tau \rightarrow 0$; see Proposition 4.15. Proposition 4.14 provides a sufficient condition under which the field $\Xi_{\Psi, \tau}$ is not Gaussian and it leads us to compute the Laplace transform of the Poisson component of the field $\Xi_{\Psi, \tau}$. Moreover, we show that the Poisson component of the random field $\Xi_{\Psi, \tau}$ is Gaussian if and only if $\Psi$ is the MNS shape $\psi(t)=\left(1+e^{t}\right)^{-1}$; see Proposition 1.9. Then a natural problem that remains unanswered is whether the sequence $\mathfrak{G}_{\psi, \tau}$ given by (4.31) also corresponds to the cumulants of some random variables, so that the field $\Xi_{\psi, \tau}$ would be the superposition of a white noise and an independent non-Gaussian process.

PROPOSITION 4.14. If the shape $\Psi \in \mathfrak{F}$ satisfies the condition $\mathrm{B}_{\Psi}^{n} \neq 0$ for some $n>2$. Then, for any $\tau>0$, the random process $\Xi_{\Psi, \tau}$ defined in Corollary 4.10 is not Gaussian.

PROOF. It is clear from the definition (1.28) that $\lim _{\tau \rightarrow \infty} \mathrm{G}_{\tau}^{\mathbf{m}}(u, x)=0$ and it follows from (4.31) that for any $n \geq 2$ and $f \in H_{0}^{1}(\mathbb{R}), \lim _{\tau \rightarrow \infty} \mathfrak{G}_{\Psi, \tau}^{n}(f)=0$. Hence, by formula (4.32),

$$
\mathrm{C}^{n}\left[\Xi_{\Psi, \tau} f\right]=2 \tau \mathrm{B}_{\Psi}^{n} \int_{\mathbb{R}} f(t)^{n} d t+\underset{\tau \rightarrow \infty}{o(1)} .
$$

Thus, the Poisson component dominates at large temperature and the random field of $\Xi_{\Psi, \tau} f$ is not Gaussian since there are test functions such that $\mathrm{C}^{n}\left[\Xi_{\Psi, \tau} f\right] \neq$ 0 whenever $\mathrm{B}_{\Psi}^{n} \neq 0$. This observation is actually valid at any temperature $\tau>$ 0 because of the scaling property of the cumulants. By definition, $\mathrm{G}_{\tau}^{\mathbf{m}}(u, x)=$ $\tau \mathrm{G}_{1}^{\mathbf{m}}(u / \tau, x)$ and the change of variables $u_{i}=\tau v_{i}$ leads to

$$
\mathfrak{G}_{\Psi, \tau}^{n}[f]=-2 \int_{\mathbb{R}_{0}^{n}} d v \Re\left\{\prod_{i=1}^{n} \tau \hat{f}\left(\tau v_{i}\right)\right\} \int_{\mathbb{R}_{<}^{n}} \prod_{i=1}^{n} \Phi\left(x_{i}\right) \sum_{|\mathbf{m}|=n} \mathbf{M}(\mathbf{m}) \mathrm{G}_{1}^{\mathbf{m}}(v, x) d x .
$$

Hence, by (4.32), the random variables $\Xi_{\Psi, \tau} f$ and $\Xi_{\Psi, 1} f\left(\frac{\dot{\tau}}{)}\right)$ have the same distribution. 
PROPOSITION 4.15. For any function $f \in H_{0}^{1}(\mathbb{R})$, the rescaled random variable $\tau^{-1 / 2} \Xi_{\Psi, \tau} f$ converges in distribution as $\tau \rightarrow \infty$ to a Gaussian random variable with variance $\|f\|_{L^{2}}^{2}$. On the other hand, $\Xi_{\Psi, \tau} f$ converges in distribution as $\tau \rightarrow 0$ to a Gaussian random variable with variance $\|f\|_{H^{1 / 2}}^{2}$.

ProOF. When $\tau \rightarrow \infty$, the asymptotic of the cumulants of the random variables $\Xi_{\Psi, \tau} f$ are given by formula (4.54) and

$$
\mathrm{C}^{n}\left[\Xi_{\Psi, \tau}\left(\tau^{-1 / 2} f\right)\right]=\tau^{1-n / 2} \mathrm{~B}_{\Psi}^{n} \int_{\mathbb{R}} f(t)^{n} d t+\underset{\tau \rightarrow \infty}{o}\left(\tau^{-n / 2}\right) .
$$

Hence, we obtain

$$
\lim _{\tau \rightarrow \infty} \mathrm{C}^{n}\left[\tau^{-1 / 2} \Xi_{\Psi, \tau}(f)\right]= \begin{cases}\mathrm{B}_{\Psi}^{2}\|f\|_{L^{2}}^{2} & \text { if } n=2 \\ 0 & \text { if } n \geq 3\end{cases}
$$

Taking the limit as $\tau \rightarrow 0$ is more subtle. We shall see that we recover the cumulants of the sine process given by [39], Lemma 2, and the cancellation follows from the main combinatorial lemma. For a given composition $\mathbf{m}$ of $n$ and $u \in \mathbb{R}_{0}^{n}$, by Definition (1.28),

$$
\mathrm{G}_{0}^{\mathbf{m}}(u, x)=\sum_{\sigma \in \mathbb{S}(n)} \max _{i \leq \ell}\left\{\Lambda_{i, \mathfrak{s}}^{\mathbf{m}}(u)\right\}
$$

where $\mathfrak{s}=\mathfrak{s}_{\ell(\mathbf{m})}(\sigma),(1.27)$. The important fact is that this expression becomes independent of the variable $x$. So, in the following, we will denote $G_{0}^{\mathbf{m}}(u)$ instead of $\mathrm{G}_{0}^{\mathbf{m}}(u, x)$ and we define

$$
\triangle^{\mathbf{m}}(u)=\left(u_{1}+\cdots+u_{\bar{m}_{1}}, u_{1}+\cdots+u_{\bar{m}_{2}}, \ldots, u_{1}+\cdots+u_{\bar{m}_{\ell-1}}, 0\right) .
$$

By definition (1.26), for any $u \in \mathbb{R}_{0}^{n}$, we have

$$
\Lambda_{i, s}^{\mathbf{m}}(u)= \begin{cases}u_{\bar{m}_{s}+1}+\cdots+u_{\bar{m}_{i}} & \text { if } s<i, \\ u_{\bar{m}_{s}+1}+\cdots+u_{n}+u_{1}+\cdots+u_{\bar{m}_{i}} & \text { if } i<s, \\ 0 & \text { if } i=s .\end{cases}
$$

For any $s=1, \ldots, \ell(\mathbf{m})$ we let $\pi_{s} \in \mathbb{S}(n)$ be the cyclic permutation given by

$$
\pi_{s}(i)=\bar{m}_{s}+i \bmod n .
$$

Then we see that $\left\{\Lambda_{i, s}^{\mathbf{m}}(u): i=1, \ldots, \ell\right\}=\left\{\triangle_{i}^{\mathbf{m}}\left(\pi_{s} u\right): i=1, \ldots, \ell\right\}$ and, by (4.55), we obtain

$$
\begin{aligned}
\mathrm{G}_{0}^{\mathbf{m}}(u) & =\sum_{\sigma \in \mathbb{S}(n)} \max _{i \leq \ell}\left\{\triangle_{i}^{\mathbf{m}}\left(\pi_{\mathfrak{s}} u\right)\right\}, \\
\sum_{\pi \in \mathbb{S}(n)} \mathrm{G}_{0}^{\mathbf{m}}(\pi u) & =n ! \sum_{\pi \in \mathbb{S}(n)} \max _{i \leq \ell}\left\{\triangle_{i}^{\mathbf{m}}(\pi u)\right\} .
\end{aligned}
$$


By dominated convergence, we can pass to the limit $\tau \rightarrow 0$ in formulae (4.31)(4.32). The two integrals decouple and, since $\int_{\mathbb{R}_{<}^{n}} \prod_{i=1}^{n} \Phi\left(x_{i}\right) d^{n} x=1 / n$ !, we obtain

(4.58) $\lim _{\tau \searrow 0} C^{n}\left[\Xi_{\Psi, \tau^{\prime}} f\right]=-2 \int_{\mathbb{R}_{0}^{n}} \Re\left\{\prod_{i=1}^{n} \hat{f}\left(u_{i}\right)\right\} \frac{1}{n !} \sum_{|\mathbf{m}|=n} \mathbf{M}(\mathbf{m}) \mathrm{G}_{0}^{\mathbf{m}}(u) d^{n-1} u$.

This limit is independent of the shape $\Psi$ and it will be denoted by $C^{n}\left[\Xi_{0} f\right]$. If we use the notation (4.56), Soshnikov's main combinatorial lemma reads for any $u \in \mathbb{R}_{0}^{n}$,

$$
\sum_{\pi \in \mathbb{S}(n)} \sum_{|\mathbf{m}|=n} \mathbf{M}(\mathbf{m}) \max \left\{\triangle^{\mathbf{m}}(\pi u)\right\}= \begin{cases}-\left|u_{1}\right| & \text { if } n=2 \\ 0 & \text { if } n \geq 3\end{cases}
$$

Next, we symmetrize formula (4.58) over all permutations of $u$, by equations (4.57) and (4.59), we conclude that for any $n \geq 3$,

$$
\mathrm{C}^{n}\left[\Xi_{0} f\right]=0
$$

and

$$
\mathrm{C}^{2}\left[\Xi_{0} f\right]=\int_{\mathbb{R}} \hat{f}(u) \hat{f}(-u)|u| d u .
$$

This shows that the random field $\Xi_{0}$ is Gaussian with covariance structure given by $\langle f, g\rangle_{H^{1 / 2}}$.

In the proof of Proposition 4.14, we have seen that the temperature $\tau>0$ can be treated just as a scaling parameter. Therefore, in the sequel, we will assume that $\tau=1$ and write $\Xi_{\Psi}=\Xi_{\Psi, 1}, G^{\mathbf{m}}=G_{1}^{\mathbf{m}}$, etc. By Definition 4.9, the behavior of the Poisson component of the field $\Xi_{\Psi}$ is encoded by the coefficients $\mathrm{B}_{\Psi}^{n}$, (1.29). In the remainder of this section, we will compute the generating function of the sequence $\mathrm{B}_{\Psi}^{n}$ and prove Proposition 1.9. We start by a combinatorial lemma.

Proposition 4.16. For any $z, w \in \mathbb{C}$ so that $\left|w\left(e^{(1+w) z}-1\right)\right|<|1+w|$,

$$
\sum_{n=1}^{\infty} \sum_{k=0}^{n-1} b_{k}^{n} \frac{w^{k+1} z^{n}}{n !}=\frac{w\left(e^{(1+w) z}-1\right)}{1+w e^{(1+w) z}}
$$

ProOF. By equation (1.30),

$$
b_{k}^{n}=\sum_{l=1}^{k+1}(-1)^{l+1}\left(\begin{array}{c}
n-l \\
k+1-l
\end{array}\right) \sum_{\substack{n_{1}, \ldots, n_{l} \geq 1 \\
n_{1}+\cdots+n_{l}=n}} \frac{n !}{n_{1} ! \cdots n_{l} !} .
$$


So that if we exchange the order of summation between the indices $k$ and $l$,

$$
\begin{aligned}
\sum_{n=1}^{\infty} \sum_{k=0}^{n-1} b_{k}^{n} \frac{w^{k+1} z^{n}}{n !} & =\sum_{n=1}^{\infty} z^{n} \sum_{l=1}^{n} \sum_{\substack{n_{1}, \ldots, n_{l} \geq 1 \\
n_{1}+\cdots+n_{l}=n}} \frac{(-1)^{l+1}}{n_{1} ! \cdots n_{l} !} \sum_{k=l-1}^{n-1}\left(\begin{array}{c}
n-l \\
k+1-l
\end{array}\right) w^{k+1} \\
& =\sum_{n=1}^{\infty} z^{n} \sum_{l=1}^{n} \sum_{\substack{n_{1}, \ldots, n_{l} \geq 1 \\
n_{1}+\cdots+n_{l}=n}} \frac{(-1)^{l+1}}{n_{1} ! \cdots n_{l} !} w^{l}(1+w)^{n-l}
\end{aligned}
$$

Then, since $\sum_{n=l}^{\infty} a^{n} \sum_{\substack{n_{1}, \ldots, n_{l} \geq 1 \\ n_{1}+\cdots+n_{l}=n}} \frac{1}{n_{1} ! \cdots n_{l} !}=\left(e^{a}-1\right)^{l}$ for any $a \in \mathbb{C}$, if we exchange the order of summation between $l$ and $n$, we obtain

$$
\sum_{n=1}^{\infty} \sum_{k=0}^{n-1} b_{k}^{n} \frac{w^{k+1} z^{n}}{n !}=\sum_{l=1}^{\infty}(-1)^{l+1}\left(\frac{w}{1+w}\right)^{l}\left(e^{(1+w) z}-1\right)^{l} .
$$

This proves (4.60) using the identity $\sum_{1 \leq l}(-1)^{l+1} \xi^{l}=\frac{\xi}{1+\xi}$, if $|\xi|<1$.

If we substitute $w=\frac{\Psi(x)}{1-\Psi(x)}$ and $z=\xi(1-\Psi(x))$ into (4.60), we obtain for any $|\xi|<e^{-1}$

$$
\sum_{n=1}^{\infty} \frac{\xi^{n}}{n !} \sum_{k=0}^{n-1} b_{k}^{n} \Psi(x)^{k+1}(1-\Psi(x))^{n-k-1}=\frac{\Psi(x)\left(e^{\xi}-1\right)}{1+\Psi(x)\left(e^{\xi}-1\right)} .
$$

Integrating both sides, by definition (1.29), this implies that

$$
\sum_{n=1}^{\infty} \frac{\xi^{n}}{n !} \mathrm{B}_{\Psi}^{n}=\int_{\mathbb{R}} \frac{x\left(e^{\xi}-1\right)}{1+\Psi(x)\left(e^{\xi}-1\right)} \Phi(x) d x .
$$

Motivated by Proposition 4.14, it is meaningful to raise the question: which shape $\Psi$ satisfies the conditions $\mathrm{B}_{\Psi}^{n}=0$ for all $n>2$ ? Since we assume that $\mathrm{B}_{\Psi}^{1}=$ 0 and $\mathrm{B}_{\Psi}^{2}=1$, by formula (4.61), this amounts to solving the integral equation

$$
\int_{\mathbb{R}} \frac{x\left(e^{\xi}-1\right)}{1+\Psi(x)\left(e^{\xi}-1\right)} \Phi(x) d x=\frac{\xi^{2}}{2} .
$$

Proof of Proposition 1.9. Since $(1-\Psi)$ is a cumulative distribution function, we can make the change of variable $s=\Psi(x)$ in the RHS of formula (4.61) and this leads to the identity

$$
\sum_{n=1}^{\infty} \frac{\xi^{n}}{n !} \mathrm{B}_{\Psi}^{n}=\int_{0}^{1} \frac{\Psi^{-1}(s)}{\left(e^{\xi}-1\right)^{-1}+s} d s
$$


where $\Psi^{-1}$ is the generalized inverse of $\Psi ; \Psi^{-1}(s)=\inf \{t \in \mathbb{R}: \Psi(t) \leq t\}$. If we also make the change of variable $w=\left(1-e^{\xi}\right)^{-1}$, we see that equation (4.62) gives

$$
\int_{0}^{1} \frac{\Psi^{-1}(s)}{s-w} d s=\frac{\sigma^{2}}{2}\left(\log \left(1-w^{-1}\right)\right)^{2} .
$$

Note that, since $\Psi$ is continuous, the function $\Psi^{-1}$ is continuous almost everywhere. Moreover, the conditions $\Psi \in L^{1}(0, \infty)$ and $(1-\Psi) \in L^{1}(-\infty, 0)$ guarantees that the RHS of formula (4.64) defines an analytic function in $\mathbb{C} \backslash[0,1]$. Thus, if we use the principal branch of the logarithm, the LHS of formula (4.64) is also analytic in the same domain and, by analytic continuation, equation (4.64) holds for any $w \in \mathbb{C} \backslash[0,1]$. It is well known that, for any $t \in(0,1)$ where the function $\Psi^{-1}$ is continuous,

$$
\lim _{\eta \searrow 0} \Im\left(\int_{0}^{1} \frac{\Psi^{-1}(s)}{s-(t+i \eta)} d s\right)=\pi \Psi^{-1}(t) .
$$

For the principal branch, for any $t \in(0,1)$,

$$
\lim _{\eta \searrow 0} \log \left(1-(t+i \eta)^{-1}\right)=\log \left(t^{-1}-1\right)+i \pi .
$$

Hence, by (4.64), these limits implies that if $\Psi \in \mathfrak{F}$ is solution of (4.61), then for almost all $t \in(0,1)$,

$$
\Psi^{-1}(t)=\log \left(t^{-1}-1\right) .
$$

Since, by assumption, $\Psi^{-1}$ is nonincreasing, equation (4.65) holds for all $t \in(0,1)$ and it is straightforward to see that this amounts to $\Psi(x)=1 /\left(1+e^{x}\right)$, so that the unique solution of equation (4.62) is the MNS shape $\psi$.

By Definition 4.9, Proposition 1.9 means that the Fermi statistics $\psi(t)=$ $1 /\left(1+e^{t}\right)$ is the only shape in $\mathfrak{F}$ for which the Poisson component of the field $\Xi_{\psi}$ corresponds to a Gaussian process. In general, formula (4.63) implies that Laplace transform of the Poisson component of the random process $\Xi_{\Psi}$ is given by for all $f \in C_{0}(\mathbb{R})$ and all $|\xi|<e^{-1} /\|f\|_{\infty}$,

$$
\exp \left(\sum_{n=1}^{\infty} \frac{\xi^{n}}{n !} \mathrm{B}_{\Psi}^{n} \int_{\mathbb{R}} f(t)^{n} d t\right)=\exp \left(\int_{\mathbb{R}} \int_{0}^{1} \frac{\Psi^{-1}(t)}{\left(e^{\xi f(x)}-1\right)^{-1}+t} d t d x\right) .
$$

In particular, for an arbitrary shape $\Psi \in \mathfrak{F}$, it seems very difficult to check whether the RHS of formula (4.66) defines a positive definite function in the variable $\xi$ so that the Poisson component of the field $\Xi_{\Psi}$ comes from a random process. 
4.4. Fluctuations of the critical MNS ensemble. Propositions 1.9 and 4.14 imply that for any modified Ensemble whose shape $\Psi \neq \psi$, the limiting fluctuations at the critical scale are not Gaussian. The goal of this section is to prove that this is also the case for the MNS ensemble. Our first attempt is to compute the third cumulant of the random variable $\Xi_{\psi} f$, but it turns out that it vanishes for any test function; see Proposition 4.19. Consequently, we construct a test function $y \in \mathcal{S}(\mathbb{R})$ such that $C^{4}\left[\Xi_{\psi} y\right] \neq 0$. Our strategy to simplify formula (4.31) is to symmetrize the functions $\mathrm{G}^{\mathbf{m}}$ with respect to all permutations of the variables $u_{i}$ and to look for cancellations. There are even more simplifications available using the constraints $u_{1}+\cdots+u_{n}=0$ and the DHK formulae; see Remark 4.20 below. However, as we emphasized in the Introduction, it turns out there is no counterpart of Soshnikov's main combinatorial lemma for the modified ensembles and already for the 4th cumulant, there are not enough cancellations to rewrite formula (4.31) in a simple way. Moreover, we shall only consider the following subclass of shapes.

DEFINITION 4.17. A shape $\Psi \in \mathfrak{F}$ is called symmetric if its differential $-\Phi$ is even. In other words, if it satisfies $1-\Psi(-t)=\Psi(t)$ for all $t \in \mathbb{R}$.

We can deduce from Proposition 4.16 that the triangular array $b_{k}^{n}$ satisfies for any $k=0, \ldots, n-1, b_{k}^{n}=(-1)^{n+1} b_{n-1-k}^{n}$. This implies that for any symmetric shape the map $x \mapsto \Phi(x) \sum_{k=0}^{n-1} b_{k}^{n} \Psi(x)^{k}(1-\Psi(x))^{n-1-k}$ is even when the index $n$ is odd. Thus, for any $m \geq 1$,

$$
\mathrm{B}_{\Psi}^{2 m+1}=\int_{\mathbb{R}} x \Phi(x) \sum_{k=0}^{n-1} b_{k}^{n} \Psi(x)^{k}(1-\Psi(x))^{n-1-k} d x=0 .
$$

LEMMA 4.18. We define the function $\varpi: \mathbb{R}^{2} \rightarrow \mathbb{R}^{2}$ by

$$
\varpi\left(v_{1}, v_{2}\right)=\left[v_{1}\right]^{+}+\left[v_{2}\right]^{+}+\left[v_{1}+v_{2}\right]^{+}-2 \max \left\{0, v_{1}, v_{1}+v_{2}\right\} \text {. }
$$

By formula (4.32), for any $\Psi \in \mathfrak{F}$ and any function $f \in H_{0}^{1}(\mathbb{R})$, we have

$$
\begin{aligned}
\mathrm{C}^{3}\left[\Xi_{\Psi} f\right]= & 2 \mathrm{~B}_{\Psi}^{3} \int f(x)^{3} d x+24 \int_{\mathbb{R}_{0}^{3}} d^{2} u \Re\left\{\prod_{i} \hat{f}\left(u_{i}\right)\right\} \int_{\mathbb{R}} d s \Phi(s) \\
& \times \iint_{(0, \infty)^{2}} d^{2} z \Phi\left(s+z_{1}\right) \Phi\left(s+z_{1}+z_{2}\right) \varpi\left(u_{1}-z_{1}, u_{2}-z_{2}\right),
\end{aligned}
$$

where $d^{2} u=d u_{1} d u_{2}$ and it is understood that $u_{3}=-u_{1}-u_{2}$.

Proof. See Appendix C.

The important feature of formula (4.68) is that the functions $\hat{f}$ and $\Phi$ are coupled by a function $\varpi$ which only depends on the variables $u_{i}-z_{i}$. Moreover, it follows from the DHK formula (4.69), that this function is anti-symmetric and this leads to the following result. 
PROPOSITION 4.19. If the shape $\Psi \in \mathfrak{F}$ is symmetric, then for any function $f \in H_{0}^{1}(\mathbb{R})$, we have $\mathrm{C}^{3}\left[\Xi_{\Psi} f\right]=0$.

PROOF. First, observe that by (4.67), the constant $\mathrm{B}_{\Psi}^{3}=0$ and it remains to show that the second term in formula (4.68) vanishes as well. For any $w \in \mathbb{R}^{2}$, we easily check that

(4.69) $\max \left\{0, v_{1}, v_{1}+v_{2}\right\}+\max \left\{0, v_{2}, v_{1}+v_{2}\right\}=\left[v_{1}\right]^{+}+\left[v_{2}\right]^{+}+\left[v_{1}+v_{2}\right]^{+}$.

This implies that we may express

$$
\varpi\left(v_{1}, v_{2}\right)=\max \left\{0, v_{2}, v_{1}+v_{2}\right\}-\max \left\{0, v_{1}, v_{1}+v_{2}\right\} .
$$

In particular, $\varpi\left(v_{1}, v_{2}\right)=-\varpi\left(v_{2}, v_{1}\right)$ and it follows that

$$
\begin{aligned}
& \iiint_{\mathbb{R} \times(0, \infty)^{2}} \Phi(x) \Phi\left(x+z_{1}\right) \Phi\left(x+z_{1}+z_{2}\right) \varpi\left(u_{1}-z_{1}, u_{2}-z_{2}\right) d x d^{2} z \\
& =\iiint_{\mathbb{R} \times(0, \infty)^{2}} \Phi\left(-y-z_{1}-z_{2}\right) \Phi\left(-y-z_{2}\right) \\
& \quad \times \Phi(-y) \varpi\left(u_{1}-z_{1}, u_{2}-z_{2}\right) d y d^{2} z \\
& =\iiint_{\mathbb{R} \times(0, \infty)^{2}} \Phi(y) \Phi\left(y+z_{1}\right) \Phi\left(y+z_{1}+z_{2}\right) \varpi\left(u_{1}-z_{2}, u_{2}-z_{1}\right) d y d^{2} z \\
& =-\iiint_{\mathbb{R} \times(0, \infty)^{2}} \Phi(y) \Phi\left(y+z_{1}\right) \\
& \quad \times \Phi\left(y+z_{1}+z_{2}\right) \varpi\left(u_{2}-z_{1}, u_{1}-z_{2}\right) d y d^{2} z .
\end{aligned}
$$

At first, we made the change of variable $y=-x-z_{1}-z_{2}$. In the second equality, we used the assumption that $\Phi$ is symmetric and permuted the variables $z_{1}$ and $z_{2}$. For the last equality, we used the anti-symmetry of the function $\varpi$. This shows that the integral (4.70) changes sign under permutation of the variables $u_{1}$ and $u_{2}$. Because of this fact and the symmetry of formula (4.68), the 3rd cumulant of the random variable $\Xi_{\Psi} f$ vanishes.

REMARK 4.20. The Dyson, Hunt, Kac (DHK) formulae are the following remarkable identities. For any $n \geq 2$,

$$
\begin{aligned}
& \sum_{\pi \in \mathbb{S}(n)} \max \left\{u_{\pi(1)}, u_{\pi(1)}+u_{\pi(2)}, \ldots, u_{\pi(1)}+\cdots+u_{\pi(n-1)}, 0\right\} \\
& =\sum_{\pi \in \mathbb{S}(n)} \sum_{l=1}^{n} \frac{1}{l}\left[u_{\pi(1)}+\cdots+u_{\pi(l)}\right]^{+}
\end{aligned}
$$

When $n=2$, this reduces to formula (4.69). We refer to Simon's book [36], Section 6.5, for a proof of (4.71) and an application to the strong Szegó theorem. 
Actually, the proof of Soshnikov's main combinatorial lemma (4.59) is also based on these formulae; see [26], Appendix A. We can also apply the formulae (4.71) to the cumulants of linear statistics of the modified ensembles but, except for the third cumulant, this only leads to partial simplifications.

To compute the 4 th cumulant, we make the change of variables $x \in \mathbb{R}_{<}^{4} \mapsto$ $(s, z) \in \mathbb{R} \times \mathbb{R}_{+}^{3}$ given by

$$
s=x_{1}, \quad z_{1}=x_{2}-x_{1}, \quad z_{2}=x_{3}-x_{2}, \quad z_{3}=x_{4}-x_{3},
$$

in equation (4.31). We get

$$
\begin{aligned}
\mathfrak{G}_{\psi}^{4}(f)= & -2 \int_{(0, \infty)^{3}} d^{3} z \Theta(z) \int_{\mathbb{R}_{0}^{4}} d^{3} u \Re\left\{\prod_{i=1}^{4} \hat{f}\left(u_{i}\right)\right\} \\
& \times \sum_{|\mathbf{m}|=4} \mathbf{M}(\mathbf{m}) \tilde{G}^{\mathbf{m}}(u, z),
\end{aligned}
$$

where $\tilde{\mathrm{G}}^{\mathbf{m}}(u, z)$ is the image of $x \mapsto \mathrm{G}^{\mathbf{m}}(u, x)$ under this change of variables (this function does not depend on the variable $s$ ) and

$$
\Theta(z)=\int_{\mathbb{R}} \phi(s) \phi\left(s+z_{1}\right) \phi\left(s+z_{1}+z_{2}\right) \phi\left(s+z_{1}+z_{2}+z_{3}\right) d s .
$$

It is worth noting that, since the function $\phi$ is even, we have $\Theta\left(z_{3}, z_{2}, z_{1}\right)=$ $\Theta\left(z_{1}, z_{2}, z_{3}\right)$ but no further symmetry. We are not able to obtain a compact formula for the 4th cumulant and it turns out to be simpler to compute the value of the functions $\tilde{\mathrm{G}}^{\mathbf{m}}(u, z)$ at certain well-chosen points and deduce from formula (4.72) that $\mathfrak{G}_{\psi}^{4}(y) \neq 0$ when the test function $y$ which is sufficiently concentrated around these points. The technical result that we need is summed up by Lemma C.1 which implies that

$$
\sum_{\substack{v_{1}+\ldots+v_{4}=0 \\ v_{i} \in\{-1,1\}}} \int_{(0, \infty)^{3}} \Theta(z) \sum_{|\mathbf{m}|=4} \mathbf{M}(\mathbf{m}) \tilde{G}^{\mathbf{m}}(v, z) d^{3} z=0.29 \ldots
$$

The integral can be performed analytically or numerically using Mathematica. To complete our argument, we also need the following approximation lemma. Its proof is rather straightforward and for completeness it will be given after our example.

LEMMA 4.21. Let $g(x)=e^{-\pi x^{2}}$ and

$$
\mathfrak{A}(u)=\int_{(0, \infty)^{3}} \Theta(z) \sum_{|\mathbf{m}|=4} \mathbf{M}(\mathbf{m}) \tilde{\mathrm{G}}^{\mathbf{m}}(u, z) d^{3} z .
$$


For any $v \in \mathbb{R}^{4}$, we have

$$
\lim _{\epsilon \rightarrow 0} \epsilon^{-4} \int_{\mathbb{R}_{0}^{4}} g\left(\frac{u_{1}-v_{1}}{\epsilon}\right) \cdots g\left(\frac{u_{4}-v_{4}}{\epsilon}\right) \mathfrak{A}(u) d^{3} u=\frac{\mathfrak{A}(v)}{2} \delta_{0}\left(v_{1}+\cdots+v_{4}\right) .
$$

We let $y(x)=2 e^{-\epsilon \pi x^{2}} \cos (2 \pi x)$ with $\epsilon>0$, so that

$$
\hat{y}(u)=\epsilon^{-1} g\left(\frac{u-1}{\epsilon}\right)+\epsilon^{-1} g\left(\frac{u+1}{\epsilon}\right)
$$

and by Lemma 4.21, we obtain

$$
\begin{aligned}
& \lim _{\epsilon \rightarrow 0} \int_{\mathbb{R}_{0}^{4}} d^{3} u \Re\left\{\prod_{i=1}^{4} \hat{y}\left(u_{i}\right)\right\} \int_{(0, \infty)^{3}} d^{3} z \Theta(z) \sum_{|\mathbf{m}|=4} \mathbf{M}(\mathbf{m}) \tilde{\mathbf{G}}^{\mathbf{m}}(u, z) \\
& \quad=\frac{1}{2} \sum_{\substack{v_{1}+\ldots+v_{4}=0 \\
v_{i} \in\{-1,1\}}} \mathfrak{A}(v) .
\end{aligned}
$$

The RHS of equation (4.75) is given by (4.74) and by formula (4.72), this implies that

$$
\lim _{\epsilon \rightarrow 0} \mathfrak{G}_{\psi}(y)=-0.29 \ldots
$$

Since the constant $\mathrm{B}_{\psi}^{4}=0$ by Proposition 1.9 , we conclude that, if the parameter $\epsilon$ is sufficiently small, $C^{4}\left[\Xi_{\psi} y\right] \neq 0$ and the linear statistics $\Xi_{\psi} y$ is not Gaussian.

Proof OF Lemma 4.21. Let us fix $v \in \mathbb{R}^{4}$ and let $r(v)=v_{1}+\cdots+v_{4}$. It is easy to see that the functions $u \mapsto \tilde{G}^{\mathbf{m}}(u, z)$ are Lipchitz continuous with respect to $|u|_{\infty}$ with constants which can be chosen independently of $z \in \mathbb{R}_{+}^{3}$. Then the function $\mathfrak{A}(u)$ is also Lipschitz continuous on $\mathbb{R}^{3}$ and a change of variables yields

$$
\begin{aligned}
\epsilon^{-4} & \int_{\mathbb{R}_{0}^{4}} g\left(\frac{u_{1}-v_{1}}{\epsilon}\right) \cdots g\left(\frac{u_{4}-v_{4}}{\epsilon}\right) \mathfrak{A}(u) d^{3} u \\
= & \int_{\mathbb{R}_{-r(v) / \epsilon}^{4}} g\left(w_{1}\right) \cdots g\left(w_{4}\right) \mathfrak{A}(v+\epsilon w) d^{3} w \\
& =\mathfrak{A}(v) \int_{\mathbb{R}_{-r(v) / \epsilon}^{4}} g\left(w_{1}\right) \cdots g\left(w_{4}\right) d^{3} w+\underset{\epsilon \rightarrow 0}{O}(\epsilon) .
\end{aligned}
$$

If we let $X_{\epsilon} \in \mathcal{N}\left(\epsilon^{-1} r(v), \frac{3}{2 \pi}\right)$, it is easy to see that

$$
\int_{\mathbb{R}_{-r(v) / \epsilon}^{4}} g\left(w_{1}\right) \cdots g\left(w_{4}\right) d^{3} w=\mathbb{E}\left[e^{-\pi X_{\epsilon}^{2}}\right] .
$$


So that if $r(v)=v_{1}+\cdots+v_{4}=0$, then $\mathbb{E}\left[e^{-\pi X_{\epsilon}^{2}}\right]=\frac{1}{2}$ for any $\epsilon>0$ and it follows that

$$
\lim _{\epsilon \rightarrow 0} \epsilon^{-4} \int_{\mathbb{R}_{0}^{4}} g\left(\frac{u_{1}-v_{1}}{\epsilon}\right) \cdots g\left(\frac{u_{4}-v_{4}}{\epsilon}\right) \mathfrak{A}(u) d^{4} u=\frac{1}{2} \mathfrak{A}(v) .
$$

On the other hand if $r(v) \neq 0$, by equation (4.76),

$$
\lim _{\epsilon \rightarrow 0} \int_{\mathbb{R}_{r(v) / \epsilon}^{4}} g\left(w_{1}\right) \cdots g\left(w_{4}\right) d^{4} w=0 .
$$

\section{APPENDIX A: ASYMPTOTICS OF THE HERMITE POLYNOMIALS}

In this section, we provide some background on the asymptotics of the Hermite polynomials and the GUE kernel; see, for instance, [30], Proposition 5.1.3, or [12], Section 2.2. These asymptotics are named after Plancherel-Rotach, [31], and can be derived using the classical saddle point method. To investigate the statistics of Hermitian invariant ensembles, one is usually interested in uniform asymptotics of the Christoffel-Darboux kernels. Based on the Riemann-Hilbert problem for orthogonal polynomials, the sine-kernel asymptotics have been established in [13] at the microscopic scale for a large class of potentials. These results have recently been extended to mesoscopic scales in [24] (see also [25]), and we will briefly review the case of the GUE kernel. Let $h_{n}$ be the normalized Hermite polynomial of degree $n \in \mathbb{N}$ with respect to $e^{-x^{2}}$ on $\mathbb{R}$ and define the Hermite functions:

$$
\vartheta_{n}(x)=h_{n}(x) e^{-\frac{x^{2}}{2}} .
$$

Definition A.1. We define on $[-1,1]$ the functions $\varrho(t)=2 \sqrt{1-t^{2}}$ and

$$
F(x)=\int_{0}^{x} \varrho(t) d t=\arcsin (x)+x \sqrt{1-x^{2}} .
$$

The map $F$ is a diffeomorphism from $|x|<1$ to $|x|<\frac{\pi}{2}$ and we let $G$ be its inverse.

The Hermite polynomials have the following bulk asymptotics:

$$
\begin{aligned}
\vartheta_{n}(\sqrt{2 n} x)= & \left(\frac{2}{n\left(1-x^{2}\right)}\right)^{1 / 4} \frac{1}{\sqrt{\pi}} \\
& \times\left\{\cos \left(n \frac{\pi}{2}-n F(x)-\frac{1}{2} \arcsin (x)\right)+\underset{n \rightarrow \infty}{O}\left(\beta^{-3 / 2} n^{-3 \gamma / 2}\right)\right\},
\end{aligned}
$$

for all $|x| \leq 1-\beta n^{-2 / 3+\gamma}$ where $0<\gamma \leq 2 / 3$ and $0<\beta<1$. Observe that, in the case $\gamma=2 / 3$, we obtain an asymptotics valid in any fixed interval $[-1+$ 
$\beta, 1-\beta]$ with a uniform error term of order $1 / n$. While the Hermite functions have oscillatory behavior inside the bulk, they have exponential decay outside:

$$
\vartheta_{n}(\sqrt{2 n} x)=\frac{e^{-n H(x)}\left\{1+O\left(n^{-3 \gamma / 2}\right)\right\}}{\sqrt{\pi \sqrt{2 n} \sqrt{x^{2}-1}}},
$$

for all $|x| \geq 1+n^{-2 / 3+\gamma}$ where $0<\gamma<2 / 3$ and the function $H$ is even and defined for any $x>1$ by

$$
H(x)=\int_{1}^{x} 2 \sqrt{t^{2}-1} d t=x \sqrt{x^{2}-1}-\log \left(x+\sqrt{x^{2}-1}\right) .
$$

In particular, since $\frac{4 \sqrt{2}}{3}(x-1)^{3 / 2} \leq H(x)$, there exist a constants $C>0$ such that for any $x>1+n^{-1 / 2}$,

$$
\left|\vartheta_{n}(\sqrt{2 n} x)\right| \leq C e^{-n \frac{4 \sqrt{2}}{3}(x-1)^{3 / 2}} .
$$

At the edge, the asymptotics is also well known but, since we are only interested in bulk linear statistics in this paper, we will not need any precise estimate and use instead the uniform bound

$$
\left\|\vartheta_{n}\right\|_{\infty} \leq c_{H} n^{-1 / 12}
$$

where $c_{H}$ is a universal constant and the exponent is sharp. We define the Christoffel-Darboux kernel:

$$
K_{\mathrm{CD}}^{N}(x, y)=\sum_{n=0}^{N-1} \vartheta_{n}(x) \vartheta_{n}(y)=\sqrt{\frac{N}{2}} \frac{\vartheta_{N}(x) \vartheta_{N-1}(y)-\vartheta_{N-1}(x) \vartheta_{N}(y)}{x-y}
$$

and the Wigner semicircle law, for any $|t| \leq \sqrt{2}$,

$$
\varrho_{\mathrm{sc}}(t)=\frac{1}{\pi} \sqrt{2-t^{2}}=\frac{1}{\pi \sqrt{2}} \varrho\left(\frac{t}{\sqrt{2}}\right) .
$$

At the microscopic scale, it is well known that we get the sine kernel in the limit for any $\left|x_{0}\right|<\sqrt{2}$,

$$
\lim _{N \rightarrow \infty} \frac{\pi}{\sqrt{2 N}} K_{\mathrm{CD}}^{N}\left(\sqrt{N} x_{0}+\frac{\pi \xi}{\sqrt{2 N}}, \sqrt{N} x_{0}+\frac{\pi \zeta}{\sqrt{2 N}}\right)=\frac{\sin \left[\pi \varrho_{\mathrm{sc}}\left(x_{0}\right)(\xi-\zeta)\right]}{\pi(\xi-\zeta)} .
$$

Using the results of [13], these asymptotics can be extended to mesoscopic scales. In the sequel, $L$ is an arbitrary large positive constant and $\left|x_{0}\right|<\sqrt{2}$.

THEOREM A.2. For any $-1 / 2 \leq \lambda<1 / 2$, we have

$$
\begin{aligned}
& N^{\lambda} K_{\mathrm{CD}}^{N}\left(\sqrt{N} x_{0}+\xi N^{\lambda}, \sqrt{N} x_{0}+\zeta N^{\lambda}\right) \\
& \quad=\frac{\sin \left[N\left(F\left(\frac{x_{0}+N^{-1 / 2+\lambda} \xi}{\sqrt{2}}\right)-F\left(\frac{x_{0}+N^{-1 / 2+\lambda} \zeta}{\sqrt{2}}\right)\right)\right]}{\pi(\xi-\zeta)}+\underset{N \rightarrow \infty}{O}\left(N^{-1 / 2+\lambda}\right)
\end{aligned}
$$

uniformly for all $\xi, \zeta \in[-L, L]$. 
Theorem A.2 was first proved in [24] using the Riemann-Hilbert formulation of [13]. In [25], we produce an elementary proof which is based on the classical steepest descent method performed in the seminal paper [31]. Note that this approximation takes into account the density of the Wigner semicircle law, that is, the fact that the GUE eigenvalues are not uniformly distributed at the global scale. Namely, by Definition A.1 and (A.7), Theorem A.2 can be rephrased as

$$
\begin{aligned}
N^{\lambda} K_{\mathrm{CD}}^{N}\left(\sqrt{N} x_{0}+\xi N^{\lambda}, \sqrt{N} x_{0}+\zeta N^{\lambda}\right) \\
\quad=\frac{\sin \left[\pi N^{1 / 2+\lambda} \int_{\zeta}^{\xi} \varrho_{\mathrm{sc}}\left(x_{0}+t N^{-1 / 2+\lambda}\right) d t\right]}{\pi(\xi-\zeta)}+\underset{N \rightarrow \infty}{O}\left(N^{-1 / 2+\lambda}\right) .
\end{aligned}
$$

Note that unlike the sine-kernel, the RHS of (A.8) is not translation-invariant. This raises complications to compute the limits of the cumulants of large scale linear statistics of the modified GUEs; cf. Proposition 4.11. However, at sufficiently small scales, we recover the sine-kernel as a special case of Theorem A.2.

THEOREM A.3. For any $-1 / 2 \leq \lambda<0$, we have the asymptotic formula

$$
\begin{aligned}
& N^{\lambda} K_{\mathrm{CD}}^{N}\left(\sqrt{N} x_{0}+\xi N^{\lambda}, \sqrt{N} x_{0}+\zeta N^{\lambda}\right) \\
& \quad=\frac{\sin \left(N^{1 / 2+\lambda} \pi \varrho_{\mathrm{sc}}\left(x_{0}\right)(\xi-\zeta)\right)}{\pi(\xi-\zeta)}+\underset{N \rightarrow \infty}{O}\left(N^{2 \lambda}\right)
\end{aligned}
$$

uniformly for all $\xi, \zeta \in[-L, L]$.

Proof. By Definition A.1, we have

$$
F(x)-F(y)=\varrho\left(\frac{x+y}{2}\right)(x-y)+O\left(|x-y|^{3}\right) .
$$

If we let $x_{N}=\frac{x_{0}+N^{-1 / 2+\lambda} \xi}{\sqrt{2}}$ and $y_{N}=\frac{x_{0}+N^{-1 / 2+\lambda} \zeta}{\sqrt{2}}$, a Taylor expansion gives

$$
\varrho\left(\frac{x_{N}+y_{N}}{2}\right)=\varrho\left(\frac{x_{0}}{\sqrt{2}}\right)+O\left(N^{-1 / 2+\lambda}\right)
$$

and it follows that

$$
F\left(x_{N}\right)-F\left(y_{N}\right)=\frac{1}{\sqrt{2}} \varrho\left(\frac{x_{0}}{\sqrt{2}}\right)(\xi-\zeta) N^{-1 / 2+\lambda}+O\left(|\xi-\zeta| N^{-1+2 \lambda}\right) .
$$

Hence, by (A.7), we have proved that

$$
\begin{aligned}
& \sin \left[N\left(F\left(\frac{x_{0}+N^{-1 / 2+\lambda} \xi}{\sqrt{2}}\right)-F\left(\frac{x_{0}+N^{-1 / 2+\lambda} \zeta}{\sqrt{2}}\right)\right)\right] \\
& \quad=\sin \left[N^{1 / 2+\lambda} \pi \varrho_{\mathrm{sc}}\left(x_{0}\right)(\xi-\zeta)\right]+\underset{N \rightarrow \infty}{O}\left(|\xi-\zeta| N^{2 \lambda}\right) .
\end{aligned}
$$

When $\lambda<0$, the error term is converging to 0 for any $x, y \in[-L, L]$, and if we plug this approximation in the formula of Theorem A.2, we obtain the asymptotics of Theorem A.3. 
REMARK A.4. In the special case $x_{0}=0$, since $\varrho^{\prime}(0)=0$, the error term in (A.9) is of order $N^{-1+2 \lambda}$ and the sine kernel approximation of Theorem A.3 is actually valid in the whole range $-1 / 2 \leq \lambda<1 / 6$.

Note that the Christoffel-Darboux kernel $K_{\mathrm{CD}}^{N}$ is the same, up to a scaling, as the GUE kernel $K_{0}^{N}$ defined by (1.4) in the Introduction. Namely, if we let $\lambda=\frac{1}{2}-\delta$ for $\delta \in(0,1]$, by (A.6), we can rewrite

$$
N^{-\delta} K_{0}^{M}\left(x N^{-\delta}, y N^{-\delta}\right)=\frac{\pi}{\sqrt{2}} N^{\lambda} K_{\mathrm{CD}}^{M}\left(\frac{\pi}{\sqrt{2}} x N^{\lambda}, \frac{\pi}{\sqrt{2}} y N^{\lambda}\right) .
$$

Thus, for any $\epsilon>0$, if $x_{0}=0$ and $M=N+k$ for some $|k| \leq N^{1-\epsilon}$, by Theorem A.2, for all $x, y \in[-L, L]$, we have

$$
\begin{aligned}
N^{-\delta} & K_{0}^{M}\left(x N^{-\delta}, y N^{-\delta}\right) \\
& =\frac{\sin \left[M\left(F\left(\frac{\pi}{2} \frac{x}{\sqrt{M} N^{\delta-1 / 2}}\right)-F\left(\frac{\pi}{2} \frac{y}{\sqrt{M} N^{\delta-1 / 2}}\right)\right)\right]}{\pi(x-y)}+\underset{N \rightarrow \infty}{O}\left(N^{-\delta}\right) .
\end{aligned}
$$

This formula holds at any mesoscopic scales. On the other hand, if we assume that $1 / 3<\delta \leq 1$ (cf. Remark A.4), since $\varrho_{\mathrm{sc}}(0)=\frac{\sqrt{2}}{\pi}$, by Theorem A.3, we obtain the following asymptotics:

$$
\begin{aligned}
N^{-\delta} K_{0}^{M}\left(x N^{-\delta}, y N^{-\delta}\right)= & \frac{\sin \left[\pi N^{1 / 2-\delta} \sqrt{M}(x-y)\right]}{\pi(x-y)} \\
& +\underset{N \rightarrow \infty}{O}\left(N^{1-3 \delta}\right)
\end{aligned}
$$

\section{APPENDIX B: VARIANCE IN THE CRITICAL REGIME}

We give two different proofs of Theorem 1.6. First, we use the ideas of Section 3.1 to compute the limit of the reproducing variance $V_{0}\left(f_{\delta}\right)$ at the critical scale $\delta=\alpha$; see Proposition B.1. Second, we may use (4.48) and compute $C^{2}\left[\Xi_{\Psi, \tau / 4} f\right]$ using formula (1.31). Subsequently, we check that the different formulae for the critical variance are consistent and we apply them to the MNS ensemble.

Proposition B.1. For any shape $\Psi \in \mathfrak{E}$, any test function $f \in H_{0}^{1 / 2}(\mathbb{R})$ and for any scale $0<\alpha<1$, we have

$$
\lim _{N \rightarrow \infty} V_{0}\left(f_{\alpha}\right)=\frac{1}{4 \pi^{2}} \iint\left|\frac{f(x)-f(y)}{x-y}\right|^{2}\left|\hat{\Phi}\left(\frac{x-y}{4 \tau^{-1}}\right)\right|^{2} d x d y .
$$

PROOF. By formula (3.1) combined to the estimate (3.16) and the approximation (4.38), we see that if the support of the test function is included in $\left[-\frac{L}{2}, \frac{L}{2}\right]$ 
and $\Gamma_{N}=(\log N)^{2}$, then

$$
\begin{aligned}
V_{0}\left(f_{\alpha}\right)= & \frac{1}{2 \pi^{2} \tau^{2} N^{2 \alpha}} \sum_{\substack{|k| \leq \Gamma_{N} N^{\alpha} \\
|j| \leq \Gamma_{N} N^{\alpha}}} \Phi\left(\frac{k}{\tau N^{\alpha}}\right) \Phi\left(\frac{j}{\tau N^{\alpha}}\right) \iint_{[-L, L]^{2}}\left|\frac{f(x)-f(y)}{x-y}\right|^{2} \\
& \times \mathcal{S}_{k}^{N}(x, y) \mathcal{S}_{j}^{N}(x, y) d x d y+\underset{L \rightarrow \infty}{O}\left(L^{-1}\right)+\underset{N \rightarrow \infty}{O}\left(N^{-\alpha}\right),
\end{aligned}
$$

where $\mathcal{S}_{k}^{N}(x, y)=\sin \left[\sqrt{N(N+k)}\left(F\left(\frac{\pi}{2} \frac{x}{N^{\alpha}}\right)-F\left(\frac{\pi}{2} \frac{y}{N^{\alpha}}\right)\right)\right]$. Applying a trigonometric identity and the Riemann-Lebesgue lemma like in the proof of Lemma 3.2, we see that as $N \rightarrow \infty$,

$$
\begin{aligned}
V_{0}\left(f_{\alpha}\right) \simeq & \frac{1}{4 \pi^{2}} \frac{1}{\tau^{2} N^{2 \alpha}} \sum_{\substack{|k| \leq \Gamma_{N} N^{\alpha} \\
|j| \leq \Gamma_{N} N^{\alpha}}} \Phi\left(\frac{k}{\tau N^{\alpha}}\right) \Phi\left(\frac{j}{\tau N^{\alpha}}\right) \iint_{[-L, L]^{2}}\left|\frac{f(x)-f(y)}{x-y}\right|^{2} \\
& \times \cos \left(\frac{(k-j) \pi(x-y)}{2 N^{\alpha}}\right) d x d y+\underset{L \rightarrow \infty}{O}\left(L^{-1}\right) .
\end{aligned}
$$

Finally, the sums converge to Riemann integrals and by the dominated convergence theorem,

$$
\begin{gathered}
\left.\limsup _{N \rightarrow \infty}\left|V_{0}\left(f_{\alpha}\right)-\frac{1}{4 \pi^{2}} \iint_{[-L, L]^{2}}\right| \frac{f(x)-f(y)}{x-y}\right|^{2} \iint_{\mathbb{R}^{2}} \Phi(u) \Phi(v) \\
\quad \times \cos \left[\frac{\pi(u-v)(x-y)}{2 \tau^{-1}}\right] d u d v d x d y \mid \leq \frac{C(f)}{L},
\end{gathered}
$$

where the constant $C(f)$ depends only on the test function $f$ by (3.16). To complete the proof, we use that for any $w \in \mathbb{R}$,

$$
\iint_{\mathbb{R}^{2}} \Phi(u) \Phi(v) \cos [2 \pi(u-v) w] d u d v=|\hat{\Phi}(w)|^{2},
$$

and let $L \rightarrow \infty$ in the inequality (B.1).

As a consequence of Lemma 3.1, Lemma 3.2 and Proposition B.1, we obtain that for any $0<\alpha<1$ and $f \in H_{0}^{1 / 2} \cap L^{\infty}(\mathbb{R})$,

$$
\begin{aligned}
\lim _{N \rightarrow \infty} & \operatorname{Var}_{K_{\Psi, \alpha}^{N}}\left[f_{\alpha}\right] \\
\quad= & \frac{\tau}{2} \int_{\mathbb{R}} f(x)^{2} d x+\frac{1}{4 \pi^{2}} \iint_{\mathbb{R}^{2}}\left|\frac{f(x)-f(y)}{x-y}\right|^{2}\left|\hat{\Phi}\left(\frac{(x-y)}{4 \tau^{-1}}\right)\right|^{2} d x d y .
\end{aligned}
$$

By Theorem 1.7, this implies that for any $\Psi \in \mathfrak{E}$ so that $\mathrm{B}_{\Psi}^{2}=1$ and $\tau>0$,

$$
\begin{aligned}
\operatorname{Var}\left[\Xi_{\Psi, \tau} f\right]= & 2 \tau \int_{\mathbb{R}} f(x)^{2} d x \\
& +\frac{1}{4 \pi^{2}} \iint_{\mathbb{R}^{2}}\left|\frac{f(x)-f(y)}{x-y}\right|^{2}|\hat{\Phi}(\tau(x-y))|^{2} d x d y .
\end{aligned}
$$


REMARK B.2. Such a direct computation seems possible only for the variance of linear statistics thanks to the special structure of the reproducing variance $V_{0}$, (3.1). Because of the singularity of the correlation kernel $K_{\Psi, \alpha}^{N}$, it is technically involved to compute the limit of the higher-order cumulants using similar argument. Therefore, it is better to exploit instead the fact that the kernel $L_{\Psi, \eta}^{N}$ given by (1.33) is translation-invariant and use Soshnikov's method.

We have seen in Section 4.2 that, up to a scaling, the modified ensembles have the same limit at the critical scale. Thus, we have three expressions for the variance of the random variable $\Xi_{\Psi, \tau} f ;(1.31)$ and formulae (2.11) and (B.3) in the circular and Gaussian case, respectively. Note that these formulae are well defined for any shape $\Psi \in \mathfrak{F}$ and and we will now check that they are consistent. By (1.18), $\mathrm{M}(\mathbf{1}+\mathbf{1})=-1$ and by (1.26), for any $u \in \mathbb{R}_{0}^{2}$,

$$
\Lambda^{\mathbf{1}^{2}}(u)=\left(\begin{array}{cc}
0 & -u_{2} \\
u_{2} & 0
\end{array}\right)
$$

Moreover, by definition (1.28), for any $x_{1}<x_{2}$,

$$
\mathrm{G}_{\tau}^{\mathbf{1}^{2}}(u, x)=\max \left\{0, u_{2}-\tau\left(x_{2}-x_{1}\right)\right\}+\max \left\{-u_{2}-\tau\left(x_{2}-x_{1}\right), 0\right\} .
$$

Hence, for any bounded function $f \in H_{0}^{1 / 2}$, by formula (1.31) with $n=2$,

$$
\begin{aligned}
& \operatorname{Var}\left[\Xi_{\Psi, \tau} f\right] \\
& \text { (B.4) }=2 \tau \int_{\mathbb{R}} f(x)^{2} d x \\
& \quad+4 \int_{0}^{\infty}|\hat{f}(u)|^{2} \iint_{x_{1}<x_{2}} \Phi\left(x_{1}\right) \Phi\left(x_{2}\right)\left[u-\tau\left(x_{2}-x_{1}\right)\right]^{+} d u d x_{1} d x_{2} .
\end{aligned}
$$

Let us first check that this formula matches with the RHS of (2.11). Using that $\Phi=-\Psi^{\prime}$ and the properties of the shape $\Psi$, (1.5), some integrations by parts shows that for any $u>0$,

$$
\int_{\mathbb{R}} \Phi(t) \int_{0}^{\infty} \Phi(t+s)[u-s]^{+} d s d t=\frac{u}{2}-\mathrm{B}_{\Psi}^{2}+\int_{\mathbb{R}} \Psi(x+u)(1-\Psi(x)) d x .
$$

Since, by convention $\mathrm{B}_{\Psi_{\tau}}^{2}=\tau$ (cf. Remark 1.2), this implies that

$$
\begin{aligned}
\operatorname{Var}\left[\Xi_{\Psi, \tau} f\right]= & 2 \int_{0}^{\infty}|\hat{f}(u)|^{2} u d u \\
& +4 \int_{0}^{\infty}|\hat{f}(u)|^{2} \int_{\mathbb{R}} \Psi_{\tau}(x+u)\left(1-\Psi_{\tau}(x)\right) d x d u .
\end{aligned}
$$

Moreover, it is easy to check by differentiating with respect to $u \in \mathbb{R}$ that for any shape $\Psi \in \mathfrak{F}$, we have

$$
\int_{\mathbb{R}} \Psi(x)(1-\Psi(x+u)) d x=u+\int_{\mathbb{R}} \Psi(x+u)(1-\Psi(x)) d x
$$


so that, according to formula (B.5), we obtain

$$
\operatorname{Var}\left[\Xi_{\Psi, \tau} f\right]
$$

$$
=2 \int_{0}^{\infty}|\hat{f}(u)|^{2} \int_{\mathbb{R}}\left(\Psi\left(\frac{x-u}{\tau}\right)+\Psi\left(\frac{x+u}{\tau}\right)\right)\left(1-\Psi\left(\frac{x}{\tau}\right)\right) d x d u .
$$

By (2.10), this establishes that the RHS of formulae (B.4) and (2.11) are equals. It remains to check that formula (B.4) also matches with (B.3). To do so, we will need the following result.

LEMMA B.3. For any function $f \in H^{1 / 2}(\mathbb{R})$ and any $w>0$, we have

$$
\frac{1}{4 \pi^{2}} \iint_{\mathbb{R}^{2}}\left|\frac{f(x)-f(y)}{x-y}\right|^{2} \cos (2 \pi(x-y) w) d x d y=\int_{\mathbb{R}}|\hat{f}(u)|^{2}[|u|-w]^{+} d u .
$$

PROOF. By Plancherel's formula, for any $z \in \mathbb{R}$,

$$
\int_{\mathbb{R}}|f(x)-f(x+z)|^{2} d x=4 \int_{\mathbb{R}}|\hat{f}(u)|^{2} \sin ^{2}(\pi u z) d u .
$$

Then, by Fubini's theorem, for any $w>0$,

$$
\begin{aligned}
& \frac{1}{4 \pi^{2}} \iint\left|\frac{f(x)-f(y)}{x-y}\right|^{2} \cos (2 \pi(x-y) w) d x d y \\
& \quad=\frac{1}{4 \pi^{2}} \iint\left|\frac{f(x)-f(x+z)}{z}\right|^{2} \cos (2 \pi z w) d x d z \\
& \quad=\frac{1}{\pi^{2}} \int|\hat{f}(u)|^{2}\left(\int \frac{\sin ^{2}(\pi u z)}{z^{2}} \cos (2 \pi z w) d z\right) d u .
\end{aligned}
$$

Moreover, by Residue calculus, one can show that for any $w>0$ and $u \in \mathbb{R}$,

$$
\int \frac{\sin ^{2}(\pi u z)}{z^{2}} \cos (2 \pi w z) d z=\pi^{2}[|u|-w]^{+} .
$$

Hence, the lemma follows by combining equations (B.7) and (B.8).

Lemma B.3 implies that

$$
\begin{aligned}
& \frac{1}{4 \pi^{2}} \iint_{\mathbb{R}^{2}} \iint_{t<s}\left|\frac{f(x)-f(y)}{x-y}\right|^{2} \Phi(t) \Phi(s) \cos [2 \pi \tau(t-s)(x-y)] d x d y d s d t \\
& \quad=2 \int_{0}^{\infty}|\hat{f}(u)|^{2} \iint_{t<s} \Phi(t) \Phi(s)[u-\tau(s-t)]^{+} d u d s d t .
\end{aligned}
$$


Then, if we take $w=\tau(s-t)$ in formula (B.2), by symmetry we obtain

$$
\begin{aligned}
& \frac{1}{4 \pi^{2}} \iint_{\mathbb{R}^{2}}\left|\frac{f(x)-f(y)}{x-y}\right|^{2}|\hat{\Phi}(\tau(x-y))|^{2} d x d y \\
& \quad=4 \int_{0}^{\infty}|\hat{f}(u)|^{2} \iint_{t<s} \Phi(t) \Phi(s)[u-\tau(s-t)]^{+} d u d s d t
\end{aligned}
$$

Hence, if we add up the Poisson contribution $2 \tau\|f\|_{L^{2}}^{2}$, we conclude that the RHS of formulae (B.3) and (B.4) are also equals. As an example, let us see what these formulae look like for the MNS ensemble. The MNS shape is $\psi(t)=$ $\left(1+e^{t}\right)^{-1}$ and an elementary integration gives for any $u \in \mathbb{R}$,

$$
\int_{\mathbb{R}} \psi(t+u)(1-\psi(t)) d t=e^{u} \int_{0}^{\infty} \frac{1}{(1+s)\left(s+e^{u}\right)} d s=\frac{u}{1-e^{-u}} .
$$

Then, by formula (B.6), we get

$$
\operatorname{Var}\left[\Xi_{\psi, \tau} f\right]=\int_{\mathbb{R}}|\hat{f}(u)|^{2} \frac{u}{\tanh \left(\frac{u}{2 \tau}\right)} d u .
$$

We can deduce the dual of this formula using equation (B.3). We have

$$
\phi(t)=\frac{4}{\cosh [t / 2]^{2}} \quad \text { and } \quad \hat{\phi}(u)=\frac{2 \pi^{2} u}{\sinh \left[2 \pi^{2} u\right]},
$$

so that

$$
\begin{aligned}
\operatorname{Var}\left[\Xi_{\psi, \tau} f\right]= & 2 \tau \int f(x)^{2} d x \\
& +\iint|f(x)-f(y)|^{2}\left(\frac{\pi \tau}{\sinh \left[2 \pi^{2} \tau(x-y)\right]}\right)^{2} d x d y .
\end{aligned}
$$

\section{APPENDIX C: THE THIRD AND FOURTH CUMULANTS}

To prove Lemmas 4.18 and C.1 below, the strategy is to exploit the symmetries of formula (4.31) in order to simplify as much as possible the cumulants of the random variable $\Xi_{\Psi} f$. To this end, we will use the following convention. Given two functions, we write $f \equiv g$ if there exists a permutation $\sigma \in \mathbb{S}(n)$ such that $f(u)=g(\sigma u)$ or if $f(u)=g(-u)$ for all $u \in \mathbb{R}^{n}$. For any vector $\left(u_{i}\right)_{i=1}^{n}$ of real numbers, we also denote

$$
\max \left\{u_{1}, \ldots, u_{n}\right\}=\max \left\{0, u_{1}, \ldots, u_{n}\right\} .
$$

Unfortunately, the combinatorial structure behind the cumulants of the modified ensembles seems to be rather involved, and consequently the following computations are rather technical. 
PRoOf OF LEMma 4.18. According to definition (1.18), we have $\mathrm{M}(\mathbf{2}+\mathbf{1})=$ $-\frac{3}{2}, \mathbf{M}\left(\mathbf{1}^{3}\right)=2$, and by formula (4.31),

$$
\mathfrak{G}_{\Psi}^{3}[f]=\int_{\mathbb{R}_{0}^{3}} d^{2} u \Re\left\{\prod_{i=1}^{n} \hat{f}\left(u_{i}\right)\right\} \int_{\mathbb{R}_{<}^{3}} d^{3} x \prod_{i=1}^{n} \Phi\left(x_{i}\right)
$$

$$
\times \sum_{|\mathbf{m}|=n}\left\{3 \mathrm{G}^{\mathbf{2}+\mathbf{1}}(u, x)+3 \mathrm{G}^{\mathbf{1 + 2}}(u, x)-4 \mathrm{G}^{\mathbf{1}^{3}}(u, x)\right\} .
$$

Hence, to prove formula (4.68), we need only to compute the kernels $\mathrm{G}^{\mathbf{1 + 2}}$ and $\mathrm{G}^{\mathbf{1}^{3}}$. By definition (1.26), for any $u \in \mathbb{R}_{0}^{n}$,

$$
\Lambda^{\mathbf{2 + 1}}(u)=\left(\begin{array}{cc}
0 & -u_{3} \\
u_{3} & 0
\end{array}\right) \equiv\left(\begin{array}{cc}
0 & -u_{1} \\
u_{1} & 0
\end{array}\right)=-\Lambda^{\mathbf{1 + 2}}(u) .
$$

Then, by definition (1.28),

$$
\mathrm{G}^{2+1}(u, x) \equiv \mathrm{G}^{1+2}(u, x)
$$

$$
\begin{aligned}
\equiv & {\left[u_{1}-x_{2}+x_{1}\right]^{+}+\left[u_{1}-x_{3}+x_{1}\right]^{+}+\left[-u_{1}-x_{2}+x_{1}\right]^{+} } \\
& +\left[-u_{1}-x_{3}+x_{1}\right]^{+}+\left[u_{1}-x_{3}+x_{2}\right]^{+}+\left[-u_{1}-x_{3}+x_{2}\right]^{+} \\
\equiv & 2\left(\left[u_{1}-x_{2}+x_{1}\right]^{+}+\left[u_{1}+u_{2}-x_{3}+x_{1}\right]^{+}+\left[u_{2}-x_{3}+x_{2}\right]^{+}\right) .
\end{aligned}
$$

One can check that each term of the first line corresponds to a permutation in $\mathbb{S}(3)$ in the following order 123,132, 213,312, 231,321. At the second step, we used the symmetries and the condition $u_{1}+u_{2}+u_{3}=0$. By a similar argument, the contribution of the composition $\mathbf{1}^{3}=(1,1,1)$ is given by

$$
\Lambda^{\mathbf{1}^{3}}(u)=\left(\begin{array}{ccc}
0 & -u_{2} & -u_{2}-u_{3} \\
u_{2} & 0 & -u_{3} \\
u_{2}+u_{3} & u_{3} & 0
\end{array}\right)
$$

and

$$
\begin{aligned}
\mathrm{G}^{1^{3}}(u, x)= & \stackrel{+}{\operatorname{ax}}\left\{u_{2}-x_{2}+x_{1}, u_{2}+u_{3}-x_{3}+x_{1}\right\} \\
& +\stackrel{+}{\max }\left\{u_{2}-x_{3}+x_{1}, u_{2}+u_{3}-x_{2}+x_{1}\right\} \\
& +\underset{+}{+} \max \left\{-u_{2}-x_{2}+x_{1}, u_{3}-x_{3}+x_{1}\right\} \\
& +\max ^{+}\left\{-u_{2}-x_{3}+x_{1}, u_{3}-x_{2}+x_{1}\right\} \\
& +\max ^{+}\left\{-u_{2}-u_{3}-x_{2}+x_{1},-u_{3}-x_{3}+x_{1}\right\} \\
& +\underset{+}{+}\left\{-u_{2}-u_{3}-x_{3}+x_{1},-u_{3}-x_{2}+x_{1}\right\} .
\end{aligned}
$$

It is straightforward to see that the previous formula implies that

$$
\mathrm{G}^{\mathbf{1}^{3}}(u, x) \equiv 6 \underset{\max }{+}\left\{u_{1}-x_{2}+x_{1}, u_{1}+u_{2}-x_{3}+x_{1}\right\} .
$$


Then, if we combine formulae (C.2) and (C.3), we get

$$
\begin{array}{r}
3 \mathrm{G}^{\mathbf{2}+\mathbf{1}}(u, x)+3 \mathrm{G}^{\mathbf{1 + 2}}(u, x)-4 \mathrm{G}^{\mathbf{1}^{3}}(u, x) \\
\equiv 12 \varpi\left(u_{1}-x_{2}+x_{1}, u_{2}-x_{3}+x_{2}\right) .
\end{array}
$$

Finally, if we make the change of variables $x_{1}=x, z_{1}=x_{2}-x_{1}$ and $z_{2}=x_{3}-x_{2}$ in (C.1), formula (C.4) implies that

$$
\begin{aligned}
\mathrm{C}^{3}\left[\Xi_{\Psi} f\right]= & 12 \int_{\mathbb{R}^{2}} d^{2} u \Re\left\{\prod_{i} \hat{f}\left(u_{i}\right)\right\} \int_{\mathbb{R}} d x \Phi(x) \\
& \times \iint_{(0, \infty)^{2}} \Phi\left(x+z_{1}\right) \Phi\left(x+z_{1}+z_{2}\right) \varpi\left(u_{1}-z_{1}, u_{2}-z_{2}\right) d^{2} z,
\end{aligned}
$$

where it is understood that $u_{3}=-u_{1}-u_{2}$ in the first integral.

LEMMA C.1. For any $z \in \mathbb{R}_{+}^{3}$, up to the permutation of $z_{1}$ and $z_{3}$, we have

$$
\begin{aligned}
& \sum_{\substack{v_{1}+\cdots+v_{4}=0 \\
v_{i} \in\{-1,1\}}} \sum_{|\mathbf{m}|=4} \mathbf{M}(\mathbf{m}) \tilde{G}^{\mathbf{m}}(v, z) \\
& =24\left(4\left[1-z_{2}\right]^{+}+2\left[1-z_{1}-z_{2}\right]^{+}\right. \\
& \quad+\frac{\left[2-z_{2}\right]^{+}}{2}+\frac{\left[2-z_{1}-z_{2}-z_{3}\right]^{+}}{2}+\left[2-z_{1}-z_{2}\right]^{+}-\left[2-z_{1}\right]^{+} \\
& \left.\quad-2 \max \left\{0,1-z_{1}, 2-z_{1}-z_{2}\right\}-2 \max \left\{0,1-z_{1}, 2-z_{1}-z_{2}-z_{3}\right\}\right) .
\end{aligned}
$$

PROOF. We fix $z \in \mathbb{R}_{+}^{3}$. We will proceed exactly as in the proof of Lemma 4.18 except that we will not give all the details. We will denote $\pm=+1$ or -1 and we let

$$
\begin{aligned}
\zeta_{1}= & \max ^{+}\left\{1-z_{1}, 2-z_{1}-z_{2}\right\}+\max \left\{1-z_{1}, 2-z_{1}-z_{2}-z_{3}\right\} \\
& +\underset{\max }{+}\left\{1-z_{1}-z_{2}, 2-z_{1}-z_{2}-z_{3}\right\}+2\left[2-z_{1}\right]^{+}+\left[2-z_{1}-z_{2}\right]^{+}, \\
\zeta_{2}= & 2\left(\left[1-z_{1}\right]^{+}+\left[1-z_{1}-z_{2}\right]^{+}+\left[1-z_{1}-z_{2}-z_{3}\right]^{+}\right), \\
\zeta_{3}= & \max _{+}^{+}\left\{1-z_{2}, 2-z_{2}-z_{3}\right\}+\left[2-z_{2}\right]^{+}+\left[1-z_{2}\right]^{+}+\left[1-z_{2}-z_{3}\right]^{+}, \\
\zeta_{4}= & 4\left[1-z_{1}\right]^{+}+2\left[1-z_{1}-z_{2}\right]^{+}, \\
\zeta_{5}= & 4\left[1-z_{2}\right]^{+}+2\left[1-z_{2}-z_{3}\right]^{+} .
\end{aligned}
$$

We will compute the values of $\tilde{\mathrm{G}}^{\mathbf{m}}(v, z)$ for all compositions $\mathbf{m}$ of 4 and all points $v=( \pm, \pm, \pm, \pm)$ such that $\sum v_{i}=0$. The computations are not difficult but there 
are many cases to check. By definition (1.26),

$$
\Lambda^{\mathbf{2 + 2}}(u)=\left(\begin{array}{cc}
0 & -u_{3}-u_{4} \\
u_{3}+u_{4} & 0
\end{array}\right) \equiv\left(\begin{array}{cc}
0 & -u_{1}-u_{2} \\
u_{1}+u_{2} & 0
\end{array}\right) .
$$

Then, by definition (1.28), we can check that

$$
\begin{aligned}
\tilde{\mathrm{G}}^{2+2}(++--) \equiv & \tilde{\mathrm{G}}^{\mathbf{2}+\mathbf{2}}(--++) \\
\equiv & 2\left(\left[2-z_{1}\right]^{+}+\left[2-z_{2}\right]^{+}+\left[2-z_{3}\right]^{+}\right. \\
& \left.+\left[2-z_{1}-z_{2}\right]^{+}+\left[2-z_{2}-z_{3}\right]^{+}+\left[2-z_{1}-z_{2}-z_{3}\right]^{+}\right) .
\end{aligned}
$$

We used that $v_{1}+v_{2}=0$ or 2 and that $z_{i} \geq 0$ to check which terms are a priori nonzero. Moreover, for similar reasons,

$$
\tilde{\mathrm{G}}^{2+2}(+-+-)=\tilde{\mathrm{G}}^{2+2}(+--+)=\tilde{\mathrm{G}}^{2+2}(-++-)=\tilde{\mathrm{G}}^{2+2}(-+-+)=0 .
$$

If we use the symmetry of the function $\Theta$ [see (4.73)], under the change of variable $z_{1} \leftrightarrow z_{3}$ and that $\mathrm{M}(\mathbf{2}+\mathbf{2})=-3$, we can conclude that

$$
\begin{aligned}
& \mathrm{M}(\mathbf{2}+\mathbf{2}) \sum_{\substack{v_{1}+\ldots+v_{4}=0 \\
v_{i} \in\{-1,1\}}} \tilde{\mathrm{G}}^{\mathbf{2 + 2}}(v, z) \\
& \quad \leftarrow-24\left(\left[2-z_{1}\right]^{+}+\frac{\left[2-z_{2}\right]^{+}}{2}+\left[2-z_{1}-z_{2}\right]^{+}+\frac{\left[2-z_{1}-z_{2}-z_{3}\right]^{+}}{2}\right)
\end{aligned}
$$

in the sense that if we replace the LHS of equation (C.6) by its RHS in formula (4.72), it does not change of the value of the integral. Let us continue with the compositions $\mathbf{3}+\mathbf{1}$ and $\mathbf{1}+\mathbf{3}$. We have

$$
\Lambda^{\mathbf{3}+\mathbf{1}}(u)=\left(\begin{array}{cc}
0 & -u_{4} \\
u_{4} & 0
\end{array}\right) \equiv\left(\begin{array}{cc}
0 & -u_{1} \\
u_{1} & 0
\end{array}\right)=-\Lambda^{\mathbf{1}+\mathbf{3}}(u) .
$$

This expression depends on a single variable (say $u_{1}$ ) and collecting the nonzero terms yields

$$
\begin{aligned}
\tilde{\mathrm{G}}^{\mathbf{3}+\mathbf{1}}( \pm, \pm, \pm, \pm) \equiv & \mathrm{G}^{\mathbf{1}+\mathbf{3}}( \pm, \pm, \pm, \pm) \\
\equiv & 2\left(\left[1-z_{1}\right]^{+}+\left[1-z_{2}\right]^{+}+\left[1-z_{3}\right]^{+}\right. \\
& \left.+\left[1-z_{1}-z_{2}\right]^{+}+\left[1-z_{2}-z_{3}\right]^{+}+\left[1-z_{1}-z_{2}-z_{3}\right]^{+}\right) .
\end{aligned}
$$

We can again use the symmetry of formula (4.72) and, since $\mathrm{M}(\mathbf{3}+\mathbf{1})=-2$, we get

$$
\begin{aligned}
\mathrm{M}(\mathbf{3}+\mathbf{1}) \sum_{\substack{v_{1}+\cdots+v_{4}=0 \\
v_{i} \in\{-1,1\}}} \tilde{\mathrm{G}}^{\mathbf{3}+\mathbf{1}}(v, z)+\tilde{\mathrm{G}}^{\mathbf{1}+\mathbf{3}}(v, z) \\
\leftarrow-48\left(2\left[1-z_{1}\right]^{+}+\left[1-z_{2}\right]^{+}\right. \\
\left.\quad+2\left[1-z_{1}-z_{2}\right]^{+}+\left[1-z_{1}-z_{2}-z_{3}\right]^{+}\right) \\
=-24\left(\zeta_{2}+2\left[1-z_{1}\right]^{+}+2\left[1-z_{2}\right]^{+}+2\left[1-z_{1}-z_{2}\right]^{+}\right) .
\end{aligned}
$$


Consider now the composition $\mathbf{1}^{4}=\mathbf{1}+\mathbf{1}+\mathbf{1}+\mathbf{1}$. By definition (1.26),

$$
\Lambda^{\mathbf{1}^{4}}=\left(\begin{array}{cccc}
0 & -u_{2} & -u_{2}-u_{3} & -u_{2}-u_{3}-u_{4} \\
u_{2} & 0 & -u_{3} & -u_{3}-u_{4} \\
u_{2}+u_{3} & u_{3} & 0 & -u_{4} \\
u_{2}+u_{3}+u_{4} & u_{3}+u_{4} & u_{4} & 0
\end{array}\right) .
$$

If we look at all permutations in $\mathbb{S}(4)$ and use the symmetry under permutations of the $u_{i}$ 's, we get

$$
\begin{aligned}
\tilde{G}^{1^{4}}(u, z) & \\
\equiv & 4\left(\underset{\max }{+}\left\{u_{1}-z_{1}, u_{1}+u_{2}-z_{1}-z_{2}, u_{1}+u_{2}+u_{3}-z_{1}-z_{2}-z_{3}\right\}\right. \\
& +\underset{+}{+}\left\{u_{1}-z_{1}-z_{2}-z_{3}, u_{1}+u_{2}-z_{1}-z_{2}, u_{1}+u_{2}+u_{3}-z_{1}\right\} \\
& +\max ^{+}\left\{u_{1}-z_{1}-z_{2}, u_{1}+u_{2}-z_{1}, u_{1}+u_{2}+u_{3}-z_{1}-z_{2}-z_{3}\right\} \\
& +\max ^{+}\left\{u_{1}-z_{1}-z_{2}-z_{3}, u_{1}+u_{2}-z_{1}, u_{1}+u_{2}+u_{3}-z_{1}-z_{2}\right\} \\
& +\underset{+}{+}\left\{u_{1}-z_{1}-z_{2}, u_{1}+u_{2}-z_{1}-z_{2}-z_{3}, u_{1}+u_{2}+u_{3}-z_{1}\right\} \\
& \left.+\max ^{+}\left\{u_{1}-z_{1}, u_{1}+u_{2}-z_{1}-z_{2}-z_{3}, u_{1}+u_{2}+u_{3}-z_{1}-z_{2}\right\}\right) .
\end{aligned}
$$

So we can assume that $\tilde{G}^{1^{4}}$ is given by the RHS of this expression, then it is straightforward to check that

$$
\begin{aligned}
& \tilde{G}^{\mathbf{1}^{4}}(++--)=8\left(\max ^{+}\left\{1-z_{1}, 2-z_{1}-z_{2}\right\}\right. \\
& \left.+\underset{\max }{+}\left\{1-z_{1}, 2-z_{1}-z_{2}-z_{3}\right\}+\left[2-z_{1}\right]^{+}\right), \\
& \tilde{G}^{\mathbf{1}^{4}}(-++-)=\tilde{G}^{\mathbf{1}^{4}}(+--+)=4 \zeta_{2}, \\
& \tilde{G}^{1^{4}}(+-+-)=4 \zeta_{4} \text {, } \\
& \tilde{G}^{1^{4}}(--++)=\tilde{G}^{1^{4}}(-+-+)=0
\end{aligned}
$$

so that, since $\mathrm{M}\left(\mathbf{1}^{4}\right)=-6$,

$$
\begin{aligned}
\mathrm{M}\left(\mathbf{1}^{4}\right) & \sum_{\substack{v_{1}+\ldots+v_{4}=0 \\
v_{i} \in\{-1,1\}}} \tilde{\mathrm{G}}^{1^{4}}(v, z) \\
= & -24\left\{2 \zeta_{2}+\zeta_{4}+2 \max \left\{1-z_{1}, 2-z_{1}-z_{2}\right\}\right. \\
& \left.+2 \stackrel{+}{\max }\left\{1-z_{1}, 2-z_{1}-z_{2}-z_{3}\right\}+2\left[2-z_{1}\right]^{+}\right\} .
\end{aligned}
$$


If we combine formulae (C.6), (C.7) and (C.8), we obtain

$$
\begin{aligned}
& \sum_{\substack{v_{1}+\cdots+v_{4}=0 \\
v_{i} \in\{-1,1\}}} \sum_{\substack{|\mathbf{m}|=4 \\
\ell(\mathbf{m}) \neq 3}} \mathrm{M}(\mathbf{m}) \tilde{\mathrm{G}}^{\mathbf{m}}(v, z) \\
& \quad \leftarrow-24\left\{3 \zeta_{2}+\zeta_{4}+2 \max \left\{1-z_{1}, 2-z_{1}-z_{2}\right\}\right.
\end{aligned}
$$

$$
\begin{aligned}
& +2 \underset{\max }{+}\left\{1-z_{1}, 2-z_{1}-z_{2}-z_{3}\right\}+2\left[1-z_{1}\right]^{+} \\
& +2\left[1-z_{2}\right]^{+}+2\left[1-z_{1}-z_{2}\right]^{+} \\
& \left.+3\left[2-z_{1}\right]^{+}+\frac{\left[2-z_{2}\right]^{+}}{2}+\left[2-z_{1}-z_{2}\right]^{+}+\frac{\left[2-z_{1}-z_{2}-z_{3}\right]^{+}}{2}\right\} .
\end{aligned}
$$

Finally, we look at the composition $\mathbf{2}+\mathbf{1}+\mathbf{1}$,

$$
\Lambda^{\mathbf{2}+\mathbf{1}+\mathbf{1}}(u)=\left(\begin{array}{ccc}
0 & -u_{3} & -u_{3}-u_{4} \\
u_{3} & 0 & -u_{4} \\
u_{3}+u_{4} & u_{4} & 0
\end{array}\right) \equiv\left(\begin{array}{ccc}
0 & -u_{1} & -u_{1}-u_{2} \\
u_{1} & 0 & -u_{2} \\
u_{1}+u_{2} & u_{2} & 0
\end{array}\right)
$$

and if we follow the same procedure, we can prove that

$$
\begin{aligned}
& \tilde{\mathrm{G}}^{\mathbf{2 + 1}+\mathbf{1}}(++--)=\mathrm{G}^{\mathbf{2 + 1}+\mathbf{1}}(--++)=\zeta_{1}+\zeta_{2}+\zeta_{3}, \\
& \tilde{\mathrm{G}}^{\mathbf{2 + 1}+\mathbf{1}}(+-+-)+\tilde{\mathrm{G}}^{\mathbf{2 + 1}+\mathbf{1}}(-+-+) \\
& \quad=\tilde{\mathrm{G}}^{\mathbf{2 + 1}+\mathbf{1}}(+--+)+\tilde{\mathrm{G}}^{\mathbf{2 + 1}+\mathbf{1}}(-++-)=2 \zeta_{2}+\zeta_{4}+\zeta_{5} .
\end{aligned}
$$

On the other hand,

$$
\begin{aligned}
\Lambda^{1+2+1} & =\left(\begin{array}{ccc}
0 & -u_{2}-u_{3} & -u_{2}-u_{3}-u_{4} \\
u_{2}+u_{3} & 0 & -u_{4} \\
u_{2}+u_{3}+u_{4} & u_{4} & 0
\end{array}\right) \\
& \equiv\left(\begin{array}{ccc}
0 & u_{1}+u_{2} & u_{2} \\
-u_{1}-u_{2} & 0 & -u_{1} \\
-u_{2} & u_{1} & 0
\end{array}\right) .
\end{aligned}
$$

It is not difficult to see that, up to conjugation by a permutation matrix, we have $\Lambda^{1+2+1} \equiv \Lambda^{2+1+1}$. This implies that $\tilde{\mathrm{G}}^{1+2+1} \equiv \tilde{\mathrm{G}}^{2+1+1}$ because such conjugation only changes the order of the sum over $\mathbb{S}(4)$ in the definition (1.28). Similarly, we can check that the matrix $\Lambda^{1+1+2}$ is also conjugated to $\Lambda^{2+1+1}$ by a permutation matrix, so that they give the same contribution to the 4 th cumulant. Since $\mathbf{M}(\mathbf{2}+\mathbf{1}+\mathbf{1})=4$, putting all terms together, we conclude that

$$
\sum_{\substack{v_{1}+\cdots+v_{4}=0 \\ v_{i} \in\{-1,1\}}} \sum_{\substack{\ell \mathbf{m} \mid=4 \\ \ell(\mathbf{m})=3}} \mathrm{M}(\mathbf{m}) \tilde{\mathrm{G}}^{\mathbf{m}}(v, z)=24\left\{\zeta_{1}+3 \zeta_{2}+\zeta_{3}+\zeta_{4}+\zeta_{5}\right\} .
$$


Observe that using the symmetry between $z_{1}$ and $z_{3}$ and the DHK formula (4.69), we can show that

$$
\begin{aligned}
\zeta_{1}+\zeta_{3} \leftarrow & 2\left(\left[1-z_{1}\right]^{+}+\left[1-z_{2}\right]^{+}+\left[1-z_{1}-z_{2}\right]^{+}+\left[2-z_{1}\right]^{+}\right. \\
& \left.+\frac{\left[2-z_{2}\right]^{+}}{2}+\left[2-z_{1}-z_{2}\right]^{+}+\frac{\left[2-z_{1}-z_{2}-z_{3}\right]^{+}}{2}\right),
\end{aligned}
$$

and we get

$$
\begin{aligned}
\sum_{\substack{v_{1}+\ldots+v_{4}=0 \\
v_{i} \in\{-1,1\}}} \sum_{\substack{|\mathbf{m}|=4 \\
\ell(\mathbf{m})=3}} \mathrm{M}(\mathbf{m}) \tilde{\mathrm{G}}^{\mathbf{m}}(v, z) \\
\leftarrow 24\left\{3 \zeta_{2}+\zeta_{4}+\zeta_{5}+2\left[1-z_{1}\right]^{+}+2\left[1-z_{2}\right]^{+}\right. \\
\quad+2\left[1-z_{1}-z_{2}\right]^{+}+2\left[2-z_{1}\right]^{+}+\left[2-z_{2}\right]^{+} \\
\left.\quad+2\left[2-z_{1}-z_{2}\right]^{+}+\left[2-z_{1}-z_{2}-z_{3}\right]^{+}\right\} .
\end{aligned}
$$

Finally, if we combine formulae (C.9) and (C.10), many terms cancel but not all of them and we are left with

$$
\begin{aligned}
\sum_{\substack{v_{1}+\ldots+v_{4}=0 \\
v_{i} \in\{-1,1\}}} \sum_{|\mathbf{m}|=4} \mathbf{M}(\mathbf{m}) \tilde{\mathbf{G}}^{\mathbf{m}}(v, z) \\
\leftarrow 24\left(\zeta_{5}-\left[2-z_{1}\right]^{+}+\frac{\left[2-z_{2}\right]^{+}}{2}+\frac{\left[2-z_{1}-z_{2}-z_{3}\right]^{+}}{2}\right. \\
\quad+\left[2-z_{1}-z_{2}\right]^{+}-2 \max ^{+}\left\{1-z_{1}, 2-z_{1}-z_{2}\right\} \\
\left.\quad-2 \max ^{+}\left\{1-z_{1}, 2-z_{1}-z_{2}-z_{3}\right\}\right) .
\end{aligned}
$$

Finally, if we make the change of variable $z_{1} \leftrightarrow z_{3}$,

$$
\zeta_{5} \leftarrow 4\left[1-z_{2}\right]^{+}+2\left[1-z_{1}-z_{2}\right]^{+},
$$

and we have proved the formula of Lemma C.1.

Acknowledgements. We thank Maurice Duits for helpful discussions regarding his related works on central limit theorems for orthogonal polynomial ensembles and Erik Duse for suggesting the proof of Proposition 1.9. We thank the referees for helpful comments and suggestions on the exposition.

\section{REFERENCES}

[1] Ameur, Y., Hedenmalm, H. and Makarov, N. (2011). Fluctuations of eigenvalues of random normal matrices. Duke Math. J. 159 31-81. MR2817648

[2] Amir, G., Corwin, I. and QuAstel, J. (2011). Probability distribution of the free energy of the continuum directed random polymer in $1+1$ dimensions. Comm. Pure Appl. Math. 64 466-537. MR2796514 
[3] Anderson, G. W., Guionnet, A. and Zeitouni, O. (2010). An Introduction to Random Matrices. Cambridge Studies in Advanced Mathematics 118. Cambridge Univ. Press, Cambridge. MR2760897

[4] Borodin, A. (2011). Determinantal point processes. In The Oxford Handbook of Random Matrix Theory 231-249. Oxford Univ. Press, Oxford. MR2932631

[5] Bourgade, P., Erdős, L., Yau, H.-T. and Yin, J. (2016). Fixed energy universality for generalized Wigner matrices. Comm. Pure Appl. Math. 69 1815-1881. MR3541852

[6] Boutet de Monvel, A. and Khorunzhy, A. (1999). Asymptotic distribution of smoothed eigenvalue density. I. Gaussian random matrices. Random Oper. Stoch. Equ. 7 1-22. MR1678012

[7] Boutet De Monvel, A. and Khorunzhy, A. (1999). Asymptotic distribution of smoothed eigenvalue density. II. Wigner random matrices. Random Oper. Stoch. Equ. 7 149-168. MR1689027

[8] BReuer, J. and Duits, M. (2016). Universality of mesoscopic fluctuations for orthogonal polynomial ensembles. Comm. Math. Phys. 342 491-531. MR3459158

[9] Breuer, J. and Duits, M. (2017). Central limit theorems for biorthogonal ensembles and asymptotics of recurrence coefficients. J. Amer. Math. Soc. 30 27-66. MR3556288

[10] Costin, O. and Lebowitz, J. L. (1995). Gaussian fluctuation in random matrices. Phys. Rev. Lett. 75 69-72. MR3155254

[11] Dean, D. S., Doussal, P. L., Majumdar, S. N. and Schehr, G. (2015). Finite temperature free fermions and the Kardar-Parisi-Zhang equation at finite time. Phys. Rev. Lett. 114110402.

[12] Deift, P., Kriecherbauer, T., Mclaughlin, K. T.-R., Venakides, S. and Zhou, X. (1999). Strong asymptotics of orthogonal polynomials with respect to exponential weights. Comm. Pure Appl. Math. 52 1491-1552. MR1711036

[13] Deift, P., Kriecherbauer, T., Mclaughlin, K. T.-R., Venakides, S. and Zhou, X. (1999). Uniform asymptotics for polynomials orthogonal with respect to varying exponential weights and applications to universality questions in random matrix theory. Comm. Pure Appl. Math. 52 1335-1425. MR1702716

[14] Duits, M. and Johansson, K. On mesoscopic equilibrium for linear statistics in Dyson's Brownian motion. Available at arXiv:1312.4295.

[15] Durrett, R. (2010). Probability: Theory and Examples, 4th ed. Cambridge Series in Statistical and Probabilistic Mathematics 31. Cambridge Univ. Press, Cambridge. MR2722836

[16] ERdős, L. and KNOwles, A. (2015). The Altshuler-Shklovskii formulas for random band matrices I: The unimodular case. Comm. Math. Phys. 333 1365-1416. MR3302637

[17] ERdôs, L. and KNOwLES, A. (2015). The Altshuler-Shklovskii formulas for random band matrices II: The general case. Ann. Henri Poincaré 16 709-799. MR3311888

[18] ERDŐs, L. and YAU, H.-T. (2012). Universality of local spectral statistics of random matrices. Bull. Amer. Math. Soc. (N.S.) 49 377-414. MR2917064

[19] Fyodorov, Y. V., Khoruzhenko, B. A. and Simm, N. J. (2016). Fractional Brownian motion with Hurst index $H=0$ and the Gaussian unitary ensemble. Ann. Probab. 44 2980-3031. MR3531684

[20] Gutzwiller, M. C. (1990). Chaos in Classical and Quantum Mechanics. Interdisciplinary Applied Mathematics 1. Springer, New York. MR1077246

[21] Hough, J. B., Krishnapur, M., Peres, Y. and Virág, B. (2006). Determinantal processes and independence. Probab. Surv. 3 206-229. MR2216966

[22] Johansson, K. (2006). Random matrices and determinantal processes. In Mathematical Statistical Physics 1-55. Elsevier, Amsterdam. MR2581882

[23] Johansson, K. (2007). From Gumbel to Tracy-Widom. Probab. Theory Related Fields 138 75-112. MR2288065 
[24] Kriecherbauer, T., Schubert, K., Schüler, K. and Venker, M. (2015). Global asymptotics for the Christoffel-Darboux kernel of random matrix theory. Markov Process. Related Fields 21 639-694. MR3494770

[25] Lambert, G. Mesoscopic fluctuations for unitary invariant ensembles. Preprint. Available at arXiv: 1510.03641 .

[26] LAmbert, G. CLT for biorthogonal ensembles and related combinatorial identities. Preprint. Available at arXiv:1511.06121.

[27] Le Doussal, P., Majumdar, S. N., Rosso, A. and Schehr, G. (2016). Exact short-time height distribution in 1D KPZ equation and edge fermions at high temperature. Phys. Rev. Lett. 117070403.

[28] Lieb, E. H. and Loss, M. (2001). Analysis, 2nd ed. Graduate Studies in Mathematics 14. Amer. Math. Soc., Providence, RI.

[29] Moshe, M., Neuberger, H. and Shapiro, B. (1994). Generalized ensemble of random matrices. Phys. Rev. Lett. 73 1497-1500. MR1291352

[30] Pastur, L. and Shcherbina, M. (2011). Eigenvalue Distribution of Large Random Matrices. Mathematical Surveys and Monographs 171. Amer. Math. Soc., Providence, RI. MR2808038

[31] Plancherel, M. and Rotach, W. (1929). Sur les valeurs asymptotiques des polynomes d'Hermite $H_{n}(x)=(-I)^{n} e^{\frac{x^{2}}{2}} \frac{d^{n}}{d x^{n}}\left(e^{-\frac{x^{2}}{2}}\right)$. Comment. Math. Helv. $1227-254$. MR1509395

[32] Rider, B. and ViRÁG, B. (2007). Complex determinantal processes and $H^{1}$ noise. Electron. J. Probab. 12 1238-1257. MR2346510

[33] RIDER, B. and VIRÁG, B. (2007). The noise in the circular law and the Gaussian free field. Int. Math. Res. Not. IMRN 2 Art. ID rnm006, 33. MR2361453

[34] Sas Amoto, T. and Spohn, H. (2010). The crossover regime for the weakly asymmetric simple exclusion process. J. Stat. Phys. 140 209-231. MR2659278

[35] Sasamoto, T. and Spohn, H. (2010). Exact height distributions for the KPZ equation with narrow wedge initial condition. Nuclear Phys. B 834 523-542. MR2628936

[36] Simon, B. (2005). Orthogonal Polynomials on the Unit Circle. Part 1: Classical Theory. American Mathematical Society Colloquium Publications 54. Amer. Math. Soc., Providence, RI. MR2105088

[37] Simon, B. (2005). Trace Ideals and Their Applications, 2nd ed. Mathematical Surveys and Monographs 120. Amer. Math. Soc., Providence, RI. MR2154153

[38] Soshnikov, A. (2000). Determinantal random point fields. Russian Math. Surveys 55923 975.

[39] Soshnikov, A. (2000). The central limit theorem for local linear statistics in classical compact groups and related combinatorial identities. Ann. Probab. 28 1353-1370. MR1797877

[40] Soshnikov, A. (2002). Gaussian limit for determinantal random point fields. Ann. Probab. 30 171-187. MR1894104

DEPARTMENT OF MATHEMATiCS

KTH ROYAL INSTITUTE OF TECHNOLOGY

LINDSTEDTSVÄGEN 25

10044 STOCKHOLM

SWEDEN

E-MAIL: kurtj@kth.se
INSTITUT FÜR MATHEMATIK UNIVERSITY OF ZÜRICH WINTERTHURERSTRASSE 190 CH-8057 ZÜRICH SWITZERLAND

E-MAIL: glambert@kth.se 